\title{
Orders on partial partitions based on block apportioning
}

\author{
Christian Ronse
}

Received: date / Revised: date

\begin{abstract}
Some problems in image analysis require considering an order on space partitions: in image filtering the partition of image zones must grow, while in image segmentation one maximizes the partition into image objects.

In the classical refinement order, the growth of a partition is achieved through the merging of blocks. We generalize this operation into block apportioning: some blocks in a partial partition may be split, and their parts are merged with some remaining blocks; this includes the possibility of a block being merged with another without being split. We study this operation in detail; we obtain then the apportioning order, which extends the refinement order.

For partial partitions, the standard order combines the merging of blocks with the growth of existing blocks and the creation of new blocks. Combining these operations with block apportioning leads to the apportioning-inflating and extended orders; the latter contains the standard order. Our analysis rests on a study of Serra's building order on partial partitions, which intervenes in the above new orders.

Except the building order, all these orders are graded, with a compound grading for the apportioning-inflating and extended orders; this gives the number of elementary operations involved in a growth of a partial partition.
\end{abstract}

Keywords partial partition · partial order $\cdot$ block apportioning $\cdot$ image analysis

Mathematics Subject Classification (2010) 03E02 · 06A99 · 68U10

\section{Introduction}

This paper is a sequel to [16], which studied some new orders on partial partitions; it also elaborates on some parts of [15].

This work received funding from the Agence Nationale de la Recherche, contract ANR-2010BLAN-0205-01.

Christian Ronse

ICube, Université de Strasbourg, CNRS, 300 Boulevard Sébastien Brant, CS 10413, 67412 ILLKIRCH CEDEX, FRANCE — E-mail: cronse@unistra.fr, URL: http://icube-miv.unistra.fr/ 
Given a set $E$, a partition of $E$ is a family of non-void mutually disjoint subsets of $E$, called blocks [11], whose union is $E$. A partial partition of $E$ is a family of non-void mutually disjoint blocks, but their union is not necessarily equal to $E$; the union of all blocks of a partial partition $\pi$ is the support of $\pi, \operatorname{supp}(\pi)$; thus $\pi$ is a partition of $\operatorname{supp}(\pi)$; let the background of $\pi$ be the complement of the support, $\operatorname{back}(\pi)=E \backslash \operatorname{supp}(\pi)$. We write $\Pi(E)$ for the set of all partitions of $E$, and $\Pi^{*}(E)$ for the set of all partial partitions of $E$.

The customary order on $\Pi(E)$ is refinement [11]: for $\pi_{1}, \pi_{2} \in \Pi(E)$, we say that $\pi_{1}$ is finer than $\pi_{2}$, or that $\pi_{2}$ is coarser than $\pi_{1}$, and write $\pi_{1} \leq \pi_{2}$ (or $\pi_{2} \geq \pi_{1}$ ), if and only if every block of $\pi_{1}$ is included in a block of $\pi_{2}$, equivalently, every block of $\pi_{2}$ is a union of blocks of $\pi_{1}$. Then $\leq$ is a partial order relation on $\Pi(E)$, for which $\Pi(E)$ is a complete lattice, whose least (finest) and greatest (coarsest) elements are the identity partition $\mathbf{0}_{E}$ (whose blocks are all singletons in $E$ ) and the universal partition $\mathbf{1}_{E}$ (with $E$ as single block) [11]. See [14] for more details.

Partial partitions of a set $E$ were first studied by Draškovičová $[4,5]$, who called them "partitions in $E$ ". She ordered $\Pi^{*}(E)$ similarly to the refinement order: for $\pi_{1}, \pi_{2} \in \Pi^{*}(E)$, we write $\pi_{1} \leq \pi_{2}$ (or $\pi_{2} \geq \pi_{1}$ ), if and only if every block of $\pi_{1}$ is included in a block of $\pi_{2}$. This order $\leq$ constitutes $\Pi^{*}(E)$ into a complete lattice whose least and greatest elements are the empty partial partition $\emptyset$ and the universal partition $\mathbf{1}_{E}$; it was further studied in $[13,14,16]$. Note however that for $\pi_{1}, \pi_{2} \in \Pi^{*}(E)$ with $\pi_{1} \leq \pi_{2}$, a block of $\pi_{2}$ will not necessarily be a union of blocks of $\pi_{1}$ (as it was the case with partitions): blocks of $\pi_{2}$ can be constructed by merging several blocks of $\pi_{1}$, inflating individual blocks of $\pi_{1}$, or both combined, or else by creating new blocks outside $\operatorname{supp}\left(\pi_{1}\right)$. Hence $[15,16]$ we do not call this order on $\Pi^{*}(E)$ refinement, but the standard order. In fact the refinement order is the restriction to partitions of the standard order on partial partitions.

Partitions and the refinement order are relevant to image segmentation. A grey-level or colour image can be represented as a function $F: E \rightarrow T$, where $E$ is the space of pixels and $T$ is the set of image values (grey-levels or colours). A segmentation of the image $F$ is a partition of $E$ into visually significant regions representing objects or the surrounding background. As explained in $[15,16]$, the conventional requirements for image segmentation outlined in [25] mean that the segmentation process selects, among all partitions with connected blocks on which the image is homogeneous, one that is coarsest possible. More precisely:

1. To each image $F$ on $E$, the segmentation method associates the family $\mathcal{C}^{F}$ of all subsets of $E$ that are "connected" (according to a choice of connectivity) and on which the image $F$ is "homogeneous" (according to some criterion).

2. Let $\Pi\left(E, \mathcal{C}^{F}\right)=\Pi(E) \cap \mathcal{P}\left(\mathcal{C}^{F} \backslash\{\emptyset\}\right)$ be the set of all partitions of $E$ whose blocks belong to $\mathcal{C}^{F} \backslash\{\emptyset\}$. Then the segmentation of $F$ must be a maximal element, for the refinement order, of $\Pi\left(E, \mathcal{C}^{F}\right)$.

Serra $[21,17]$ introduced the paradigm of connective segmentation. Here the set $\mathcal{C}^{F}$ must be a connection, i.e., $\mathcal{C}^{F}$ comprises all singletons, and a union of overlapping elements of $\mathcal{C}^{F}$ will belong to $\mathcal{C}^{F}$; equivalently, $\mathbf{0}_{E} \in \Pi\left(E, \mathcal{C}^{F}\right)$ and $\Pi\left(E, \mathcal{C}^{F}\right)$ must be closed under the supremum operation of the complete lattice $\Pi(E)$. Then the segmentation of $F$ will be the greatest element of $\Pi\left(E, \mathcal{C}^{F}\right)$. A well-known examples is when $\mathcal{C}^{F}$ consists of all connected subsets of $E$ on which $F$ is constant, and then the segmentation will be the partition of all flat zones of $F$, i.e., maximal connected subsets of $E$ on which $F$ has a constant value. 


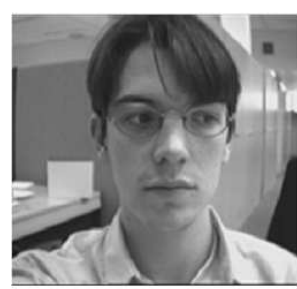

a)

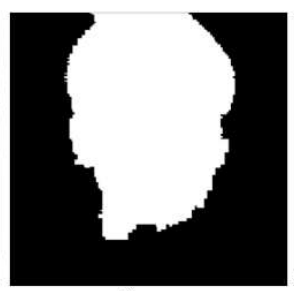

b)

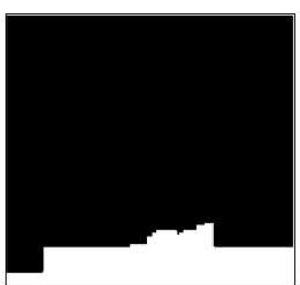

c)

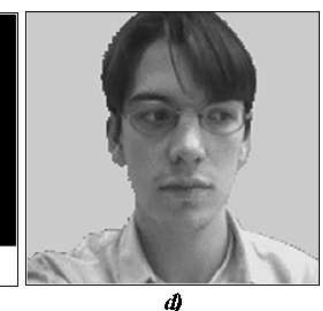

d)

Fig. 1 (From [17], following [6].) (a) Initial image of the silhouette to be segmented. (b) Segmentation of the face (by a colour criterion). (c) Marker for the bust. (d) Final segmentation.

Connective segmentation underlies the connected filtering of images $[21,19,17$, 18,24]. To every image $F: E \rightarrow T$ corresponds a partition $\pi(F)$ of $E$ obtained by a connective segmentation. Given an operator $\psi: T^{E} \rightarrow T^{E}$ for transforming images, we say that $\psi$ is connected if it coarsens the segmentation partition corresponding to the image: $\pi(F) \leq \pi(\psi(F))$. Initially, this notion was considered for the segmentation into flat zones, then $\psi$ is connected if it coarsens the partition into flat zones; since visible edges (in particular object contours) lie at the frontier between distinct flat zones, it follows that a connected operator can either preserve or eliminate such edges, but never displace or deform them.

Working exclusively with partitions is limiting, since the known operations have been mostly merging and splitting blocks, in other words coarsening and refining a partition, which were used in alternation in the split-and-merge approaches to image segmentation [12]. The use of partial partitions in image segmentation was justified in $[13,16]$. Let us just mention two facts:

- Segmentations are often built by iteratively growing partial partitions; for example: in region growing, blocks are inflated from seeds; now compound segmentation $[20,17,15]$ proceeds by constructing an initial partial partition according to a first criterion, then a partial partition of the background according to a second criterion, and the final segmentation is the union of the two, see for example Figure 1.

- Some segmentation algorithms produce a partial partition, whose background consists of frontiers separating the blocks; this frontier can be thick, see for example Figure 2. Some segmentation algorithms can miss whole regions that remain thus in the background.

In $[13,17]$, the framework of connective segmentation was extended to partial partitions. Let $\Pi^{*}\left(E, \mathcal{C}^{F}\right)=\Pi^{*}(E) \cap \mathcal{P}\left(\mathcal{C}^{F} \backslash\{\emptyset\}\right)$ be the set of all partial partitions of $E$ whose blocks belong to $\mathcal{C}^{F} \backslash\{\emptyset\}$. Here $\mathcal{C}^{F}$ must be a partial connection, i.e., a union of overlapping elements of $\mathcal{C}^{F}$ will belong to $\mathcal{C}^{F}$; equivalently, $\emptyset \in \Pi \Pi^{*}\left(E, \mathcal{C}^{F}\right)$ and $\Pi^{*}\left(E, \mathcal{C}^{F}\right)$ must be closed under the supremum operation of the complete lattice $\Pi^{*}(E)$. Then the segmentation of $F$ will be the greatest element of $\Pi^{*}\left(E, \mathcal{C}^{F}\right)$.

An example is given by the regional Lipschitz segmentation [21] (also called smooth segmentation [17]). It depends on two numerical parameters, a radius $r>0$ and a slope $s>0$, and has two versions, "by erosion" and "by opening". Given a function $F: E \rightarrow T$, let $L E_{F}^{r, s}$ be the set of all $p \in E$ such that the restriction of $F$ to $B_{r}(p)$, the ball of radius $r$ centered about $p$, satisfies the Lipschitz condition 

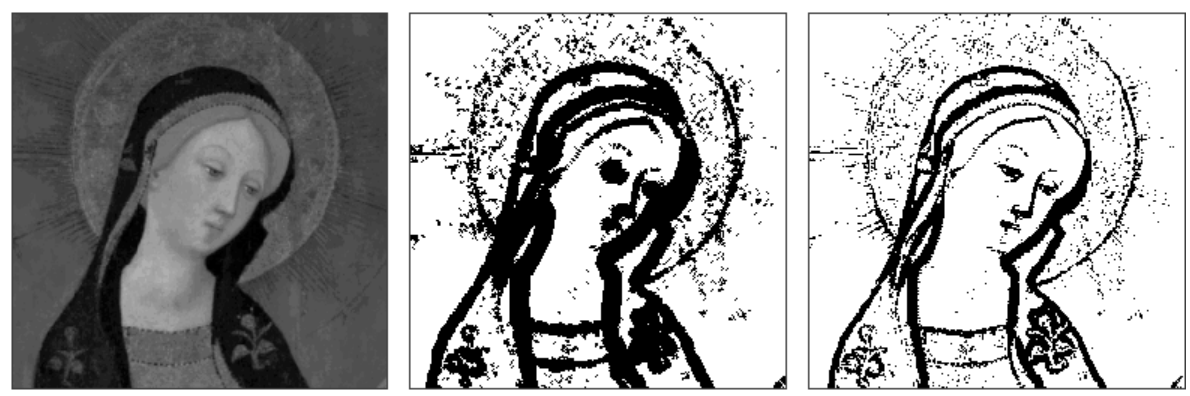

Fig. 2 (From [21].) Left: original grey-level image. Middle and right: regional Lipschitz segmentation, respectively "by erosion" and "by opening", with radius $r=7$ and slope $s=6$; the blocks are the white connected components, they correspond to regions, and the background is in black, it represents the frontiers.

of slope $s$; let $L O_{F}^{r, s}$ be the union of all balls $B_{r}(p)$ for $p \in L E_{F}^{r, s}$, in other words the dilation of $L E_{F}^{r, s}$ by the ball $B_{r}$. The segmentation "by erosion" (resp., "by opening") consists of the partial partition of connected components of $L E_{F}^{r, s}$ (resp., $\left.L O_{F}^{r, s}\right)$. We illustrate both versions of this segmentation in Figure 2, where we see that they produce thick frontiers between regions.

When a segmentation produces a partial partition with a background made of thick frontiers, the latter can be thinned by applying a region growing algorithm to the blocks, this time relying on another criterion than the one used in the segmentation. Region growing, whether in the segmentation algorithm or in the post-segmentation processing, follows a partial order relation on $\Pi^{*}(E)$ :

- The inflating order $\stackrel{i}{\leq}$ (written $\unlhd$ in $[15,16])$ : for $\pi_{1}, \pi_{2} \in \Pi^{*}(E), \pi_{1} \stackrel{i}{\leq} \pi_{2}$ iff every block of $\pi_{1}$ is included in a (unique) block of $\pi_{2}$, and every block of $\pi_{2}$ contains exactly one block of $\pi_{1}$; in other words $\pi_{2}$ is obtained from $\pi_{1}$ by inflating its blocks.

On the other hand, the compound segmentation paradigm [20,17,15] follows another order on $\Pi^{*}(E)$ :

- The inclusion order $\subseteq$ : for $\pi_{1}, \pi_{2} \in \Pi^{*}(E), \pi_{1} \subseteq \pi_{2}$ simply means that each block of $\pi_{1}$ is a block of $\pi_{2}$, in other words $\pi_{2}$ is obtained from $\pi_{1}$ by adding new blocks to it.

A third order on $\Pi^{*}(E)$ is involved in the merging of blocks:

- The merging order $\stackrel{\mathrm{m}}{\leq}$ (written $\sqsubseteq$ in $[15,16]$ and called pure refinement order in [15]): for $\pi_{1}, \pi_{2} \in \Pi^{*}(E), \pi_{1} \stackrel{\mathrm{m}}{\leq} \pi_{2}$ iff $\pi_{1}$ and $\pi_{2}$ share the same support and $\pi_{1} \leq \pi_{2}$ according to the refinement order for partitions of that support; equivalently, every block of $\pi_{1}$ is included in a (unique) block of $\pi_{2}$, and every block of $\pi_{2}$ is a union of blocks of $\pi_{1}$.

These 3 orders are the basic orders on $\Pi^{*}(E)$ [16], they are included in the standard order $\leq$. Combining any two of them, one generates 3 compound orders:

- Combining inclusion $\subseteq$ and inflating $\stackrel{i}{\leq}$, one gets the inclusion-inflating order $\stackrel{i}{\subseteq}($ written $\varrho$ in $[15,16])$ : for $\pi_{1}, \pi_{2} \in \Pi^{*}(E), \pi_{1} \stackrel{i}{\subseteq} \pi_{2}$ iff every block of $\pi_{1}$ is 


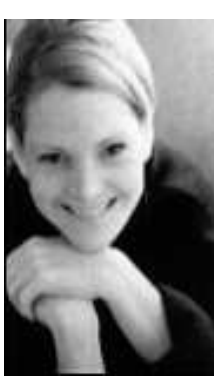

(a)

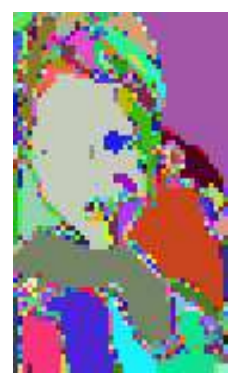

(b)

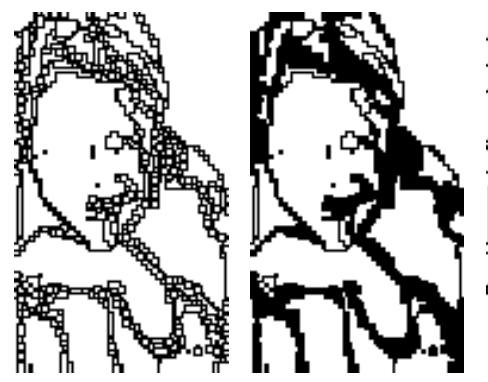

(c) (d)

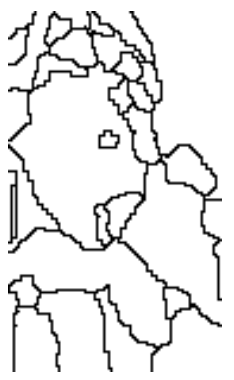

(e)

Fig. 3 From [10]: (a) Original image. (b,c) Segmentation: we show (b) the blocks in pseudocolour, and (c) the contours of the blocks. Following [22,23]: (d) The blocks of size less than 20 pixels are removed (they are shown in black), and (e) the remaining blocks of size $>20$ are inflated to cover the removed ones, we show the contours of the blocks.

included in a (unique) block of $\pi_{2}$ and every block of $\pi_{2}$ contains at most one block of $\pi_{1}$; equivalently, $\pi_{2}$ is obtained from $\pi_{1}$ by inflating existing blocks and creating new blocks.

- Combining merging $\stackrel{m}{\leq}$ and inflating $\stackrel{i}{\leq}$, one gets the merging-inflating order $\stackrel{\mathrm{mi}}{\leq}$ (written $\unrhd$ in $[15,16]$ and called refinement-inflating order in [15]): for $\pi_{1}, \pi_{2} \in$ $\Pi^{*}(E), \pi_{1} \stackrel{\text { mi }}{\leq} \pi_{2}$ iff every block of $\pi_{1}$ is included in a (unique) block of $\pi_{2}$ and every block of $\pi_{2}$ contains at least one block of $\pi_{1}$; equivalently, $\pi_{2}$ is obtained from $\pi_{1}$ by inflating and merging its blocks.

Finally combining inclusion $\subseteq$ and merging $\stackrel{m}{\leq}$, one obtains the standard order $\leq$, which contains the 5 others. The 3 basic and 3 compound orders were studied in [16], where we gave examples of their possible applications in image analysis and segmentation.

Beside thick frontiers separating regions, or incomplete segmentations, there is another problem in image segmentation, which was addressed by Serra $[22,23]$. Many image segmentation algorithms will produce, on a gradual transition between two zones, small "parasitic" blocks in the partial partition, See Figure 3 (b,c). Serra proposed to first remove "parasitic" blocks, next to inflate the remaining blocks until the removed blocks are completely covered (for instance, one can take the Voronoi diagram), see Figure 3 (d,e).

Now we consider this compound process, with two successive steps, as a single operation that we call apportioning. Some blocks are removed, and their contents is apportioned between one or several remaining blocks. In other words, a disappearing block either is merged with one remaining block, or it is split into several pieces, and each piece is merged with some remaining block. Block merging is thus a special case of apportioning, when the disappearing blocks are not split. See Figure 4. This operation leads to a new partial order on partitions, which we call the apportioning order and write $\stackrel{\text { a }}{\leq}$; it contains the merging order: $\forall \pi_{1}, \pi_{2} \in \Pi^{*}(E)$, $\pi_{1} \stackrel{\mathrm{m}}{\leq} \pi_{2} \Rightarrow \pi_{1} \stackrel{\mathrm{a}}{\leq} \pi_{2}$. This order was briefly suggested in Serra's work [22,23], but was not pursued further. 

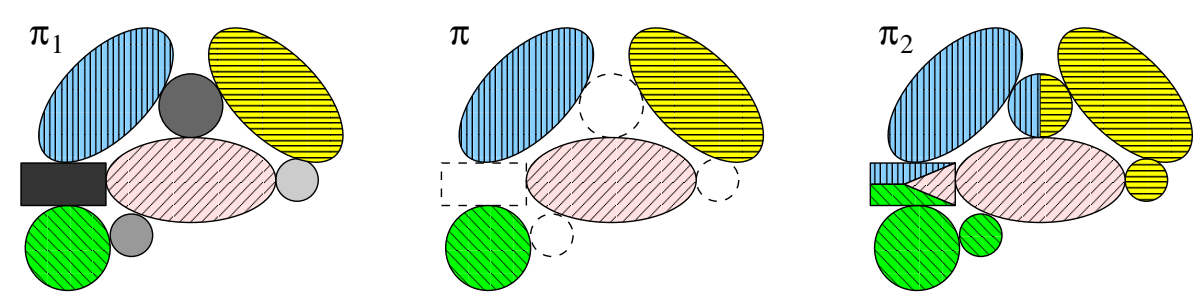

Fig. 4 Apportioning. Left: $\pi_{1}$, the blocks to be removed are in grey, and the preserved ones are in colour with a black hatching. Middle: partial partition $\pi$ with blocks removed. Right: one removed block is split into three parts, another one into two parts, and two remain in one part; then each part is merged with a preserved block, resulting in $\pi_{2}$. The removed blocks of $\pi_{1}$ have been apportioned. We have $\pi_{1} \supseteq \pi^{\leq} \leq \pi_{2}$ and $\operatorname{supp}\left(\pi_{1}\right)=\operatorname{supp}\left(\pi_{2}\right)$. We write $\pi_{1} \stackrel{\text { a }}{\leq} \pi_{2}$.

This problem with "parasitic" zones on gradual transitions between objects, and the solution provided by apportioning, arise also in image filtering. Suppose that one wants to sharpen the edge between two homogeneous zones separated by a gradual transition. We show in Figure 5 (a) the grey-level profile across the transition. By connected filtering, we can merge flat zones; for instance in (b,c) all singleton zones have been merged with a neighbouring non-singleton zone, getting the latter's grey-level; in $(\mathrm{d}, \mathrm{e})$ the merging goes further, all small flat zones have been merged with a large flat zone. However the resulting edge will not be located at the middle of the original transition, since the latter lies inside a flat zone. In (f) we show a sharpening where the edge lies at the middle of the transition; it is obtained by eliminating all small zones, then inflating equally the two large zones (through a Voronoi diagram); in other words, small flat zones have been apportioned to the large ones, using the method proposed by Serra. Such an edge sharpening that breaks image zones cannot be achieved by connected filtering; it is similar to the behaviour of shock filters (see [26] for a recent survey and extension).

An alternative view of this type of filtering, where transition zones can be eliminated, is that we associate to an image $F$ defined on $E$ a partial partition $\pi^{*}(F)$ of zones corresponding to significant homogeneous regions, excluding those corresponding to noise or transitions. Then an image processing operator $\psi$ can be characterized in terms of its behaviour on $\pi^{*}(F)$. For instance, if in Figure 5 (a) we discard the singleton zones, working on the partial partition of non-singleton flat zones, we can obtain the results (b,c,d,e); now if we discard all small flat zones and work on the partial partition of large flat zones, we can obtain the result (f).

Apportioning intervenes implicitly in some region growing methods such as watershed segmentation. Algorithms for constructing an image watershed often face the situation where a crest line emerges through a narrow passage into a wide plateau surrounded by higher crests, see for instance the left part of Figure 6 (from of [1]); for some algorithms, the plateau and the surrounding crests will be incorporated into the watershed (it becomes thus a "thick boundary"), while for others the plateau will be discarded, belonging neither to a basin nor to the watershed; for a few algorithms, this plateau will be included into one basin. Now the algorithm in [1] nicely apportions this plateau between the watershed line and the two basins separated by it, as seen in Figure 6, right. 

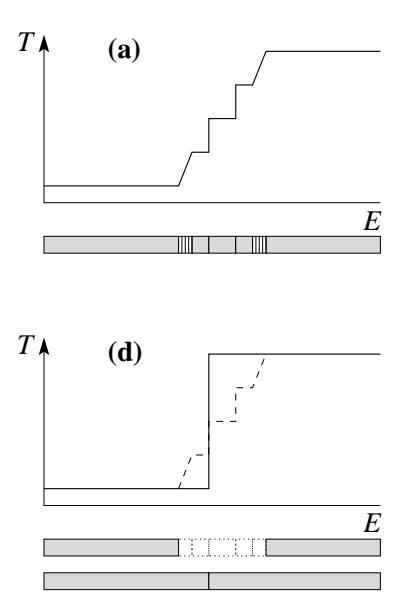
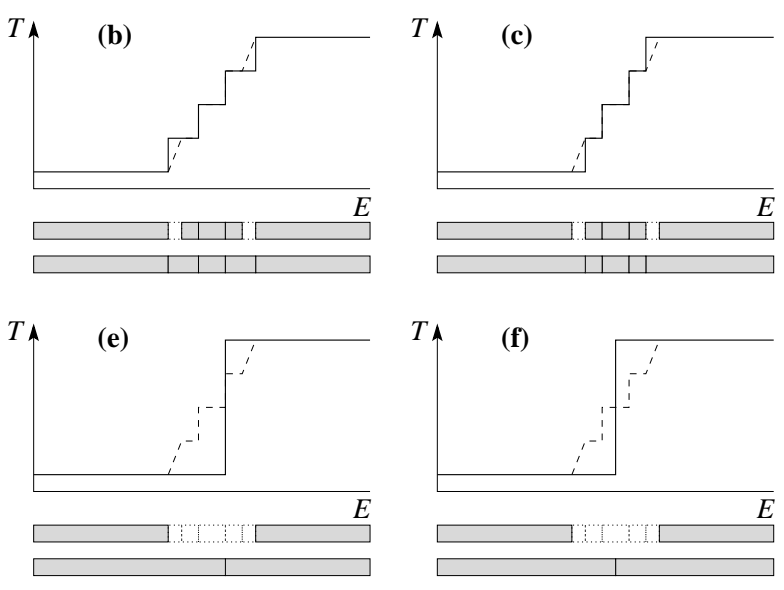

Fig. 5 (a) The graph of a one-dimensional signal across a gradual step edge; below we show its segmentation into flat zones, with light grey rectangles for non-singleton zones, and vertically hatched ones for groups of singleton zones. (b,c) The edge is partially sharpened by merging singleton zones with a neighbouring non-singleton zone. (d,e) We can merge both singleton and small non-singleton zones with the closest large zone, but for the middle zone there are two choices. (f) The edge is sharpened by eliminating all small zones, then inflating equally the two large ones; the edge between them lies inside the previous middle zone.
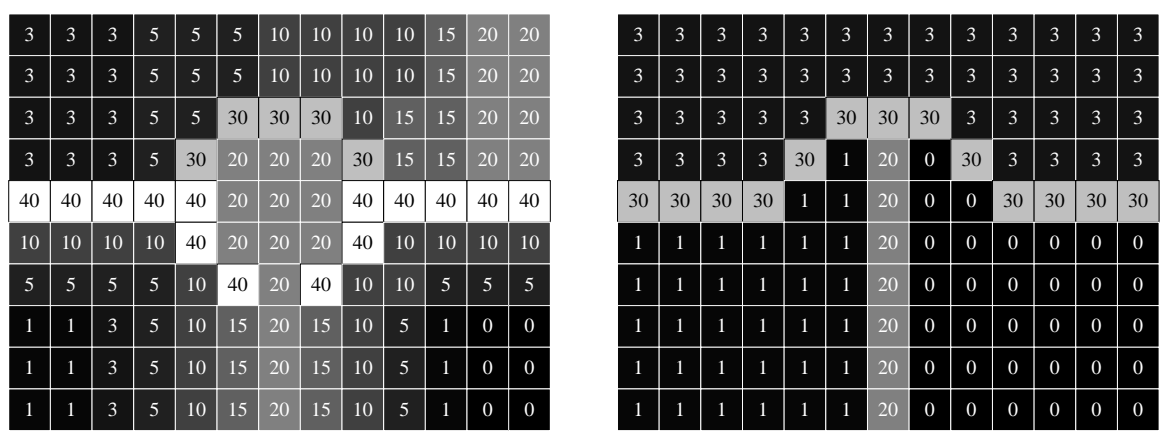

Fig. 6 (From [1].) Each square represents a pixel, with the number inside giving its greylevel. Left: two regional minima originating from the bottom left and right corners have their catchment basins meeting at a crest line of level 20 (bottom middle), and the latter prolongs itself into a square plateau (center) surrounded by crests with levels 30 and 40. Right: in the segmentation, the plateau is apportioned between the two basins and the watershed line.

Instead of the apportioning order, Serra [22,23] studied a more general partial order on $\Pi^{*}(E)$ that he called the building order. He wrote it $\preceq$, but we write it $\Subset$, since we use the symbol $\prec$ for covering relations $[15,16]$. For $\pi_{1}, \pi_{2} \in \Pi^{*}(E)$, $\pi_{1} \Subset \pi_{2}$ if and only if every block of $\pi_{2}$ contains at least one block of $\pi_{1}$. Then the two successive operations of removing some blocks and then inflating remaining blocks, are both growing for the order $\Subset: \pi_{1} \supseteq \pi \stackrel{i}{\leq} \pi_{2} \Rightarrow \pi_{1} \Subset \pi \Subset \pi_{2}$. Conversely, we will see that when $\pi_{1} \Subset \pi_{2}$, there exists $\pi \in \Pi^{*}(E)$ such that we obtain $\pi$ by removing some blocks of $\pi_{1}$, then $\pi_{2}$ by inflating blocks of $\pi: \pi_{1} \supseteq \pi \stackrel{i}{\leq} \pi_{2}$. 
Now the apportioning order is the restriction of the building order to partial partitions sharing the same support: for $\pi_{1}, \pi_{2} \in \Pi^{*}(E), \pi_{1} \stackrel{a}{\leq} \pi_{2}$ if and only if $\pi_{1} \Subset \pi_{2}$ and $\operatorname{supp}\left(\pi_{1}\right)=\operatorname{supp}\left(\pi_{2}\right)$. Following the generalization of merging into apportioning, we will generalize the merging-inflating and standard orders into the following two orders:

- The apportioning-inflating order $\stackrel{\text { ai }}{\leq}$, where $\pi_{1} \stackrel{\text { ai }}{\leq} \pi_{2}$ if $\pi_{2}$ is obtained from $\pi_{1}$ by apportioning and inflating its blocks, see Figure 11 ; equivalently, $\pi_{1} \Subset \pi_{2}$ and $\operatorname{supp}\left(\pi_{1}\right) \subseteq \operatorname{supp}\left(\pi_{2}\right)$.

- The extended order $\stackrel{\mathrm{e}}{\leq}$, where $\pi_{1} \stackrel{\mathrm{e}}{\leq} \pi_{2}$ if $\pi_{2}$ is obtained from $\pi_{1}$ by creating then apportioning blocks, see Figure 13 ; equivalently, $\pi_{1} \Subset \pi_{2} \cap \mathcal{P}\left(\operatorname{supp}\left(\pi_{1}\right)\right)$ and $\operatorname{supp}\left(\pi_{1}\right) \subseteq \operatorname{supp}\left(\pi_{2}\right)$.

We saw above that thick boundaries between regions can be thinned by inflating blocks of the partial partition, while small "parasitic" segmentation classes can be eliminated by apportioning blocks. Thus when both problems arise together, the solution will combine inflating and apportioning blocks, thus the partial partition will grow according to the apportioning-inflating order.

As explained before, algorithms for image segmentation often construct the partial partition by inflating and creating blocks from initial seeds. Thus if we consider the compound process of first creating the segmentation, then improving it, we will combine inflating, creating and apportioning blocks, thus the partial partition will grow according to the extended order. Block apportioning could also be applied in the initial construction and growth of the segmentation, for instance if a region ceases to satisfy a criterion; this idea is implicitly contained in the split-and-merge strategy for segmentation [12].

Image segmentation has been integrated into the framework of hierarchies, where coarser segmentation partitions are built by merging some segmentation classes from finer ones [8]; in the case of watersheds, the merging can be guided by the saliency of watershed lines [9]. Combining this with the growth of zones, the merging-inflating order underlies such a hierarchical segmentation. Note also that one can also remove markers, and the unmarked basins will no more grow into regions, but will be apportioned between neighbouring regions. Thus the apportioning-inflating order could be used for modeling the growth and evolution of regions in hierarchical segmentation.

This paper gives a detailed mathematical study of these 3 new orders on partial partitions based on the operation of apportioning blocks. The apportioning order is basic (as the inflating, inclusion and merging orders), while the apportioninginflating and extended orders are compound (as the inclusion-inflating, merginginflating and standard orders). In fact, the extension of the merging, merginginflating and standard orders into the apportioning, apportioning-inflating and extended orders respectively translates several properties of the former into similar properties of the latter.

A significant aspect of orders on $\Pi^{*}(E)$ is the Jordan-Dedekind chain condition, namely that all maximal chains in an interval have the same length. This is important in practice (when $E$ is finite), since it means that when one constructs a partial partition by a succession of elementary growth operations, the number of steps always remains constant, it will be equal to the height of that partial partition. Our 3 new orders satisfy that property (as do the 6 orders studied in [16]). 
In fact, the apportioning-inflating and extended orders, being compound, use two elementary growth operations, and the height function is the sum of two functions counting the number of times that each operation is performed.

On the other hand the building order does not satisfy the chain condition, and its maximal chains are counter-intuitive. Probably this is linked to the fact that the growth in this order involves two operations, inflating and removing blocks, one increasing the support and the other decreasing it. In our opinion, the building order is not specific enough to be meaningful in practice.

The 6 (3 basic and 3 compound) orders on $\Pi^{*}(E)$ studied in [16], the 3 new ones introduced here and the identity, are ordered by inclusion and constitute a lattice whose Hasse diagram is illustrated in Figure 14. Note that the standard order is the only one among the 10 that forms a lattice on $\Pi^{*}(E)$; however the merging order is the disjoint union of the lattices $\Pi(A), A \in \mathcal{P}(E)$, ordered by refinement.

\section{Paper organization}

Section 2 gives our notation and summarizes the main concepts and results of [16], about multiple grading and height functions, then the merging, inflating and inclusion orders and their combinations. Section 3 studies the apportioning order and its combinations with basic orders: the apportioning-inflating and extended orders. Section 4 concludes, summarizing our results, discussing their relevance and putting them into perspective. The Appendix analyses the "bad" properties of the building order.

\section{Mathematical preliminaries}

Subsection 2.1 recalls general notions about orders, covering relations, maximal chains, grading and height functions, then the multiple grading introduced in [16]. Subsection 2.2 summarizes the mathematical definition and main properties of the 6 orders studied in [16].

But we first give some general notation. In mathematical formulas, we will write "\&" for the logical "and". For a set $A$, we will write $\mathcal{P}(A)$ for the set of parts of $A$, and $|A|$ for the cardinal of $A$. Given two subsets $A$ and $B$ of a set $E$, we say that $A$ and $B$ overlap, and write $A \varnothing B$, if $A \cap B \neq \emptyset$.

Every binary relation $R$ is identified with the set of ordered pairs $(a, b)$ such that $a R b$; thus the inclusion $R \subseteq S$ means that $(a R b) \Rightarrow(a S b)$, and the union $R \cup S$ is given by $a(R \cup S) b \Leftrightarrow(a R b)$ or $(a S b)$. The composition $R \cdot S$ of two relations is defined by $a R \cdot S b \Leftrightarrow \exists c,(a R c) \&(c S b)$. In particular, we will express each compound order on $\Pi^{*}(E)$ as a composition of two basic orders.

\subsection{Orders, grading and height}

Our terminology on orders and lattices follows [2,3,7]. See also [16]. We will consider several distinct (partial) order relations, they will be identified by a superscript on the usual order symbol, say $\stackrel{x}{\leq}$ for "less than or equal to", $\stackrel{x}{<}$ for "strictly 
less than", $\stackrel{x}{\geq}$ for "greater than or equal to", $\stackrel{x}{>}$ for "strictly greater than". The only exception will be for the building order on partial partitions, where we use $\Subset$ for "less than or equal to" and $\ni$ for "greater than or equal to", without any specific symbol for "strictly less than" and "strictly greater than".

A quasi-order is a reflexive and transitive binary relation. A non-empty intersection of quasi-orders is a quasi-order, and the intersection of an order and a quasi-order is an order.

The set of all partial order relations on a set $P$ is closed under non-void intersection, but it is not a lattice; however, given a fixed partial order $\leq$ on $P$, the set $O(\leq)$ of all partial order relations on $P$ that are included in $\leq$ is a complete lattice, since it is closed under intersection and has a greatest element.

Let $\leq$ be a partial order relation on a set $P$. We call isolated any $x \in P$ that is incomparable to any other element of $P: \forall y \in P$, neither $y<x$ nor $x<y$ holds. For $x, y \in P$ we say that $y$ covers $x$ (or $x$ is covered by $y$ ), and write $x \prec y$ or $y \succ x$, if $x<y$ but there is no $z \in P$ such that $x<z<y$; when we consider the covering relation for distinct orders, we can distinguish them with superscripts like $\stackrel{\times}{\prec}$. Given $x, y \in P$ with $x \leq y$, the length of the interval $[x, y]=\{z \in P \mid x \leq z \leq y\}$ is the supremum of all integers $n$ with $x=z_{0}<\cdots<z_{n}=y$; when this length is finite (for instance when $P$ is finite), it is the greatest such $n$, and the sequence takes the form $x=z_{0} \prec \cdots \prec z_{n}=y$, we call it a covering chain between $x$ and $y$. When $P$ has a least element 0 , the height of $x \in P$ is the length of the interval $[0, x]$. When $P$ has no least element, but for every $x \in P$ there exists a minimal element $m$ such that $m \leq x$, we call the height of $x$ w.r.t. $m$ the length of the interval $[m, x]$.

Given a map $g: P \rightarrow \mathbf{Z}$, we say that $P$ is graded by $g[2,7]$ if for any $x, y \in P$, $x<y \Rightarrow g(x)<g(y)$ and $x \prec y \Rightarrow g(y)=g(x)+1$. Let us now describe the generalization of grading introduced in [16]:

Proposition 1 In a poset $(P, \leq)$, let the covering relation $\prec$ be partitioned into $t$ mutually disjoint non-void relations $\stackrel{1}{\prec}, \ldots, \stackrel{\mathrm{t}}{\prec}$, and consider $t$ maps $g_{1}, \ldots, g_{t}: P \rightarrow \mathbf{Z}$. Suppose that:

1. For all $x, y \in P$ and $i=1, \ldots, t$ we have

$$
x \stackrel{\mathrm{i}}{\prec} y \Longrightarrow\left\{\begin{array}{l}
g_{i}(y)=g_{i}(x)+1 \& \\
g_{j}(y)=g_{j}(x) \text { for } j \neq i .
\end{array}\right.
$$

Then the following two statements are equivalent:

2. Every interval in $P$ has finite length.

3. For all $x, y \in P$,

$$
x<y \Longrightarrow \begin{cases}\forall i \in\{1, \ldots, t\}, & g_{i}(y) \geq g_{i}(x), \& \\ \exists i \in\{1, \ldots, t\}, & g_{i}(y)>g_{i}(x) .\end{cases}
$$

When these conditions are met, we obtain the following:

4. For all $x, y \in P$ and $i=1, \ldots, t$ we have

$$
x \stackrel{\mathrm{i}}{\prec} y \Longleftrightarrow x \leq y \&\left\{\begin{array}{l}
g_{i}(y)=g_{i}(x)+1 \& \\
g_{j}(y)=g_{j}(x) \text { for } j \neq i .
\end{array}\right.
$$


5. In a covering chain $z_{0} \prec \cdots \prec z_{n}$ in $P$, among the $n$ coverings $z_{\ell-1} \prec z_{\ell}(\ell=$ $1, \ldots, n)$, there are $g_{i}\left(z_{n}\right)-g_{i}\left(z_{0}\right)$ occurrences of $z_{\ell-1} \stackrel{\mathrm{i}}{\prec} z_{\ell}$ for $i=1, \ldots, t$.

6. $P$ is graded by $\sum_{i=1}^{t} g_{i}$.

When conditions 1 and 2 (equivalently, 1 and 3) are satisfied, we will say that $P$ is graded by $\left(g_{1}, \ldots, g_{t}\right)$ for $(\stackrel{1}{\prec}, \ldots, \stackrel{\mathrm{t}}{\prec})$. A particular case is when $t=1$, that is, $P$ is graded by $g$ for $\prec$, here conditions 1 and 3 mean that $P$ is graded by $g$; then item 4 gives $x \prec y \Longleftrightarrow[x \leq y \& g(y)=g(x)+1]$, and item 5 means that for $x<y$, all covering chains between $x$ and $y$ have the same length $g(y)-g(x)$ : this is the Jordan-Dedekind chain condition. For $t>1$, item 5 is a stronger version of it.

There is an alternative expression of Proposition 1 in terms of vectors. For $x \in P$, write $G(x)=\left(g_{1}(x) \ldots, g_{t}(x)\right)$; for $i=1, \ldots, t$, let $E_{i} \in \mathbf{Z}^{t}$ be the vector with $i$-th coordinate equal to 1 and all other coordinates equal to 0 . We take the componentwise order on $\mathbf{Z}^{t}$, namely $\left(a_{1}, \ldots, a_{t}\right) \leq\left(b_{1}, \ldots, b_{t}\right)$ iff for $i=1, \ldots, t$ we have $a_{i} \leq b_{i}$, and $\left(a_{1}, \ldots, a_{t}\right)<\left(b_{1}, \ldots, b_{t}\right)$ iff $\left(a_{1}, \ldots, a_{t}\right) \leq\left(b_{1}, \ldots, b_{t}\right)$ and $\left(a_{1}, \ldots, a_{t}\right) \neq\left(b_{1}, \ldots, b_{t}\right)$. Then items 1,3 and 4 can be written as:

1. For all $x, y \in P$ and $i=1, \ldots, t$ we have $x \stackrel{i}{\prec} y \Longrightarrow G(y)=G(x)+E_{i}$.

3. For all $x, y \in P, x<y \Longrightarrow G(x)<G(y)$.

4. For all $x, y \in P$ and $i=1, \ldots, t$ we have $x \stackrel{\text { i }}{\prec} y \Longleftrightarrow\left[x \leq y \& G(y)=G(x)+E_{i}\right]$.

\subsection{Partial partitions, basic and compound orders}

Our general notation follows $[13,14,16]$. A partial partition of $E$ is made of mutually disjoint non-void subsets of $E$ called blocks. We write $\Pi(E)$ for the set of all partitions of $E$, and $\Pi^{*}(E)$ for the set of all partial partitions of $E$; thus $\Pi^{*}(E)=\bigcup_{A \in \mathcal{P}(E)} \Pi(A)$. Write $\emptyset$ for the empty partial partition (with no block); then $\Pi(\emptyset)=\Pi^{*}(\emptyset)=\{\emptyset\}$. Set $\mathbf{1}_{\emptyset}=\mathbf{0}_{\emptyset}=\varnothing$, while for any $A \in \mathcal{P}(E) \backslash\{\emptyset\}$, let $\mathbf{1}_{A}=\{A\}$ (the partition of $A$ into a single block) and $\mathbf{0}_{A}=\{\{p\} \mid p \in A\}$ (the partition of $A$ into its singletons); following [11], we call $\mathbf{0}_{A}$ the identity partition of $A$, and $\mathbf{1}_{A}$ the universal partition of $A$. For $\pi \in \Pi^{*}(E)$, the support of $\pi$, written $\operatorname{supp}(\pi)$, is the union of its blocks: $\operatorname{supp}(\pi)=\bigcup \pi$; the complement of the support is the background of $\pi$, $\operatorname{back}(\pi)=E \backslash \operatorname{supp}(\pi)$. For $\pi \in \Pi^{*}(E)$, a transversal of $\pi$ is a subset of $E$ made by choosing one point in each block of $\pi$, in other words a set $A \subseteq \operatorname{supp}(\pi)$ such that $|A \cap B|=1$ for any $B \in \pi$; a crossing of $\pi$ is a set $A \subseteq \operatorname{supp}(\pi)$ such that $A \cap B \neq \emptyset$ for any $B \in \pi$; in other words, it is a subset of $\operatorname{supp}(\pi)$ containing a transversal of $\pi$.

Let us now recall the order relations on $\Pi^{*}(E)$ considered in [16]. The standard order $\leq$ is given by: $\forall \pi_{1}, \pi_{2} \in \Pi^{*}(E), \pi_{1} \leq \pi_{2} \Longleftrightarrow \forall B \in \pi_{1}, \exists C \in \pi_{2}, B \subseteq C$.

Next, the building order $\Subset[22,23]$ is defined by: $\forall \pi_{1}, \pi_{2} \in \Pi^{*}(E), \pi_{1} \Subset \pi_{2} \Longleftrightarrow$ $\forall C \in \pi_{2}, \exists B \in \pi_{1}, B \subseteq C$.

Note that a block of $\pi_{1}$ is included in at most one block of $\pi_{2}$; on the other hand a block of $\pi_{2}$ can contain several blocks of $\pi_{1}$. We define thus the singularity relation $\Leftarrow[16]$ : for $\pi_{1}, \pi_{2} \in \Pi^{*}(E)$, write $\pi_{1} \Leftarrow \pi_{2}$ (or $\pi_{2} \Rightarrow \pi_{1}$ ) if every block of $\pi_{2}$ contains at most one block of $\pi_{1}$ :

$$
\pi_{1} \Leftarrow \pi_{2} \Longleftrightarrow\left(\forall B, B^{\prime} \in \pi_{1}, \forall C \in \pi_{2},\left[B \subseteq C \& B^{\prime} \subseteq C\right] \Longrightarrow B=B^{\prime}\right) .
$$


In [16] we considered the inclusion order $\subseteq$ and defined the following 4 orders, obtained by intersecting the standard order $\leq$ with the building order $\Subset$, the singularity relation $\Leftarrow$ or the support equality relation:

- The merging order $\stackrel{\mathrm{m}}{\leq}: \forall \pi_{1}, \pi_{2} \in \Pi^{*}(E)$,

$$
\pi_{1} \stackrel{\mathrm{m}}{\leq} \pi_{2} \Longleftrightarrow\left[\pi_{1} \leq \pi_{2} \& \operatorname{supp}\left(\pi_{1}\right)=\operatorname{supp}\left(\pi_{2}\right)\right] .
$$

It is thus the union of the refinement orders on all $\Pi(A), A \in \mathcal{P}(E)$.

- The inflating order $\stackrel{\mathrm{i}}{\leq}: \forall \pi_{1}, \pi_{2} \in \Pi^{*}(E)$,

$$
\pi_{1} \stackrel{i}{\leq} \pi_{2} \Longleftrightarrow\left[\pi_{1} \leq \pi_{2} \& \pi_{1} \Subset \pi_{2} \& \pi_{1} \Leftarrow \pi_{2}\right] .
$$

- The merging-inflating order $\stackrel{\mathrm{mi}}{\leq}: \forall \pi_{1}, \pi_{2} \in \Pi^{*}(E)$,

$$
\pi_{1} \stackrel{\mathrm{mi}}{\leq} \pi_{2} \Longleftrightarrow\left[\pi_{1} \leq \pi_{2} \& \pi_{1} \Subset \pi_{2}\right]
$$

- The inclusion-inflating order $\stackrel{\mathrm{i}}{\subseteq}: \forall \pi_{1}, \pi_{2} \in \Pi^{*}(E)$,

$$
\pi_{1} \stackrel{\mathrm{i}}{\subseteq} \pi_{2} \Longleftrightarrow\left[\pi_{1} \leq \pi_{2} \& \pi_{1} \Leftarrow \pi_{2}\right] .
$$

We next consider the various covering relations on $\Pi^{*}(E)$ associated to the above orders [16]. Given $\pi_{1}, \pi_{2} \in \Pi^{*}(E)$, let us write:

$-\pi_{1} \stackrel{\mathrm{m}}{\prec} \pi_{2}$ if $\pi_{2}$ is obtained by merging two blocks of $\pi_{1}$ :

$$
\pi_{1} \stackrel{\mathrm{m}}{\prec} \pi_{2} \Longleftrightarrow\left[\begin{array}{l}
\left|\pi_{1}\right| \geq 2, \exists C_{1}, C_{2} \in \pi_{1}, C_{1} \neq C_{2}, \\
\pi_{2}=\left(\pi_{1} \backslash\left\{C_{1}, C_{2}\right\}\right) \cup\left\{C_{1} \cup C_{2}\right\}
\end{array}\right] ;
$$

$-\pi_{1} \stackrel{\mathrm{s}}{\prec} \pi_{2}$ if $\pi_{2}$ is obtained by adding a singleton block to $\pi_{1}$ :

$$
\pi_{1} \stackrel{\mathrm{s}}{\prec} \pi_{2} \Longleftrightarrow\left[\operatorname{supp}\left(\pi_{1}\right) \subset E, \exists p \in \operatorname{back}\left(\pi_{1}\right), \pi_{2}=\pi_{1} \cup\{\{p\}\}\right] ;
$$

$-\pi_{1} \stackrel{\mathrm{c}}{\prec} \pi_{2}$ if $\pi_{2}$ is obtained by adding a block to $\pi_{1}$ :

$$
\pi_{1} \stackrel{\mathrm{c}}{\prec} \pi_{2} \Longleftrightarrow\left[\operatorname{supp}\left(\pi_{1}\right) \subset E, \exists B \subseteq \operatorname{back}\left(\pi_{1}\right), B \neq \emptyset, \pi_{2}=\pi_{1} \cup\{B\}\right] ;
$$

$-\pi_{1} \stackrel{i}{\prec} \pi_{2}$ if $\pi_{2}$ is obtained by inflating one block of $\pi_{1}$ by exactly one point:

$$
\pi_{1} \stackrel{\mathrm{i}}{\prec} \pi_{2} \Longleftrightarrow\left[\begin{array}{l}
\operatorname{supp}\left(\pi_{1}\right) \subset E, \pi_{1} \neq \varnothing, \exists p \in \operatorname{back}\left(\pi_{1}\right), \\
\exists B \in \pi_{1}, \pi_{2}=\left(\pi_{1} \backslash\{B\}\right) \cup\{B \cup\{p\}\}
\end{array}\right] .
$$

When $\pi_{1} \stackrel{\times}{\prec} \pi_{2}(x=m, s, c, i)$, we say that $\pi_{2} x$-covers $\pi_{1}$, and we call the relation $\stackrel{\times}{\prec}$ the $x$-covering.

Assume now that $E$ is finite. For any $\pi \in \Pi^{*}(E)$, we define its $m$-height $h_{m}(\pi)$, s-height $h_{s}(\pi)$, and $c$-height $h_{c}(\pi)$, as follows:

$$
h_{c}(\pi)=|\pi|, \quad h_{s}(\pi)=|\operatorname{supp}(\pi)|, \quad h_{m}(\pi)=|\operatorname{supp}(\pi)|-|\pi| .
$$


Table 1 Properties of the 3 basic orders.

\begin{tabular}{l|l|l|l|l|l}
\hline Designation & Maximal & Minimal & Cover & Grading & Height of $\pi$ \\
\hline$\stackrel{\mathrm{m}}{\leq}$ merging & $\mathbf{1}_{A}, A \in \mathcal{P}(E)$ & $\mathbf{0}_{A}, A \in \mathcal{P}(E)$ & $\stackrel{\mathrm{m}}{\prec}$ & $h_{m}$ & w.r.t. $\mathbf{0}_{\operatorname{supp}(\pi)}$ \\
$\subseteq$ inclusion & partitions & $\emptyset$ (least) & $\stackrel{c}{\prec}$ & $h_{c}$ & \\
$\stackrel{\mathrm{i}}{\leq}$ inflating & $\emptyset ;$ partitions & $\mathbf{0}_{A}, A \in \mathcal{P}(E)$ & $\stackrel{\mathrm{i}}{\prec}$ & $h_{m}$ & w.r.t. $\mathbf{0}_{A}, A$ transversal of $\pi$
\end{tabular}

These three heights are non-negative integers. We have

$$
h_{m}(\pi)=h_{s}(\pi)-h_{c}(\pi), \quad h_{s}(\pi)=h_{m}(\pi)+h_{c}(\pi), \quad h_{c}(\pi)=h_{s}(\pi)-h_{m}(\pi) .
$$

Now the height of $\pi$ in the standard order [14] is the sum:

$$
h(\pi)=h_{m}(\pi)+h_{s}(\pi)=2|\operatorname{supp}(\pi)|-|\pi| .
$$

We summarize into Tables 1 and 2 the main properties of the 6 orders studied in [16]. The Maximal and Minimal columns give the maximal and minimal elements; when it is the least or the greatest element, this is indicated by (least) and (greatest) respectively. The 3 compound orders are obtained as a composition of two basic orders, given in the Composition column. The Cover column gives the corresponding covering relation. When $E$ is finite, the Grading column gives the grading function. Basic orders have a simple grading, which gives the height function; however, when there is no least element, we show in the Height of $\pi$ column w.r.t. to which minimal element the height of $\pi$ is given. Compound orders have a double grading, so we indicate also there the function giving the height of $\pi$, it is in fact the sum of the two grading functions.

Table 1 describes the 3 basic orders: merging, inclusion and inflating. Note that for the merging and inflating orders, $\varnothing=\mathbf{1}_{\emptyset}=\mathbf{0}_{\emptyset}$ is isolated. Table 2 (split into two parts) describes the 3 compound orders: merging-inflating, inclusion-inflating and standard. Note that for the merging-inflating order, $\varnothing$ is isolated, and $\mathbf{1}_{E}$ is the greatest element of $\Pi^{*}(E) \backslash\{\varnothing\}$. Also, for the merging-inflating and inclusioninflating orders, the expression as composition of two basic orders commutes.

For the refinement order on $\Pi(E)$, we restrict the merging order to the support $E$, so the greatest and least elements are $\mathbf{1}_{E}$ and $\mathbf{0}_{E}$, the covering relation is $\stackrel{\mathrm{m}}{\prec}$, and when $E$ is finite, $\Pi(E)$ is graded by $h_{m}$, and the latter gives the height function.

\section{Apportioning and derived orders}

We introduce three new orders: the apportioning, apportioning-inflating and extended orders. Since they are based on the building order, we will first briefly study the latter; a more detailed analysis will be given in the Appendix, where we will show that its covering chains have variable length and a strange form, see Figure 15; even by "normalizing" them, their length can still vary, see Figure 16. In Subsection 3.1 we study the first two new orders, both restrict the building order: in the apportioning order, the support remains constant, while in the apportioninginflating order, the support grows. Subsection 3.2 studies the extended order; it 
Table 2 Properties of the 3 compound orders.

\begin{tabular}{|c|c|c|c|}
\hline Designation & Maximal & Minimal & Composition \\
\hline$\leq \operatorname{stan}$ & $\mathbf{1}_{E}$ (greatest) & $\varnothing$ (least) & $\mathrm{m}$ \\
\hline$\stackrel{\text { mi }}{\leq}$ merging-inflating & $\varnothing ; \mathbf{1}_{E}$ & $\mathbf{o}_{A}, A \in \mathcal{P}(E)$ & $\stackrel{m}{\leq} \cdot \stackrel{i}{\leq}$ \\
\hline$\stackrel{i}{\subseteq}$ inclusion-inflating & partitions & $\varnothing$ (least) & $\subseteq$ \\
\hline
\end{tabular}

\begin{tabular}{c|l|c|l}
\hline Order & Cover & Grading & Height of $\pi$ \\
\hline$\leq$ & $\stackrel{\mathrm{m}}{\prec} \cup \stackrel{\mathrm{s}}{\prec}$ & $\left(h_{m}, h_{s}\right)$ for $(\stackrel{\mathrm{m}}{\prec}, \stackrel{\mathrm{s}}{\prec})$ & $h(\pi)$ \\
$\mathrm{mi}$ & $\stackrel{\mathrm{m}}{\prec} \cup \stackrel{\mathrm{i}}{\prec}$ & $\left(-h_{c}, h_{s}\right)$ for $(\stackrel{\mathrm{m}}{\prec}, \stackrel{\mathrm{i}}{\prec})$ & $h_{m}(\pi)$ w.r.t. $\mathbf{0}_{A}, A$ crossing of $\pi$ \\
$\mathrm{i}$ & $\stackrel{\mathrm{s}}{\prec} \cup \stackrel{\mathrm{i}}{\prec}$ & $\left(h_{c}, h_{m}\right)$ for $(\stackrel{\mathrm{s}}{\prec}, \stackrel{\mathrm{i}}{\prec})$ & $h_{s}(\pi)$
\end{tabular}

also involves a growth of the support, but it is not included in the building order. All three orders are graded, with a simple grading for apportioning, and a compound grading for the other two. We will see that they have many similarities with the merging, merging-inflating and standard orders.

Note that the building, apportioning, apportioning-inflating and extended orders do not constitute a lattice on $\Pi^{*}(E)$ : the supremum or infimum of two partial partitions do not necessarily exist. Of all orders considered here and in [16], only the standard order on $\Pi^{*}(E)$ and the refinement order on $\Pi(E)$ are complete lattices.

Recall the definition of the building order $\Subset: \pi_{1} \Subset \pi_{2}$ iff every block of $\pi_{2}$ contains at least one block of $\pi_{1}$. The following properties are known $[22,23,16]$ :

Proposition 2 The building order $\Subset$ is a partial order relation on $\Pi^{*}(E)$. It contains the merging $\stackrel{\mathrm{m}}{\leq}$, inflating $\stackrel{\mathrm{i}}{\leq}$, merging-inflating $\stackrel{\mathrm{mi}}{\leq}$ and inverse inclusion $\supseteq$ orders: for any $\pi_{1}, \pi_{2} \in \Pi^{*}(E)$, each of $\pi_{1} \stackrel{\mathrm{m}}{\leq} \pi_{2}, \pi_{1} \stackrel{\mathrm{i}}{\leq} \pi_{2}, \pi_{1} \stackrel{\mathrm{mi}}{\leq} \pi_{2}$ and $\pi_{1} \supseteq \pi_{2}$ implies $\pi_{1} \Subset \pi_{2}$; we have also

$$
\pi_{1} \leq \pi_{2} \Longrightarrow \pi_{1} \Subset \pi_{2} \backslash \mathcal{P}\left(\operatorname{back}\left(\pi_{1}\right)\right) \Subset \pi_{2} \cap \mathcal{P}\left(\operatorname{supp}\left(\pi_{1}\right)\right) .
$$

Furthermore,

$$
\forall A \in \mathcal{P}(E), \quad \pi_{1} \Subset \pi_{2} \Longrightarrow \pi_{1} \cap \mathcal{P}(A) \Subset \pi_{2} \cap \mathcal{P}(A)
$$

For the analysis of our 3 new orders, we will require the following:

Proposition 3 The building order is generated by inverse inclusion followed by inflating: for any $\pi_{1}, \pi_{2} \in \Pi^{*}(E)$,

$$
\pi_{1} \Subset \pi_{2} \Longleftrightarrow \exists \pi \in \Pi^{*}(E), \pi_{1} \supseteq \pi \stackrel{i}{\leq} \pi_{2}
$$

The least element is $\mathbf{0}_{E}$, the greatest element is $\emptyset$; in $\Pi^{*}(E) \backslash\{\varnothing\}$ (hence in $\Pi(E)$ ), the greatest element is $\mathbf{1}_{E}$. For any $\pi_{0}, \pi_{1}, \pi_{2} \in \Pi^{*}(E)$,

$$
\left[\pi_{0} \Subset \pi_{1} \Subset \pi_{2} \& \pi_{0} \stackrel{i}{\leq} \pi_{2}\right] \Longrightarrow \pi_{0} \stackrel{i}{\leq} \pi_{1} \stackrel{i}{\leq} \pi_{2}
$$




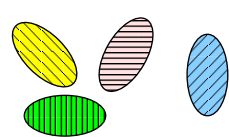

$\pi_{1}$
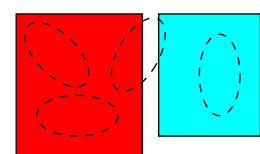

$\pi_{2}$

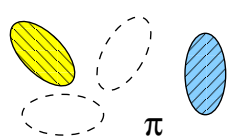

remove
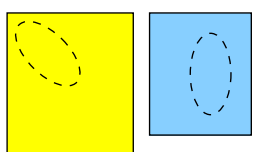

inflate

Fig. 7 Illustration of Proposition 3: from $\pi_{1} \Subset \pi_{2}$ we get $\pi_{1} \supseteq \pi \stackrel{i}{\leq} \pi_{2}$.

Proof If $\pi_{1} \supseteq \pi^{\leq} \leq \pi_{2}$, then by Proposition 2 we get $\pi_{1} \Subset \pi \Subset \pi_{2}$, thus $\pi_{1} \Subset \pi_{2}$. Conversely, let $\pi_{1} \Subset \pi_{2}$. For each $C \in \pi_{2}$, choose one $B \in \pi_{1}$ such that $B \subseteq C$, and set $f(C)=B$. Let $\pi=\left\{f(C) \mid C \in \pi_{2}\right\}$; then $\pi_{1} \supseteq \pi$; as $f(C) \subseteq C$ for all $C \in \pi_{2}$, $\pi \leq \pi_{2}$ and $\pi \Subset \pi_{2}$ by construction; now for any other $D \in \pi_{2}$, as $f(D) \subseteq D$ and $D \cap C=\emptyset$, we have $f(D) \nsubseteq C$, hence $\pi \Leftarrow \pi_{2}$. Thus $\pi \stackrel{i}{\leq} \pi_{2}$ by (2). This construction $\pi_{1} \supseteq \pi \stackrel{i}{\leq} \pi_{2}$ is illustrated in Figure 7 .

In a partial partition $\pi$, every block of $\pi$ contains at least one singleton $\{p\}$; hence $\mathbf{0}_{E} \Subset \pi$. As $\varnothing$ has no block, $\pi \Subset \varnothing$. When $\pi \in \Pi^{*}(E) \backslash\{\varnothing\}, \pi$ has at least one block $B$, included in $E$, so $\pi \Subset \mathbf{1}_{E}$, with $\mathbf{1}_{E} \in \Pi(E)$.

Let $\pi_{0}, \pi_{1}, \pi_{2} \in \Pi^{*}(E)$ such that $\pi_{0} \Subset \pi_{1} \Subset \pi_{2}$ and $\pi_{0} \stackrel{i}{\leq} \pi_{2}$; thus $\pi_{0} \leq \pi_{2}$ and $\pi_{0} \Leftarrow \pi_{2}$. We first show that for any $A \in \pi_{0}$, there exist $B_{A} \in \pi_{1}$ and $C_{A} \in \pi_{2}$ such that $A \subseteq B_{A} \subseteq C_{A}$. Indeed, as $\pi_{0} \leq \pi_{2}$, there is $C_{A} \in \pi_{2}$ such that $A \subseteq C_{A}$; as $\pi_{1} \Subset \pi_{2}$, there is $B_{A} \in \pi_{1}$ such that $B_{A} \subseteq C_{A}$; as $\pi_{0} \Subset \pi_{1}$, there is $A^{\prime} \in \pi_{0}$ such that $A^{\prime} \subseteq B_{A}$; now $A, A^{\prime} \in \pi_{0}$ and $A, A^{\prime} \subseteq C_{A}$; as $\pi_{0} \Leftarrow \pi_{2}$, we get $A=A^{\prime}$, hence $A \subseteq B_{A} \subseteq C_{A}$. It follows that $\pi_{0} \leq \pi_{1}$. We next show that for any $B \in \pi_{1}$ there exists $C \in \pi_{2}$ such that $B \subseteq C$. Indeed, as $\pi_{0} \Subset \pi_{1}$, there is $A \in \pi_{0}$ such that $A \subseteq B$; by the above, there are $B_{A} \in \pi_{1}$ and $C_{A} \in \pi_{2}$ such that $A \subseteq B_{A} \subseteq C_{A}$; but $B, B_{A} \in \pi_{1}$ with $\emptyset \neq A \subseteq B, B_{A}$, so $B_{A}=B$; hence $B \subseteq C_{A}$. It follows that $\pi_{1} \leq \pi_{2}$. Since $\pi_{0} \leq \pi_{1} \leq \pi_{2}$ and $\pi_{0} \Subset \pi_{1} \Subset \pi_{2}$, by (3) we have $\pi_{0} \stackrel{\text { mi }}{\leq} \pi_{1} \stackrel{\text { mi }}{\leq} \pi_{2}$; now $\pi_{0} \stackrel{i}{\leq} \pi_{2}$, so Equation (26) in Theorem 12 of [16] gives $\pi_{0} \stackrel{i}{\leq} \pi_{1} \stackrel{i}{\leq} \pi_{2}$.

We see that for the building order, the growth of a partial partition is achieved by first removing some blocks, then inflating some of the remaining blocks. This is exactly what is done in Serra's method $[22,23]$ for eliminating "small parasitic" segmentation classes, which we described in the Introduction. Since the merging order is included in the building order, a particular case is that merging blocks can be achieved by removing some blocks then inflating the remaining blocks, an elementary fact proven in item 2 of Proposition 10 of [16]. However, when $\pi_{1} \supseteq \pi \stackrel{\mathrm{i}}{\leq} \pi_{2}$, we have indeed $\pi_{1} \Subset \pi \Subset \pi_{2}$, but also $\pi_{1} \geq \pi \leq \pi_{2}$; it is thus somewhat counterintuitive to have two successive growths of a partial partition where the support first decreases from $\pi_{1}$ to $\pi$, next increases from $\pi$ to $\pi_{2}$. In the Appendix we will exhibit some bizarre properties of the building order, in particular its strange covering chains, see Figure 15. In our opinion, this is probably related to the fact that the building order involves no constraint on the support of partial partitions. This will not happen with our three new orders: the apportioning order requires support equality $\operatorname{supp}\left(\pi_{1}\right)=\operatorname{supp}\left(\pi_{2}\right)$, while the merging-apportioning and extended orders require support inclusion $\operatorname{supp}\left(\pi_{1}\right) \subseteq \operatorname{supp}\left(\pi_{2}\right)$. 


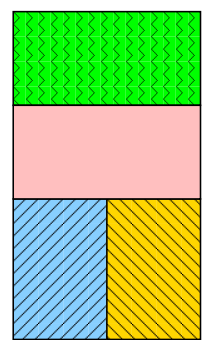

$\pi_{1}$

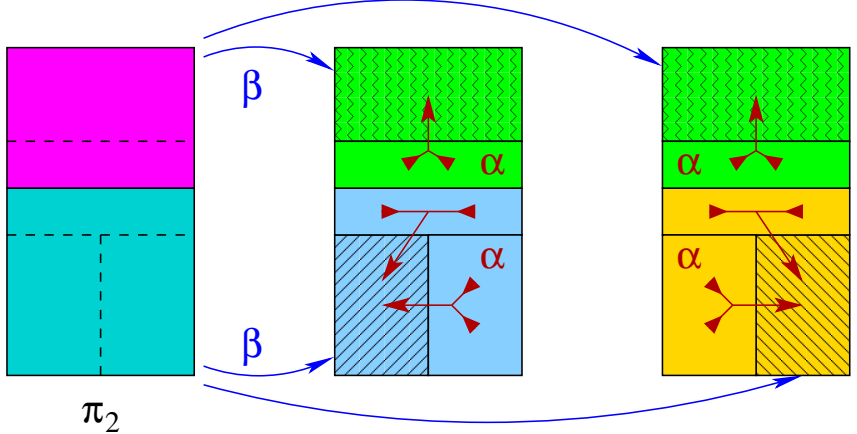

Fig. 8 From left to right: first $\pi_{1}$, second $\pi_{2}$; here $\pi_{1} \stackrel{\text { a }}{\leq} \pi_{2}$, the blocks of $\pi_{1}$ included in a block of $\pi_{2}$ are hatched. Third and fourth, two possible choices for $\beta$, and the resulting $\alpha$, in order to apportion $\pi_{1}$ into $\pi_{2}$.

\subsection{Apportioning as a generalization of block merging}

We will study apportioning from two points of view: first as an operation that generalizes block merging, as it splits some blocks and merges their parts with other blocks; next as an order relation on $\Pi^{*}(E)$ built from existing orders and quasi-orders. This double point of view, in terms of operations on blocks and of order relations, will also apply to the apportioning-inflating order.

Let $\pi_{1}$ and $\pi_{2}$ be two non-empty partial partitions having the same support, and let $\pi_{2}$ be obtained from $\pi_{1}$ by apportioning some of its blocks. We will decompose $\pi_{1}$ into a set $\pi_{a}$ of blocks to be apportioned, and the non-empty set $\pi_{r}=\pi_{1} \backslash \pi_{a}$ of remaining blocks. A block of $\pi_{1}$ that is split into parts will belong to $\pi_{a}$, and each part will be merged with a block of $\pi_{r}$; when several blocks of $\pi_{1}$ are merged, one chooses one of them to be in $\pi_{r}$, and the others will be in $\pi_{a}$. Thus each block of $\pi_{2}$ will be made of one block of $\pi_{r}$, to which we add entire blocks, or parts of blocks, of $\pi_{a}$. Hence a block of $\pi_{2}$ contains at least one block of $\pi_{1}$, but exactly one of them belongs to $\pi_{r}$; when this block of $\pi_{2}$ contains several blocks $B_{1}, \ldots, B_{m}$ of $\pi_{1}$, we must choose one $B_{i}$ to be remaining, $B_{i} \in \pi_{r}$, and the other ones will be apportioned, $B_{j} \in \pi_{a}$ for $j \neq i$. Thus the decomposition of $\pi_{1}$ into $\pi_{a}$ and $\pi_{r}$ is not necessarily unique. So we postulate a map $\beta: \pi_{2} \rightarrow \pi_{1}$ that chooses for each block $C \in \pi_{2}$, among all blocks $B \in \pi_{1}$ such that $B \subseteq C$, the one that will belong to $\pi_{r}$; thus $\beta(C) \subseteq C$. Since a block of $\pi_{1}$ cannot belong to several blocks of $\pi_{2}, \beta$ will be injective. Then $\pi_{r}=\left\{\beta(C) \mid C \in \pi_{2}\right\}=\beta\left(\pi_{2}\right)$ and $\pi_{a}=\pi_{1} \backslash \beta\left(\pi_{2}\right)$.

In order to describe the apportioning, we must indicate for every $p \in \operatorname{supp}\left(\pi_{a}\right)$ to which block of $\pi_{r}$ it will be added; this block will be written $\alpha(p)$. We define thus a map $\alpha: \operatorname{supp}\left(\pi_{a}\right) \rightarrow \pi_{r}$ implementing this apportioning, namely each block $B \in \pi_{r}$ will be enlarged by adding to it $\alpha^{-1}(B)=\left\{p \in \operatorname{supp}\left(\pi_{a}\right) \mid \alpha(p)=B\right\}$. As $\pi_{r}$ and $\pi_{a}$ are given in terms of the map $\beta$, we have $\alpha: \operatorname{supp}\left(\pi_{1} \backslash \beta\left(\pi_{2}\right)\right) \rightarrow \beta\left(\pi_{2}\right)$, and each block $B \in \beta\left(\pi_{2}\right)$ gives rise to a block $B \cup \alpha^{-1}(B) \in \pi_{2}$. Conversely, for every $C \in \pi_{2}, C=\beta(C) \cup \alpha^{-1}(\beta(C))$, where $\beta(C)$ and $\alpha^{-1}(\beta(C))$ are disjoint. See Figure 8. Thus $\pi_{2}=\left\{B \cup \alpha^{-1}(B) \mid B \in \beta\left(\pi_{2}\right)\right\}=\left\{\beta(C) \cup \alpha^{-1}(\beta(C)) \mid C \in \pi_{2}\right\}$. Note that for $C \in \pi_{2}$ and $B \in \pi_{1} \backslash \beta\left(\pi_{2}\right)$, the portion of $B$ apportioned to $\beta(C)$ is $B \cap C=B \cap \alpha^{-1}(\beta(C))$. 
In fact, only the injection $\beta: \pi_{2} \rightarrow \pi_{1}$ really matters, since $\alpha$ can be uniquely determined from $\pi_{1}, \pi_{2}$ and $\beta$ :

Lemma 4 Let $\pi_{1}, \pi_{2} \in \Pi^{*}(E) \backslash\{\varnothing\}$ and $\beta: \pi_{2} \rightarrow \pi_{1}$. Then the following two conditions are equivalent:

1. $\operatorname{supp}\left(\pi_{1}\right)=\operatorname{supp}\left(\pi_{2}\right)$ and for every $C \in \pi_{2}, \beta(C) \subseteq C$.

2. There is a map $\alpha: \operatorname{supp}\left(\pi_{1} \backslash \beta\left(\pi_{2}\right)\right) \rightarrow \beta\left(\pi_{2}\right)$ such that for every $C \in \pi_{2}, C=$ $\beta(C) \cup \alpha^{-1}(\beta(C))$.

Furthermore, $\beta$ is an injection $\pi_{2} \rightarrow \pi_{1}$, and for every $C \in \pi_{2}$ we have:

$$
\begin{aligned}
C \cap \operatorname{supp}\left(\beta\left(\pi_{2}\right)\right) & =\beta(C), \\
C \cap \operatorname{supp}\left(\pi_{1} \backslash \beta\left(\pi_{2}\right)\right)=C \backslash \operatorname{supp}\left(\beta\left(\pi_{2}\right)\right) & =C \backslash \beta(C) .
\end{aligned}
$$

Finally, the map $\alpha$ of item 2 is unique, it is given by:

$$
\forall p \in \operatorname{supp}\left(\pi_{1} \backslash \beta\left(\pi_{2}\right)\right), \forall C \in \pi_{2}, \quad[\alpha(p)=\beta(C) \Longleftrightarrow p \in C] .
$$

Proof First, suppose condition 1: $\operatorname{supp}\left(\pi_{1}\right)=\operatorname{supp}\left(\pi_{2}\right)$ and $\beta(C) \subseteq C$ for all $C \in \pi_{2}$. For two distinct $C, D \in \pi_{2}$ we have $\beta(C) \subseteq C, \beta(D) \subseteq D$ and $C \cap D=\emptyset$, so $\beta(C) \neq \beta(D)$; hence $\beta$ is injective. Fix $C \in \pi_{2}$; for any block $D \in \pi_{2}$ distinct from $C, \beta(D) \cap C \subseteq D \cap C=\emptyset$; thus

$$
C \cap \operatorname{supp}\left(\beta\left(\pi_{2}\right)\right)=C \cap\left(\bigcup_{D \in \pi_{2}} \beta(D)\right)=\bigcup_{D \in \pi_{2}}(C \cap \beta(D))=C \cap \beta(C)=\beta(C) .
$$

Then $C \backslash \operatorname{supp}\left(\beta\left(\pi_{2}\right)\right)=C \backslash\left(C \cap \operatorname{supp}\left(\beta\left(\pi_{2}\right)\right)\right)=C \backslash \beta(C)$. Now

$$
\operatorname{supp}\left(\pi_{1} \backslash \beta\left(\pi_{2}\right)\right)=\operatorname{supp}\left(\pi_{1}\right) \backslash \operatorname{supp}\left(\beta\left(\pi_{2}\right)\right)=\operatorname{supp}\left(\pi_{2}\right) \backslash \operatorname{supp}\left(\beta\left(\pi_{2}\right)\right),
$$

with $C \subseteq \operatorname{supp}\left(\pi_{2}\right)$. Hence (13) holds.

For $p \in \operatorname{supp}\left(\pi_{1} \backslash \beta\left(\pi_{2}\right)\right), p \in \operatorname{supp}\left(\pi_{1}\right)=\operatorname{supp}\left(\pi_{2}\right)$, so $p$ belongs to a unique block of $\pi_{2}$, and we can define $\alpha: \operatorname{supp}\left(\pi_{1} \backslash \beta\left(\pi_{2}\right)\right) \rightarrow \beta\left(\pi_{2}\right)$ by (14). Take $C \in \pi_{2}$. For $p \in \alpha^{-1}(\beta(C))$, we have $\alpha(p)=\beta(C)$, so $p \in C$ by $(14)$; thus $\alpha^{-1}(\beta(C)) \subseteq C$. For $p \in C \backslash \beta(C), p \in \operatorname{supp}\left(\pi_{1} \backslash \beta\left(\pi_{2}\right)\right)$ by $(13)$, so $\alpha(p)$ is defined; as $p \in C$, (14) gives $\alpha(p)=\beta(C)$, so $p \in \alpha^{-1}(\beta(C))$; thus $C \backslash \beta(C) \subseteq \alpha^{-1}(\beta(C))$. From the inequalities $\beta(C) \subseteq C$ and $C \backslash \beta(C) \subseteq \alpha^{-1}(\beta(C)) \subseteq C$, we deduce that $C=\beta(C) \cup \alpha^{-1}(\beta(C))$. Therefore condition 2 holds.

Next, suppose condition 2: we have $\alpha: \operatorname{supp}\left(\pi_{1} \backslash \beta\left(\pi_{2}\right)\right) \rightarrow \beta\left(\pi_{2}\right)$ such that for all $C \in \pi_{2}, C=\beta(C) \cup \alpha^{-1}(\beta(C))$; then $\beta(C) \subseteq \beta(C) \cup \alpha^{-1}(\beta(C))=C$. Since $\alpha$ is $\operatorname{supp}\left(\pi_{1} \backslash \beta\left(\pi_{2}\right)\right) \rightarrow \beta\left(\pi_{2}\right)$, we have

$$
\bigcup_{C \in \pi_{2}} \alpha^{-1}(\beta(C))=\alpha^{-1}\left(\beta\left(\pi_{2}\right)\right)=\operatorname{supp}\left(\pi_{1} \backslash \beta\left(\pi_{2}\right)\right)=\operatorname{supp}\left(\pi_{1}\right) \backslash \operatorname{supp}\left(\beta\left(\pi_{2}\right)\right) ;
$$

thus

$$
\begin{aligned}
\operatorname{supp}\left(\pi_{2}\right) & =\bigcup_{C \in \pi_{2}}\left[\beta(C) \cup \alpha^{-1}(\beta(C))\right]=\left[\bigcup_{C \in \pi_{2}} \beta(C)\right] \cup\left[\bigcup_{C \in \pi_{2}} \alpha^{-1}(\beta(C))\right] \\
& =\operatorname{supp}\left(\beta\left(\pi_{2}\right)\right) \cup\left(\operatorname{supp}\left(\pi_{1}\right) \backslash \operatorname{supp}\left(\beta\left(\pi_{2}\right)\right)\right)=\operatorname{supp}\left(\pi_{1}\right) .
\end{aligned}
$$

Therefore condition 1 holds. 
Let us now show that $\alpha$ satisfies (14). Let $C \in \pi_{2}$ and $p \in \operatorname{supp}\left(\pi_{1} \backslash \beta\left(\pi_{2}\right)\right)$. If $\alpha(p)=\beta(C)$, then $p \in \alpha^{-1}(\beta(C))$; but $C=\beta(C) \cup \alpha^{-1}(\beta(C))$, thus $p \in C$. Now if $\alpha(p) \neq \beta(C)$, then there is some $D \in \pi_{2}$ such that $\alpha(p)=\beta(D)$, and we have $D \neq C$; by the argument in the previous sentence, $p \in D$, and as $C \cap D=\emptyset$, we get $p \notin C$. Therefore $\alpha(p)=\beta(C) \Longleftrightarrow p \in C$ and (14) holds.

A direct consequence of (13) is that $\operatorname{supp}\left(\pi_{1} \backslash \beta\left(\pi_{2}\right)\right)=\bigcup_{C \in \pi_{2}}(C \backslash \beta(C))$. When the conditions of Lemma 4 are satisfied, we will say that $\beta$ apportions $\pi_{1}$ into $\pi_{2}$. We now define the apportioning order $\stackrel{\text { a }}{\leq}$ mathematically as the intersection of the building order and the support equality relation:

$$
\forall \pi_{1}, \pi_{2} \in \Pi^{*}(E), \quad \pi_{1} \stackrel{\mathrm{a}}{\leq} \pi_{2} \Longleftrightarrow\left[\pi_{1} \Subset \pi_{2} \& \operatorname{supp}\left(\pi_{1}\right)=\operatorname{supp}\left(\pi_{2}\right)\right] .
$$

Note that here we do not require $\pi_{1}$ and $\pi_{2}$ to be non-empty; in fact when $\pi_{1} \stackrel{\text { a }}{\leq} \pi_{2}$, $\pi_{1}=\varnothing \Leftrightarrow \pi_{2}=\varnothing$. The two versions of apportioning are equivalent:

Proposition 5 Given $\pi_{1}, \pi_{2} \in \Pi^{*}(E) \backslash\{\varnothing\}$, we have $\pi_{1} \stackrel{\text { a }}{\leq} \pi_{2}$ if and only if there exists $\beta: \pi_{2} \rightarrow \pi_{1}$ such that $\beta$ apportions $\pi_{1}$ into $\pi_{2}$.

Proof If $\beta: \pi_{2} \rightarrow \pi_{1}$ apportions $\pi_{1}$ into $\pi_{2}$, then $\operatorname{supp}\left(\pi_{1}\right)=\operatorname{supp}\left(\pi_{2}\right)$ and for every $C \in \pi_{2}, \beta(C) \subseteq C$; as $\beta(C) \in \pi_{1}$, this means that $\pi_{1} \Subset \pi_{2}$. Hence $\pi_{1} \stackrel{\text { a }}{\leq} \pi_{2}$.

Conversely, if $\pi_{1} \stackrel{\text { a }}{\leq} \pi_{2}$, then $\operatorname{supp}\left(\pi_{1}\right)=\operatorname{supp}\left(\pi_{2}\right)$ and $\pi_{1} \Subset \pi_{2}$. Thus for every $C \in \pi_{2}$ there exists at least one $B \in \pi_{1}$ such that $B \subseteq C$; we choose one such $B$ and set $\beta(C)=B$. Then $\beta$ is a map $\pi_{2} \rightarrow \pi_{1}$ and $\beta(C) \subseteq C$ for all $C \in \pi_{2}$. Hence $\beta$ apportions $\pi_{1}$ into $\pi_{2}$.

We have $\pi_{1} \supseteq \beta\left(\pi_{2}\right) \stackrel{i}{\leq} \pi_{2}$ (cf. Proposition 3 ). As explained above, the map $\beta$ : $\pi_{2} \rightarrow \pi_{1}$ is not necessarily unique, see Figure 8 . However the number of apportioned blocks, that is, the size of $\pi_{1} \backslash \beta\left(\pi_{2}\right)$, remains constant:

Lemma 6 Let $\pi_{1}, \pi_{2} \in \Pi^{*}(E) \backslash\{\varnothing\}$, and $\beta: \pi_{2} \rightarrow \pi_{1}$ such that $\beta$ apportions $\pi_{1}$ into $\pi_{2}$. Then:

1. $\pi_{1}=\pi_{2} \Longleftrightarrow \beta\left(\pi_{2}\right)=\pi_{1} \Longleftrightarrow \beta\left(\pi_{2}\right)=\pi_{2}$.

2. For any other $\beta^{\prime}: \pi_{2} \rightarrow \pi_{1}$ such that $\beta^{\prime}$ apportions $\pi_{1}$ into $\pi_{2}$, there is a bijection between $\pi_{1} \backslash \beta^{\prime}\left(\pi_{2}\right)$ and $\pi_{1} \backslash \beta\left(\pi_{2}\right)$.

Proof 1. We have

$$
\beta\left(\pi_{2}\right)=\pi_{1} \Longleftrightarrow \pi_{1} \backslash \beta\left(\pi_{2}\right)=\varnothing \Longleftrightarrow \operatorname{supp}\left(\pi_{1} \backslash \beta\left(\pi_{2}\right)\right)=\emptyset .
$$

Let $\alpha: \operatorname{supp}\left(\pi_{1} \backslash \beta\left(\pi_{2}\right)\right) \rightarrow \beta\left(\pi_{2}\right)$ be the map associated to $\beta$ in Lemma 4. If $\beta\left(\pi_{2}\right)=\pi_{1}$, then for any $C \in \pi_{2}$ we have $\alpha^{-1}(\beta(C)) \subseteq \operatorname{supp}\left(\pi_{1} \backslash \beta\left(\pi_{2}\right)\right)=\emptyset$, so $C=\beta(C) \cup \alpha^{-1}(\beta(C))=\beta(C)$, and we get $\beta\left(\pi_{2}\right)=\pi_{2}$. Hence $\pi_{1}=\beta\left(\pi_{2}\right)=\pi_{2}$.

If $\beta\left(\pi_{2}\right) \neq \pi_{1}$, then $\operatorname{supp}\left(\pi_{1} \backslash \beta\left(\pi_{2}\right)\right) \neq \emptyset$, so there exists $p \in \operatorname{supp}\left(\pi_{1} \backslash \beta\left(\pi_{2}\right)\right)$, and we get $\alpha(p)=\beta(C)$ for some $C \in \pi_{2}$; thus $\alpha^{-1}(\beta(C)) \neq \emptyset$, so $\beta(C) \subset \beta(C) \cup$ $\alpha^{-1}(\beta(C))=C$; this means that $\beta\left(\pi_{2}\right) \neq \pi_{2}$, and as $\beta(C) \in \pi_{1}, \pi_{1} \neq \pi_{2}$.

2. For any $C \in \pi_{2}$, if $\beta(C)=\beta^{\prime}(C)$, then $\beta(C)=\beta^{\prime}(C) \in \beta\left(\pi_{2}\right) \cap \beta^{\prime}\left(\pi_{2}\right)$. Conversely, if $B \in \beta\left(\pi_{2}\right) \cap \beta^{\prime}\left(\pi_{2}\right)$, then there are $C, D \in \pi_{2}$ such that $B=\beta(C)=$ 
$\beta^{\prime}(D)$; but $\beta(C) \subseteq C$ and $\beta^{\prime}(D) \subseteq D$, so $B \subseteq C \cap D$, hence $C=D$ and $B=\beta(C)=$ $\beta^{\prime}(C)$. Thus for $C \in \pi_{2}$, if $\beta(C) \neq \beta^{\prime}(C)$, then $\beta(C), \beta^{\prime}(C) \notin \beta\left(\pi_{2}\right) \cap \beta^{\prime}\left(\pi_{2}\right)$, hence $\beta(C) \in \beta\left(\pi_{2}\right) \backslash \beta^{\prime}\left(\pi_{2}\right)$ and $\beta^{\prime}(C) \in \beta^{\prime}\left(\pi_{2}\right) \backslash \beta\left(\pi_{2}\right)$. Therefore we have a bijection between $\beta\left(\pi_{2}\right) \backslash \beta^{\prime}\left(\pi_{2}\right)$ and $\beta^{\prime}\left(\pi_{2}\right) \backslash \beta\left(\pi_{2}\right)$, associating $\beta(C)$ to $\beta^{\prime}(C)$ for all $C \in \pi_{2}$ such that $\beta(C) \neq \beta^{\prime}(C)$. Combining it with the identity on $\pi_{1} \backslash\left(\beta\left(\pi_{2}\right) \cup \beta^{\prime}\left(\pi_{2}\right)\right)$, we get a bijection between

$$
\pi_{1} \backslash \beta^{\prime}\left(\pi_{2}\right)=\left(\beta\left(\pi_{2}\right) \backslash \beta^{\prime}\left(\pi_{2}\right)\right) \cup\left[\pi_{1} \backslash\left(\beta\left(\pi_{2}\right) \cup \beta^{\prime}\left(\pi_{2}\right)\right)\right]
$$

and

$$
\pi_{1} \backslash \beta\left(\pi_{2}\right)=\left(\beta^{\prime}\left(\pi_{2}\right) \backslash \beta\left(\pi_{2}\right)\right) \cup\left[\pi_{1} \backslash\left(\beta\left(\pi_{2}\right) \cup \beta^{\prime}\left(\pi_{2}\right)\right)\right] .
$$

Let $\pi_{1}, \pi_{2} \in \Pi^{*}(E) \backslash\{\varnothing\}$. By item 2 , when $\pi_{1} \stackrel{\text { a }}{\leq} \pi_{2}$, for any $\beta: \pi_{2} \rightarrow \pi_{1}$ that apportions $\pi_{1}$ into $\pi_{2}$, the cardinal $\left|\pi_{1} \backslash \beta\left(\pi_{2}\right)\right|$, which gives the number of blocks that are apportioned to other blocks, will remain constant. We call that constant cardinal the apportioning index of $\pi_{1}$ into $\pi_{2}$, and write it $I\left(\pi_{1}, \pi_{2}\right)$. We also trivially define $I(\varnothing, \varnothing)=0$.

By item 1, if $\pi_{1}=\pi_{2}$ we have $\pi_{1}=\beta\left(\pi_{2}\right)=\pi_{2}$, while if $\pi_{1} \stackrel{\text { a }}{<} \pi_{2}$ we have $\pi_{1} \supset \beta\left(\pi_{2}\right) \stackrel{i}{<} \pi_{2}$. Thus

$$
\pi_{1} \stackrel{\mathrm{a}}{<} \pi_{2} \Longleftrightarrow\left[\operatorname{supp}\left(\pi_{1}\right)=\operatorname{supp}\left(\pi_{2}\right) \& \exists \pi \in \Pi^{*}(E), \pi_{1} \supset \pi \stackrel{\mathrm{i}}{<} \pi_{2}\right] .
$$

Also $I\left(\pi_{1}, \pi_{2}\right)=0 \Longleftrightarrow \pi_{1}=\pi_{2}$ (this remains true for $\pi_{1}=\pi_{2}=\varnothing$.)

Now in a composition of apportionings, the apportioning indexes add up:

Proposition 7 Let $\pi_{0}, \pi_{1}, \pi_{2} \in \Pi^{*}(E) \backslash\{\varnothing\}$. Suppose that $\beta_{1}$ apportions $\pi_{0}$ into $\pi_{1}$ and $\beta_{2}$ apportions $\pi_{1}$ into $\pi_{2}$. Then $\beta_{1} \beta_{2}$ apportions $\pi_{0}$ into $\pi_{2}$, and $I\left(\pi_{0}, \pi_{2}\right)=$ $I\left(\pi_{0}, \pi_{1}\right)+I\left(\pi_{1}, \pi_{2}\right)$.

Let $\alpha_{1}: \operatorname{supp}\left(\pi_{0} \backslash \beta_{1}\left(\pi_{1}\right)\right) \rightarrow \beta\left(\pi_{2}\right), \alpha_{2}: \operatorname{supp}\left(\pi_{1} \backslash \beta_{2}\left(\pi_{2}\right)\right) \rightarrow \beta\left(\pi_{2}\right)$ and $\alpha_{12}:$ $\operatorname{supp}\left(\pi_{0} \backslash \beta_{1} \beta_{2}\left(\pi_{2}\right)\right) \rightarrow \beta_{1} \beta_{2}\left(\pi_{2}\right)$ be the maps associated to $\beta_{1}, \beta_{2}$ and $\beta_{1} \beta_{2}$ respectively, according to (14). The domain of definition of $\alpha_{12}$ is the union of those of $\alpha_{1}$ and $\alpha_{2}$ :

$$
\operatorname{supp}\left(\pi_{0} \backslash \beta_{1} \beta_{2}\left(\pi_{2}\right)\right)=\operatorname{supp}\left(\pi_{0} \backslash \beta_{1}\left(\pi_{1}\right)\right) \cup \operatorname{supp}\left(\pi_{1} \backslash \beta_{2}\left(\pi_{2}\right)\right) .
$$

For $p \in \operatorname{supp}\left(\pi_{0} \backslash \beta_{1} \beta_{2}\left(\pi_{2}\right)\right), \alpha_{12}(p)$ depends on the last apportioning to which the $p$ is subjected (see Figure 9):

1. If $p$ belongs to a block of $\pi_{0}$ that is apportioned and to a block of $\pi_{1}$ that is enlarged, i.e., if $p \in \operatorname{supp}\left(\pi_{0} \backslash \beta_{1}\left(\pi_{1}\right)\right)$ and $p \notin \operatorname{supp}\left(\pi_{1} \backslash \beta_{2}\left(\pi_{2}\right)\right)$, then $\alpha_{12}(p)=\alpha_{1}(p)$.

2. If $p$ belongs to a block of $\pi_{1}$ that is apportioned, i.e., if $p \in \operatorname{supp}\left(\pi_{1} \backslash \beta_{2}\left(\pi_{2}\right)\right)$, then $\alpha_{12}(p)=\beta_{1} \alpha_{2}(p)$.

Proof Clearly $\beta_{1} \beta_{2}$ is an injection $\pi_{2} \rightarrow \pi_{0}$ and for every $C \in \pi_{2}, \beta_{1} \beta_{2}(C) \subseteq$ $\beta_{2}(C) \subseteq C$. Also $\operatorname{supp}\left(\pi_{0}\right)=\operatorname{supp}\left(\pi_{1}\right)=\operatorname{supp}\left(\pi_{2}\right)$. Thus $\beta_{1} \beta_{2}$ apportions $\pi_{0}$ into $\pi_{2}$.

Since $\beta_{1}$ is injective, its restriction to $\pi_{1} \backslash \beta_{2}\left(\pi_{2}\right)$ is a bijection $\pi_{1} \backslash \beta_{2}\left(\pi_{2}\right) \rightarrow$ $\beta_{1}\left(\pi_{1}\right) \backslash \beta_{1} \beta_{2}\left(\pi_{2}\right)$, so $\left|\pi_{1} \backslash \beta_{2}\left(\pi_{2}\right)\right|=\left|\beta_{1}\left(\pi_{1}\right) \backslash \beta_{1} \beta_{2}\left(\pi_{2}\right)\right|$. Now $\pi_{0} \backslash \beta_{1} \beta_{2}\left(\pi_{2}\right)$ is the disjoint union of $\pi_{0} \backslash \beta_{1}\left(\pi_{1}\right)$ and $\beta_{1}\left(\pi_{1}\right) \backslash \beta_{1} \beta_{2}\left(\pi_{2}\right)$. Thus

$$
\begin{gathered}
I\left(\pi_{0}, \pi_{2}\right)=\left|\pi_{0} \backslash \beta_{1} \beta_{2}\left(\pi_{2}\right)\right|=\left|\pi_{0} \backslash \beta_{1}\left(\pi_{1}\right)\right|+\left|\beta_{1}\left(\pi_{1}\right) \backslash \beta_{1} \beta_{2}\left(\pi_{2}\right)\right| \\
=\left|\pi_{0} \backslash \beta_{1}\left(\pi_{1}\right)\right|+\left|\pi_{1} \backslash \beta_{2}\left(\pi_{2}\right)\right|=I\left(\pi_{0}, \pi_{1}\right)+I\left(\pi_{1}, \pi_{2}\right) .
\end{gathered}
$$




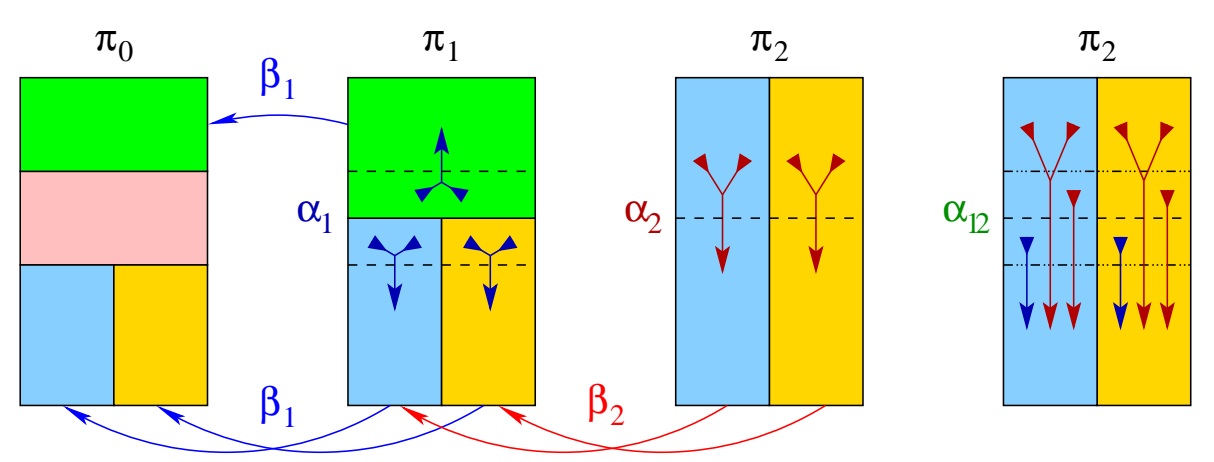

Fig. 9 From left to right: $\pi_{0}$, then $\pi_{1}$, with $\beta_{1}$ apportioning $\pi_{0}$ into $\pi_{1}$, next $\pi_{2}$, with $\beta_{2}$ apportioning $\pi_{1}$ into $\pi_{2}$. Finally we show the apportioning of $\pi_{0}$ into $\pi_{2}$, with the map $\alpha_{12}$ associated to $\beta_{1} \beta_{2}$, given by $\alpha_{12}(p)=\alpha_{1}(p)$ for $p \in \operatorname{supp}\left(\beta_{2}\left(\pi_{2}\right)\right) \backslash \operatorname{supp}\left(\beta_{1}\left(\pi_{1}\right)\right)$, and $\alpha_{12}(p)=$ $\beta_{1} \alpha_{2}(p)$ for $p \in \operatorname{supp}\left(\pi_{0}\right) \backslash \operatorname{supp}\left(\beta_{2}\left(\pi_{2}\right)\right)$.

Let $p \in \operatorname{supp}\left(\pi_{0}\right)$. If $p \in \operatorname{supp}\left(\pi_{0} \backslash \beta_{1}\left(\pi_{1}\right)\right)$, then we have $p \notin \beta_{1}(B)$ for all $B \in \pi_{1}$, in particular for any $C \in \pi_{2}, \beta_{2}(C) \in \pi_{1}$, so $p \notin \beta_{1} \beta_{2}(C)$, hence $p \in \operatorname{supp}\left(\pi_{0} \backslash\right.$ $\left.\beta_{1} \beta_{2}\left(\pi_{2}\right)\right)$; thus (a) $\operatorname{supp}\left(\pi_{0} \backslash \beta_{1}\left(\pi_{1}\right)\right) \subseteq \operatorname{supp}\left(\pi_{0} \backslash \beta_{1} \beta_{2}\left(\pi_{2}\right)\right)$. If $p \in \operatorname{supp}\left(\pi_{1} \backslash \beta_{2}\left(\pi_{2}\right)\right)$, then we have $p \notin \beta_{2}(C)$ for all $C \in \pi_{2}$, and as $\beta_{1} \beta_{2}(C) \subseteq \beta_{2}(C), p \notin \beta_{1} \beta_{2}(C)$, hence $p \in \operatorname{supp}\left(\pi_{0} \backslash \beta_{1} \beta_{2}\left(\pi_{2}\right)\right)$; thus (b) $\operatorname{supp}\left(\pi_{1} \backslash \beta_{2}\left(\pi_{2}\right)\right) \subseteq \operatorname{supp}\left(\pi_{0} \backslash \beta_{1} \beta_{2}\left(\pi_{2}\right)\right)$. Now suppose that $p \in \operatorname{supp}\left(\pi_{0} \backslash \beta_{1} \beta_{2}\left(\pi_{2}\right)\right)$ and $p \notin \operatorname{supp}\left(\pi_{0} \backslash \beta_{1}\left(\pi_{1}\right)\right)$, so $p \notin \beta_{1} \beta_{2}(C)$ for all $C \in \pi_{2}$, but there exists $B \in \pi_{1}$ such that $p \in \beta_{1}(B)$; then $B \neq \beta_{2}(C)$ for all $C \in \pi_{2}$; as $\beta_{1}(B) \subseteq B$, we get $p \in B$, so $p \notin \beta_{2}(C)$ for all $C \in \pi_{2}$, hence $p \in \operatorname{supp}\left(\pi_{1} \backslash \beta_{2}\left(\pi_{2}\right)\right)$; thus (c) $\operatorname{supp}\left(\pi_{0} \backslash \beta_{1} \beta_{2}\left(\pi_{2}\right)\right) \subseteq \operatorname{supp}\left(\pi_{0} \backslash \beta_{1}\left(\pi_{1}\right)\right) \cup \operatorname{supp}\left(\pi_{1} \backslash \beta_{2}\left(\pi_{2}\right)\right)$. From the three inclusions $(\mathrm{a}, \mathrm{b}, \mathrm{c})$, the equality (17) follows.

Let $p \in \operatorname{supp}\left(\pi_{0} \backslash \beta_{1} \beta_{2}\left(\pi_{2}\right)\right)$. If $p \in \operatorname{supp}\left(\pi_{0} \backslash \beta_{1}\left(\pi_{1}\right)\right)$ and $p \notin \operatorname{supp}\left(\pi_{1} \backslash \beta_{2}\left(\pi_{2}\right)\right)$, there is some $C \in \pi_{2}$ such that $p \in \beta_{2}(C)$ but $p \notin \beta_{1} \beta_{2}(C)$; then (14) applied to $\beta_{1}$ gives $\alpha_{1}(p)=\beta_{1} \beta_{2}(C)$; now $p \in C$, so (14) applied to $\beta_{1} \beta_{2}$ gives $\alpha_{12}(p)=\beta_{1} \beta_{2}(C)$. Thus $\alpha_{12}(p)=\alpha_{1}(p)$ and item 1 holds. If $p \in \operatorname{supp}\left(\pi_{1} \backslash \beta_{2}\left(\pi_{2}\right)\right)$, there is some $C \in \pi_{2}$ such that $p \in C$ but $p \notin \beta_{2}(C)$, so $p \notin \beta_{1} \beta_{2}(C)$; then (14) applied to $\beta_{1} \beta_{2}$ gives $\alpha_{12}(p)=\beta_{1} \beta_{2}(C)$, while (14) applied to $\beta_{2}$ gives $\alpha_{2}(p)=\beta_{2}(C)$, hence $\beta_{1} \alpha_{2}(p)=\beta_{1} \beta_{2}(C)$. Thus $\alpha_{12}(p)=\beta_{1} \alpha_{2}(p)$ and item 2 holds.

Note that in case 2, the result does not depend on whether $p$ belongs to a block of $\pi_{0}$ that is apportioned or one that is enlarged, i.e., whether $p \in$ or $p \notin$ $\operatorname{supp}\left(\pi_{0} \backslash \beta_{1}\left(\pi_{1}\right)\right)$, see Figure 9 .

Now we complement Proposition 7 by showing that when the apportioning index is $>1$, the apportioning can be decomposed into two successive apportionings:

Lemma 8 Let $\pi_{0}, \pi_{2} \in \Pi^{*}(E) \backslash\{\emptyset\}$, and let $c, d$ be two integers $>0$. Suppose that $\beta$ apportions $\pi_{0}$ into $\pi_{2}$, with $I\left(\pi_{0}, \pi_{2}\right)=c+d$. Then there exists $\pi_{1} \in \Pi^{*}(E) \backslash\{\varnothing\}$ and two maps $\beta_{c}: \pi_{1} \rightarrow \pi_{0}$ and $\beta_{d}: \pi_{2} \rightarrow \pi_{1}$ such that $\beta_{c}$ apportions $\pi_{0}$ into $\pi_{1}, \beta_{d}$ apportions $\pi_{1}$ into $\pi_{2}, \beta=\beta_{c} \beta_{d}, I\left(\pi_{0}, \pi_{1}\right)=c$ and $I\left(\pi_{1}, \pi_{2}\right)=d$.

Proof Since $\beta\left(\pi_{2}\right) \subseteq \pi_{0}$ and $\left|\pi_{0} \backslash \beta\left(\pi_{2}\right)\right|=I\left(\pi_{0}, \pi_{2}\right)=c+d$, we can partition $\pi_{0} \backslash \beta\left(\pi_{2}\right)$ into two partial partitions $\pi_{c}$ and $\pi_{d}$ with $\left|\pi_{c}\right|=c$ and $\left|\pi_{d}\right|=d$; thus $\pi_{0}=\beta\left(\pi_{2}\right) \cup \pi_{c} \cup \pi_{d}$. Let $V=\operatorname{supp}\left(\beta\left(\pi_{2}\right)\right)$ and $W=\operatorname{supp}\left(\beta\left(\pi_{2}\right) \cup \pi_{c}\right)=\operatorname{supp}\left(\beta\left(\pi_{2}\right)\right) \cup$ 


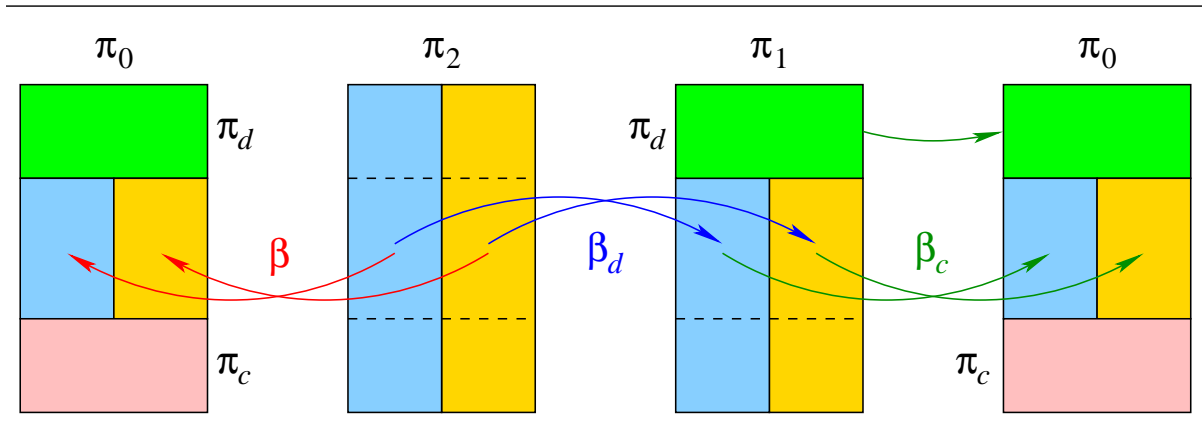

Fig. 10 From left to right: $\pi_{0}$, then $\pi_{2}$, with $\beta$ apportioning $\pi_{0}$ into $\pi_{2}$ and $\pi_{0} \backslash \beta\left(\pi_{2}\right)=\pi_{c} \cup \pi_{d}$ (here $c=d=1$ ); next $\pi_{1}$, with $\beta_{d}$ apportioning $\pi_{1}$ into $\pi_{2}$ and $\pi_{1} \backslash \beta_{d}\left(\pi_{2}\right)=\pi_{d}$, and finally $\pi_{0}$, with $\beta_{c}$ apportioning $\pi_{0}$ into $\pi_{1}$ and $\pi_{0} \backslash \beta_{c}\left(\pi_{1}\right)=\pi_{c}$. We have $\beta=\beta_{c} \beta_{d}$.

$\operatorname{supp}\left(\pi_{c}\right)=V \cup \operatorname{supp}\left(\pi_{c}\right) ;$ thus $V \subseteq W, W \cap \operatorname{supp}\left(\pi_{d}\right)=\emptyset$ and $W \cup \operatorname{supp}\left(\pi_{d}\right)=$ $\operatorname{supp}\left(\beta\left(\pi_{2}\right)\right) \cup \operatorname{supp}\left(\pi_{c}\right) \cup \operatorname{supp}\left(\pi_{d}\right)=\operatorname{supp}\left(\pi_{0}\right)$.

By (13), for any $C \in \pi_{2}$ we have $\beta(C)=C \cap V$; thus $\emptyset \subset \beta(C) \subseteq C \cap W \subseteq C$. Let $\pi^{*}=\left\{C \cap W \mid C \in \pi_{2}\right\}$, then $\pi^{*}$ is a partial partition and

$$
\operatorname{supp}\left(\pi^{*}\right)=\bigcup_{C \in \pi_{2}}(C \cap W)=\left(\bigcup_{C \in \pi_{2}} C\right) \cap W=\operatorname{supp}\left(\pi_{2}\right) \cap W=W .
$$

Let $\pi_{1}=\pi^{*} \cup \pi_{d}$; as $W \cap \operatorname{supp}\left(\pi_{d}\right)=\emptyset, \pi_{1} \in \Pi^{*}(E)$ and $\operatorname{supp}\left(\pi_{1}\right)=W \cup$ $\operatorname{supp}\left(\pi_{d}\right)=\operatorname{supp}\left(\pi_{0}\right)$. Define $\beta_{d}: \pi_{2} \rightarrow \pi_{1}: C \mapsto C \cap W$; then $\beta_{d}$ apportions $\pi_{1}$ into $\pi_{2}$ and $\pi_{1} \backslash \beta_{d}\left(\pi_{2}\right)=\pi_{1} \backslash \pi^{*}=\pi_{d}$, so $I\left(\pi_{1}, \pi_{2}\right)=\left|\pi_{d}\right|=d$. Define $\beta_{c}: \pi_{1} \rightarrow \pi_{0}$ by $\beta_{c}(B)=B$ for $B \in \pi_{d}$, and $\beta_{c}(B)=B \cap V$ for $B \in \pi^{*}$; in other words, for $C \in \pi_{2}$ we have $\beta_{c}(C \cap W)=C \cap V=\beta(C)$. Thus $\beta_{c}\left(\pi_{d}\right)=\pi_{d}$ and $\beta_{c}\left(\pi^{*}\right)=\beta\left(\pi_{2}\right)$, so $\beta_{c}\left(\pi_{1}\right)=\beta_{c}\left(\pi^{*}\right) \cup \beta_{c}\left(\pi_{d}\right)=\beta\left(\pi_{2}\right) \cup \pi_{d}$. Then $\beta_{c}$ apportions $\pi_{0}$ into $\pi_{1}$ and $\pi_{0} \backslash \beta_{c}\left(\pi_{1}\right)=\pi_{0} \backslash\left(\beta\left(\pi_{2}\right) \cup \pi_{d}\right)=\pi_{c}$, hence $I\left(\pi_{0}, \pi_{1}\right)=\left|\pi_{c}\right|=c$. Now for all $C \in \pi_{2}$, $\beta_{c}\left(\beta_{d}(C)\right)=\beta_{c}(C \cap W)=C \cap V=\beta(C)$, hence $\beta=\beta_{c} \beta_{d}$. See Figure 10 .

We now define the covering relation corresponding to the apportioning order $\stackrel{\text { a }}{\leq}$. For $\pi_{1}, \pi_{2} \in \Pi^{*}(E) \backslash\{\varnothing\}$, we write $\pi_{1} \stackrel{\text { a }}{\prec} \pi_{2}$ and say that $\pi_{2}$ a-covers $\pi_{1}$, if $\pi_{2}$ is obtained by apportioning a single block of $\pi_{1}$ among remaining blocks, that is, $\pi_{1} \stackrel{\text { a }}{<} \pi_{2}$ and $I\left(\pi_{1}, \pi_{2}\right)=1$. In other words:

$$
\pi_{1} \stackrel{\mathrm{a}}{\prec} \pi_{2} \Longleftrightarrow\left[\begin{array}{l}
\operatorname{supp}\left(\pi_{1}\right)=\operatorname{supp}\left(\pi_{2}\right) \&\left|\pi_{1}\right| \geq 2 \\
\& \exists A \in \pi_{1}, \pi_{1} \backslash\{A\} \stackrel{\mathrm{i}}{<} \pi_{2}
\end{array}\right] .
$$

We give the form of (18) with the map $\alpha$ of Lemma 4 :

$$
\pi_{1} \stackrel{\mathrm{a}}{\prec} \pi_{2} \Longleftrightarrow\left[\begin{array}{l}
\left|\pi_{1}\right| \geq 2 \& \exists A \in \pi_{1} \& \exists \alpha: A \rightarrow \pi_{1} \backslash\{A\}, \\
\pi_{2}=\left\{B \cup \alpha^{-1}(B) \mid B \in \pi_{1} \backslash\{A\}\right\}
\end{array}\right] .
$$

We can now give the main properties of the apportioning order:

Theorem 9 Apportioning $\stackrel{a}{\leq}$ is a partial order relation on $\Pi^{*}(E)$; it is included in the building order and it contains the merging order: for any $\pi_{1}, \pi_{2} \in \Pi^{*}(E), \pi_{1} \stackrel{\mathrm{m}}{\leq} \pi_{2} \Rightarrow$ $\pi_{1} \stackrel{\mathrm{a}}{\leq} \pi_{2} \Rightarrow \pi_{1} \Subset \pi_{2}$. 
The poset $\left(\Pi^{*}(E), \stackrel{\mathrm{a}}{\leq}\right)$ is the disjoint union of the posets $(\Pi(A), \Subset)$ for all $A \in$ $\mathcal{P}(E)$, where for distinct $A, A^{\prime} \in \mathcal{P}(E)$, elements of $\Pi(A)$ and $\Pi\left(A^{\prime}\right)$ are mutually incomparable. The maximal and minimal elements are all $\mathbf{1}_{A}$ and $\mathbf{0}_{A}$ respectively, for $A \in \mathcal{P}(E)$; every $\pi \in \Pi^{*}(E)$ majorates a unique minimal element, namely $\mathbf{0}_{\operatorname{supp}(\pi)}$. The covering relation is $\stackrel{\mathrm{a}}{\prec}$.

Let $E$ be finite. Then $\left(\Pi^{*}(E), \stackrel{\text { a }}{\leq}\right)$ is graded by $h_{m}$, that is, for any $\pi_{1}, \pi_{2} \in \Pi^{*}(E)$ we have

$$
\begin{aligned}
& \pi_{1} \stackrel{\mathrm{a}}{<} \pi_{2} \Longrightarrow h_{m}\left(\pi_{1}\right)<h_{m}\left(\pi_{2}\right), \\
& \pi_{1} \stackrel{\mathrm{a}}{\prec} \pi_{2} \Longrightarrow h_{m}\left(\pi_{2}\right)=h_{m}\left(\pi_{1}\right)+1 .
\end{aligned}
$$

For $\pi \in \Pi^{*}(E)$, the height of $\pi$ w.r.t. $\mathbf{0}_{\operatorname{supp}(\pi)}$ is $h_{m}(\pi)$. For $\pi_{1}, \pi_{2} \in \Pi^{*}(E)$ such that $\pi_{1} \stackrel{\mathrm{a}}{\leq} \pi_{2}$, we have $I\left(\pi_{1}, \pi_{2}\right)=h_{m}\left(\pi_{2}\right)-h_{m}\left(\pi_{1}\right)$.

Proof By $(15), \stackrel{\text { a }}{\leq}$ is the intersection of the building order $\Subset$ (a partial order by Proposition 2) and of the quasi-order given by support equality; it is thus a partial order relation included in $\Subset$. The merging order $\stackrel{m}{\leq}$ is included in the building order (by Proposition 2) and in the support equality, cf. (1); hence $\stackrel{m}{\leq}$ is included in the intersection of the two, namely the apportioning order $\stackrel{\text { a }}{\leq}$.

Obviously $\Pi^{*}(E)$ is the disjoint union of the $\Pi(A)$ for all $A \in \mathcal{P}(E)$, and $\pi_{1} \stackrel{\text { a }}{\leq} \pi_{2}$ means that for $A=\operatorname{supp}\left(\pi_{1}\right)=\operatorname{supp}\left(\pi_{2}\right)$, we have $A \in \mathcal{P}(E)$ with $\pi_{1}, \pi_{2} \in \Pi(A)$ and $\pi_{1} \Subset \pi_{2}$. For $A \in \mathcal{P}(E)$ and $\pi \in \Pi(A)$, we have $\mathbf{0}_{A} \Subset \pi \Subset \mathbf{1}_{A}$ with $\operatorname{supp}\left(\mathbf{0}_{A}\right)=$ $\operatorname{supp}(\pi)=\operatorname{supp}\left(\mathbf{1}_{A}\right)$, thus $\mathbf{0}_{A}$ and $\mathbf{1}_{A}$ are the least and greatest elements in $\Pi(A)$, so they are respectively minimal and maximal elements in $\Pi^{*}(E)$, and all minimal and maximal elements in $\Pi^{*}(E)$ are of this form. For $\pi \in \Pi^{*}(E)$, the unique minimal element majorated by $\pi$ is $\mathbf{0}_{A}$ for $A=\operatorname{supp}(\pi)$.

Let $\pi_{0}, \pi_{2} \in \Pi^{*}(E)$ such that $\pi_{0} \stackrel{\text { a }}{<} \pi_{2}$. Then $\pi_{0}, \pi_{2} \in \Pi^{*}(E) \backslash\{\varnothing\}$. By Lemma 6 , $I\left(\pi_{0}, \pi_{2}\right) \geq 1$. If $\pi_{2}$ does not cover $\pi_{0}$, then there is $\pi_{1} \in \Pi^{*}(E) \backslash\{\varnothing\}$ such that $\pi_{0} \stackrel{\text { a }}{<} \pi_{1} \stackrel{\text { a }}{<} \pi_{2}$; by Lemma $6, I\left(\pi_{0}, \pi_{1}\right) \geq 1$ and $I\left(\pi_{1}, \pi_{2}\right) \geq 1$, and then Proposition 7 gives $I\left(\pi_{0}, \pi_{2}\right)=I\left(\pi_{0}, \pi_{1}\right)+I\left(\pi_{1}, \pi_{2}\right) \geq 2$. Conversely, if $I\left(\pi_{0}, \pi_{2}\right) \geq 2$, by Lemma 8 there is $\pi_{1} \in \Pi^{*}(E) \backslash\{\emptyset\}$ such that $\pi_{0} \stackrel{\text { a }}{\leq} \pi_{1} \stackrel{\text { a }}{\leq} \pi_{2}, I\left(\pi_{0}, \pi_{1}\right)=1$ and $I\left(\pi_{1}, \pi_{2}\right)=I\left(\pi_{0}, \pi_{2}\right)-1 \geq 1$; by Lemma $6, \pi_{0} \stackrel{\text { a }}{<} \pi_{1} \stackrel{\text { a }}{<} \pi_{2}$, thus $\pi_{2}$ does not cover $\pi_{1}$. We have thus shown that $\pi_{2}$ does not cover $\pi_{1}$ if and only if $I\left(\pi_{0}, \pi_{2}\right) \geq 2$, therefore $\pi_{2}$ covers $\pi_{1}$ if and only if $I\left(\pi_{0}, \pi_{2}\right)=1$, that is, $\pi_{1} \stackrel{a}{\prec} \pi_{2}$.

Let $E$ be finite and $\pi_{1}, \pi_{2} \in \Pi^{*}(E)$ with $\pi_{1} \stackrel{\text { a }}{\leq} \pi_{2}$. Since $\left|\operatorname{supp}\left(\pi_{1}\right)\right|=\left|\operatorname{supp}\left(\pi_{2}\right)\right|$, by (8) we get

$$
h_{m}\left(\pi_{2}\right)-h_{m}\left(\pi_{1}\right)=\left(\left|\operatorname{supp}\left(\pi_{2}\right)\right|-\left|\pi_{2}\right|\right)-\left(\left|\operatorname{supp}\left(\pi_{1}\right)\right|-\left|\pi_{1}\right|\right)=\left|\pi_{1}\right|-\left|\pi_{2}\right| .
$$

If $\pi_{1}=\pi_{2}=\varnothing$, then $I\left(\pi_{1}, \pi_{2}\right)=I(\varnothing, \varnothing)=0=h_{m}(\varnothing)-h_{m}(\varnothing)$; otherwise, $\pi_{1}, \pi_{2} \in \Pi^{*}(E) \backslash\{\emptyset\}$, and given $\beta$ that apportions $\pi_{1}$ into $\pi_{2}$,

$$
I\left(\pi_{1}, \pi_{2}\right)=\left|\pi_{1} \backslash \beta\left(\pi_{2}\right)\right|=\left|\pi_{1}\right|-\left|\beta\left(\pi_{2}\right)\right|=\left|\pi_{1}\right|-\left|\pi_{2}\right|=h_{m}\left(\pi_{2}\right)-h_{m}\left(\pi_{1}\right) .
$$

If $\pi_{1} \stackrel{\mathrm{a}}{<} \pi_{2}$, then $I\left(\pi_{1}, \pi_{2}\right)>0$, hence $h_{m}\left(\pi_{2}\right)-h_{m}\left(\pi_{1}\right)>0$. If $\pi_{1} \stackrel{\mathrm{a}}{\prec} \pi_{2}$, then $I\left(\pi_{1}, \pi_{2}\right)=1$, hence $h_{m}\left(\pi_{2}\right)-h_{m}\left(\pi_{1}\right)=1$. Therefore $\left(\Pi^{*}(E), \stackrel{\text { a }}{\leq}\right)$ is graded by $h_{m}$. For $\pi \in \Pi^{*}(E), h_{m}\left(\mathbf{0}_{\operatorname{supp}(\pi)}\right)=0$, cf. Table 1 , so the height of $\pi$ w.r.t. $\mathbf{0}_{\operatorname{supp}(\pi)}$ is $h_{m}(\pi)-h_{m}\left(\mathbf{0}_{\operatorname{supp}(\pi)}\right)=h_{m}(\pi)$. 
We note that the statement of Theorem 9 is similar to the corresponding one for the merging order (cf. Table 1, see also Theorem 7 of [16] for more details), where we only replace $\stackrel{\mathrm{m}}{\leq}$ and $\stackrel{\mathrm{m}}{\prec}$ by $\stackrel{\text { a }}{\leq}$ and $\stackrel{\mathrm{a}}{\prec}$.

The apportioning order does not constitute a lattice. Indeed, let $\{A, B, C, D\} \in$ $\Pi^{*}(E)$, i.e., $A, B, C, D$ are mutually disjoint non-empty subsets of $E$. Then each of the 3 partial partitions obtained by merging $A, B, C, D$ by pairs will cover each of the 6 partial partitions obtained by merging just two of $A, B, C, D$, that is:

$$
\left.\begin{array}{l}
\{A \cup B, C, D\},\{C \cup D, A, B\} \\
\{A \cup C, B, D\},\{B \cup D, A, C\} \\
\{A \cup D, B, C\},\{B \cup C, A, D\}
\end{array}\right\} \stackrel{\prec}{\prec}\left\{\begin{array}{l}
\{A \cup B, C \cup D\}, \\
\{A \cup C, B \cup D\} \\
\{A \cup D, B \cup C\}
\end{array},\right.
$$

This is impossible in a lattice, because each of the 3 greater ones is a minimal upper bound of the 6 lower ones, and each of the 6 lower ones is a maximal lower bound of the 3 greater ones.

Now consider the following 4 partial partitions built from $A, B, C, D$ :

$$
\begin{array}{rlrl}
\pi^{0} & =\{A, B, C, D\}, & & \pi^{1}=\{A \cup B, C, D\}, \\
\pi^{2} & =\{A \cup C, B \cup D\}, & \pi^{3}=\{A \cup B \cup C \cup D\} .
\end{array}
$$

Then $\pi^{0} \stackrel{\mathrm{m}}{\prec} \pi^{1} \stackrel{\mathrm{a}}{\prec} \pi^{2} \stackrel{\mathrm{m}}{\prec} \pi^{3}, \pi^{0} \stackrel{\mathrm{m}}{<} \pi^{2}, \pi^{1} \stackrel{\mathrm{m}}{<} \pi^{3}$ and $\pi^{0} \stackrel{\mathrm{m}}{<} \pi^{3}$. This shows that we cannot separate apportioning from merging in that order.

In Property 5 of [16] we gave an example showing that (when $|E| \geq 5$ ) the intersection of the apportioning order and of the singularity relation is not transitive. We recall it here. Let $\{J, K, L, M, N\} \in \Pi^{*}(E)$, i.e., $J, K, L, M, N$ are mutually disjoint non-empty subsets of $E$, and let

$$
\pi_{0}=\{J, K, L \cup M, N\}, \quad \pi_{1}=\{J, K \cup L, M \cup N\}, \quad \pi_{2}=\{J \cup K, L \cup M \cup N\} .
$$

Then: $\pi_{0} \stackrel{\mathrm{a}}{\prec} \pi_{1} \stackrel{\mathrm{a}}{\prec} \pi_{2}, \pi_{0} \stackrel{\mathrm{m}}{\gtrless} \pi_{1} \stackrel{\mathrm{m}}{\gtrless} \pi_{2}, \pi_{0} \Leftarrow \pi_{1} \Leftarrow \pi_{2}, \pi_{0} \stackrel{\mathrm{m}}{<} \pi_{2}$ and $\pi_{0} \notin \pi_{2}$. In other words, $\pi_{1}$ (resp., $\pi_{2}$ ) is obtained by apportioning one block of $\pi_{0}$ (resp., $\pi_{1}$ ) to two other blocks, and not by merging blocks, thus every block of $\pi_{1}$ (resp., $\pi_{2}$ ) contains exactly one block of $\pi_{0}$ (resp., $\pi_{1}$ ); however, each of the two blocks of $\pi_{2}$ is obtained by merging two blocks of $\pi_{0}$.

The apportioning order was initially defined by the succession of two operations: first removing some block, next inflating the remaining blocks until the initial support of the partial partition is covered. One can admit the possibility of continuing to inflate these remaining blocks beyond the recovery of the initial support; this leads to the apportioning-inflating order $\leq$ ai that we define mathematically as the intersection of the building order and the support inclusion relation:

$$
\forall \pi_{1}, \pi_{2} \in \Pi^{*}(E), \quad \pi_{1} \stackrel{\text { ai }}{\leq} \pi_{2} \Longleftrightarrow\left[\pi_{1} \Subset \pi_{2} \& \operatorname{supp}\left(\pi_{1}\right) \subseteq \operatorname{supp}\left(\pi_{2}\right)\right] .
$$

Before giving an interpretation of it in terms of a map $\beta$ as in Lemma 4, we first give its main order-theoretic properties:

Theorem 10 Apportioning-inflating is a partial order relation on $\Pi^{*}(E)$; it is included in the building order and it contains the apportioning and merging-inflating orders: for any $\pi_{1}, \pi_{2} \in \Pi^{*}(E), \pi_{1} \stackrel{\text { a }}{\leq} \pi_{2} \Rightarrow \pi_{1} \stackrel{\text { ai }}{\leq} \pi_{2}, \pi_{1} \stackrel{\text { mi }}{\leq} \pi_{2} \Rightarrow \pi_{1} \stackrel{\text { ai }}{\leq} \pi_{2}$ and 
$\pi_{1} \stackrel{\text { ai }}{\leq} \pi_{2} \Longrightarrow \pi_{1} \Subset \pi_{2}$. It is generated by composing apportioning and inflating in any order:

$$
\begin{aligned}
\pi_{1} \stackrel{\text { ai }}{\leq} \pi_{2} & \Longleftrightarrow\left(\exists \pi_{3} \in \Pi^{*}(E), \pi_{1} \stackrel{\text { a }}{\leq} \pi_{3} \stackrel{\text { i }}{\leq} \pi_{2}\right) \\
& \Longleftrightarrow\left(\exists \pi_{4} \in \Pi^{*}(E), \pi_{1} \stackrel{\mathrm{i}}{\leq} \pi_{4} \stackrel{\mathrm{a}}{\leq} \pi_{2}\right) .
\end{aligned}
$$

$\varnothing$ is isolated. In $\Pi^{*}(E) \backslash\{\varnothing\}$, the greatest element is $\mathbf{1}_{E}$ and the minimal elements are all $\mathbf{0}_{A}$ for $A \in \mathcal{P}(E) \backslash\{\emptyset\}$. Given $\pi \in \Pi^{*}(E)$, the minimal elements majorated by $\pi$ are the $\mathbf{0}_{A}$ for all crossings $A$ of $\pi$ (for $\pi=\emptyset, A=\emptyset$ and $\mathbf{0}_{A}=\varnothing$ ). The covering relation is $\stackrel{\mathrm{a}}{\prec} \cup \stackrel{\mathrm{i}}{\prec}$.

Let $E$ be finite. Then $\left(\Pi^{*}(E), \stackrel{\text { ai }}{\leq}\right)$ is graded by $\left(-h_{c}, h_{s}\right)$ for $(\stackrel{\text { a }}{\prec, ~} \underset{\gtrless}{\prec})$, that is, for any $\pi_{1}, \pi_{2} \in \Pi^{*}(E)$ we have

$$
\begin{aligned}
\pi_{1} \stackrel{\text { ai }}{<} \pi_{2} & \Longrightarrow\left[\begin{array}{l}
h_{s}\left(\pi_{1}\right) \leq h_{s}\left(\pi_{2}\right) \& \\
h_{c}\left(\pi_{1}\right) \geq h_{c}\left(\pi_{2}\right) \& \\
h_{m}\left(\pi_{1}\right)<h_{m}\left(\pi_{2}\right)
\end{array}\right], \\
\pi_{1} \stackrel{\text { a }}{\prec} \pi_{2} & \Longrightarrow\left[\begin{array}{l}
h_{s}\left(\pi_{2}\right)=h_{s}\left(\pi_{1}\right) \& \\
h_{c}\left(\pi_{2}\right)=h_{c}\left(\pi_{1}\right)-1
\end{array}\right], \\
\pi_{1} \stackrel{\mathrm{i}}{\prec} \pi_{2} & \Longrightarrow\left[\begin{array}{l}
h_{c}\left(\pi_{2}\right)=h_{c}\left(\pi_{1}\right) \& \\
h_{s}\left(\pi_{2}\right)=h_{s}\left(\pi_{1}\right)+1
\end{array}\right] .
\end{aligned}
$$

In particular, $\left(\Pi^{*}(E), \stackrel{\text { ai }}{\leq}\right)$ is graded by $h_{m}=h_{s}-h_{c}$. For $\pi \in \Pi^{*}(E)$ and a crossing $A$ of $\pi$, the height of $\pi$ w.r.t. $\mathbf{0}_{A}$ is $h_{m}(\pi)$.

Proof Being the intersection of the partial order $\Subset$ (by Proposition 2) and of the quasi-order given by support inclusion, $\stackrel{\text { ai }}{\leq}$ is a partial order relation included in $\Subset$. Since support equality is a particular case of support inclusion, $\stackrel{\text { ai }}{\leq}$ contains $\stackrel{\text { a }}{\leq}$. For $\pi_{1} \stackrel{\text { mi }}{\leq} \pi_{2}$, by (3) we have $\pi_{1} \Subset \pi_{2}$ and $\pi_{1} \leq \pi_{2}$, so $\operatorname{supp}\left(\pi_{1}\right) \subseteq \operatorname{supp}\left(\pi_{2}\right)$, hence $\pi_{1} \stackrel{\text { ai }}{\leq} \pi_{2}$.

If $\pi_{1} \stackrel{\text { a }}{\leq} \pi_{3} \stackrel{\text { i }}{\leq} \pi_{2}$, then $\pi_{1} \stackrel{\text { ai }}{\leq} \pi_{3} \stackrel{\text { ai }}{\leq} \pi_{2}$, and if $\pi_{1} \stackrel{\text { i }}{\leq} \pi_{4} \stackrel{\text { a }}{\leq} \pi_{2}$, then $\pi_{1} \stackrel{\text { ai }}{\leq} \pi_{4} \stackrel{\text { ai }}{\leq} \pi_{2}$, thus $\pi_{1} \leq \pi_{2}$ in both cases. Suppose now that $\pi_{1} \stackrel{\text { ai }}{\leq} \pi_{2}$. We will construct $\pi_{3}, \pi_{4} \in \Pi^{*}(E)$ such that $\pi_{1} \stackrel{\text { a }}{\leq} \pi_{3} \stackrel{\text { i }}{\leq} \pi_{2}$ and $\pi_{1} \stackrel{\text { i }}{\leq} \pi_{4} \stackrel{\text { a }}{\leq} \pi_{2}$; this is illustrated in Figure 11 .

Let $V=\operatorname{supp}\left(\pi_{1}\right)$. Since $\pi_{1} \Subset \pi_{2}$, every block $C \in \pi_{2}$ contains a block $B \in \pi_{1}$, and as $B \subseteq V$, we have $\emptyset \subset B \subseteq C \cap V \subseteq C$, thus $C \cap V \neq \emptyset$. Hence $\pi_{3}=\{C \cap V \mid$ $\left.C \in \pi_{2}\right\}$ is a partial partition, and $\pi_{1} \Subset \pi_{3}$, since for every $C \in \pi_{2}, C \cap V$ contains a block $B$ of $\pi_{1}$ (included in $C$ ); we have

$$
\operatorname{supp}\left(\pi_{3}\right)=\bigcup_{C \in \pi_{2}}(C \cap V)=\operatorname{supp}\left(\pi_{2}\right) \cap V=V,
$$

hence $\pi_{1} \stackrel{a}{\leq} \pi_{3}$. Also for two distinct $C, D \in \pi_{2}$, since $C$ and $D$ are disjoint, $C \cap V \nsubseteq$ $D$; thus for every $C \in \pi_{2}, C \cap V$ is the unique block of $\pi_{3}$ included in $\pi_{2}$ and $C$ is the unique block of $\pi_{2}$ containing $\pi_{3}$, thus $\pi_{3} \stackrel{i}{\leq} \pi_{2}$.

By Proposition 3, there is some $\pi \subseteq \pi_{1}$ such that $\pi \stackrel{i}{\leq} \pi_{2}$, so there is a bijection $f: \pi_{2} \rightarrow \pi$ such that for any $C \in \pi_{2}, f(C) \subseteq C$ (but $f(C) \nsubseteq D$ for any other 


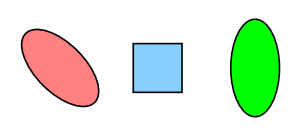

$\pi_{1}$

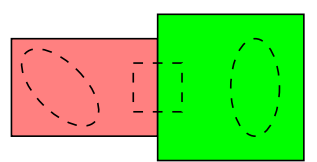

$\pi_{2}$

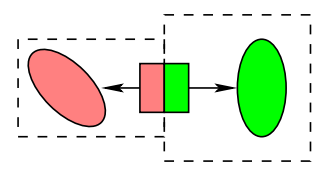

apportion $\pi_{3}$

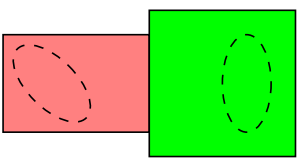

inflate

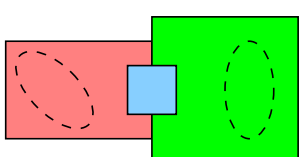

inflate $\pi_{4}$

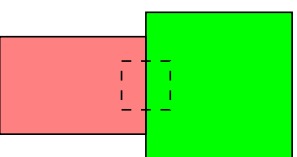

apportion

Fig. 11 Illustration of Theorem 10: from $\pi_{1} \stackrel{\text { ai }}{\leq} \pi_{2}$ we get $\pi_{1} \stackrel{\text { a }}{\leq} \pi_{3} \stackrel{\text { i }}{\leq} \pi_{2}$ and $\pi_{1} \stackrel{i}{\leq} \pi_{4} \stackrel{\text { a }}{\leq} \pi_{2}$.

$\left.D \in \pi_{2}\right)$. Let $\pi_{0}=\pi_{1} \backslash \pi$ and for every $C \in \pi_{2}$, let $g(C)=C \backslash \operatorname{supp}\left(\pi_{0}\right)$; then $f(C) \in \pi, f(C) \cap \operatorname{supp}\left(\pi_{0}\right)=\emptyset$, thus $\emptyset \subset f(C) \subseteq C \backslash \operatorname{supp}\left(\pi_{0}\right)=g(C)$. Let $\pi^{*}=$ $\left\{g(C) \mid C \in \pi_{2}\right\}$; then $\pi^{*}$ is a partial partition and $\pi \stackrel{i}{\leq} \pi^{*}$, since for every $C \in \pi_{2}$, $f(C)$ is the only block of $\pi$ included in $g(C)$, and $g(C)$ is the only block of $\pi^{*}$ containing $f(C)$. Now

$$
\operatorname{supp}\left(\pi^{*}\right)=\bigcup_{C \in \pi_{2}} g(C)=\bigcup_{C \in \pi_{2}}\left(C \backslash \operatorname{supp}\left(\pi_{0}\right)\right)=\operatorname{supp}\left(\pi_{2}\right) \backslash \operatorname{supp}\left(\pi_{0}\right)
$$

Let $\pi_{4}=\pi_{0} \cup \pi^{*} ; \operatorname{since} \operatorname{supp}\left(\pi^{*}\right) \cap \operatorname{supp}\left(\pi_{0}\right)=\emptyset, \pi_{4}$ is a partial partition; as $\pi \stackrel{i}{\leq} \pi^{*}$ and trivially $\pi_{0} \stackrel{i}{\leq} \pi_{0}$, we deduce that $\pi_{1}=\pi_{0} \cup \pi \stackrel{i}{\leq} \pi_{0} \cup \pi^{*}=\pi_{4}$. Now for every $C \in \pi_{2}, C$ contains $g(C) \in \pi^{*} \subseteq \pi_{4}$, thus $\pi_{4} \Subset \pi_{2}$. But $\operatorname{supp}\left(\pi_{4}\right)=$ $\operatorname{supp}\left(\pi_{0}\right) \cup \operatorname{supp}\left(\pi^{*}\right)=\operatorname{supp}\left(\pi_{2}\right) ;$ hence $\pi_{4} \stackrel{\mathrm{a}}{\leq} \pi_{2}$.

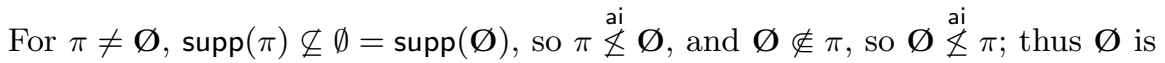
isolated. For $\pi \in \Pi^{*}(E) \backslash\{\varnothing\}, \pi \Subset \mathbf{1}_{E}$ and $\operatorname{supp}(\pi) \subseteq E=\operatorname{supp}\left(\mathbf{1}_{E}\right)$, so $\pi \stackrel{\text { ai }}{\leq} \mathbf{1}_{E}$; thus $\mathbf{1}_{E}$ is the greatest element in $\Pi^{*}(E) \backslash\{\emptyset\}$. Let $\pi \in \Pi^{*}(E)$ and $A \in \mathcal{P}(E)$ such that $\pi \stackrel{\text { ai }}{\leq} \mathbf{0}_{A}$; then $\operatorname{supp}(\pi) \subseteq \operatorname{supp}\left(\mathbf{0}_{A}\right)=A$ and for every $p \in A,\{p\}$ contains a block of $\pi$, that is, $\{p\} \in \pi$, thus $\mathbf{0}_{A} \subseteq \pi$; we deduce then that $\pi=\mathbf{0}_{A}$; therefore $\mathbf{0}_{A}$ is a minimal element in $\Pi^{*}(E) \backslash\{\bar{\varnothing}\}$. For $\pi \in \Pi^{*}(E)$ and $A \in \mathcal{P}(E)$, we have $\mathbf{0}_{A} \stackrel{\text { ai }}{\leq} \pi$ if and only $A=\operatorname{supp}\left(\mathbf{0}_{A}\right) \subseteq \operatorname{supp}(\pi)$ and every block $B \in \pi$ contains at least one block of $\mathbf{0}_{A}$, that is, $B \gamma A$; in other words $A$ is a crossing of $\pi$.

Let $\pi_{1} \stackrel{a}{\prec} \pi_{2} ;$ then $\operatorname{supp}\left(\pi_{1}\right)=\operatorname{supp}\left(\pi_{2}\right)$. If $\pi_{1} \stackrel{\text { ai }}{<} \pi \stackrel{\text { ai }}{<} \pi_{2}$, then $\pi_{1} \Subset \pi \Subset \pi_{2}$ and $\operatorname{supp}\left(\pi_{1}\right) \subseteq \operatorname{supp}(\pi) \subseteq \operatorname{supp}\left(\pi_{2}\right)$, thus $\operatorname{supp}\left(\pi_{1}\right)=\operatorname{supp}(\pi)=\operatorname{supp}\left(\pi_{2}\right)$, and we get $\pi_{1} \stackrel{\text { a }}{<} \pi \stackrel{\text { a }}{<} \pi_{2}$, which contradicts the fact that $\stackrel{a}{\prec}$ is the covering relation for $\stackrel{\text { a }}{\leq}$ (Theorem 9). Hence $\pi_{2}$ covers $\pi_{1}$ for $\stackrel{\text { ai }}{\leq}$. Now let $\pi_{1} \stackrel{i}{\prec} \pi_{2}$; then $\pi_{1} \stackrel{i}{\leq} \pi_{2}$. If $\pi_{1} \stackrel{\text { ai }}{<} \pi \stackrel{\text { ai }}{<} \pi_{2}$, then $\pi_{1} \Subset \pi \Subset \pi_{2}$, so (12) gives $\pi_{0} \stackrel{\text { i }}{<} \pi_{1} \stackrel{i}{<} \pi_{2}$, which contradicts the fact that $\stackrel{i}{\prec}$ is the covering relation for $\stackrel{i}{\leq}$ (Table 1 ). Hence $\pi_{2}$ covers $\pi_{1}$ for $\stackrel{\text { ai }}{\leq}$. 
Conversely, let $\pi_{2}$ cover $\pi_{1}$. We obtain $\pi_{1} \stackrel{\text { a }}{\leq} \pi_{3} \stackrel{i}{\leq} \pi_{2}$ as above; the three cases $\pi_{1} \stackrel{\mathrm{a}}{<} \pi_{3} \stackrel{\mathrm{i}}{<} \pi_{2}, \pi_{1}=\pi_{3} \stackrel{\mathrm{i}}{<} \pi \stackrel{\mathrm{i}}{<} \pi_{2}$ and $\pi_{1} \stackrel{\mathrm{a}}{<} \pi \stackrel{\mathrm{a}}{<} \pi_{3}=\pi_{2}$ contradict the covering of $\pi_{1}$ by $\pi_{2}$, so there remain only the cases where $\pi_{2}$ covers $\pi_{1}$ for $\stackrel{\text { a }}{\leq}$ or for $\stackrel{i}{\leq}$, that is, $\pi_{1} \stackrel{a}{\prec} \pi_{2}$ or $\pi_{1} \stackrel{i}{\prec} \pi_{2}$ (cf. Theorem 9 and Table 1 ). We have thus shown that the covering relation for $\stackrel{\text { ai }}{\leq}$ is $\stackrel{a}{\prec} \cup \stackrel{\text { i }}{\prec}$.

Let $E$ be finite. For $\pi_{1} \stackrel{\text { ai }}{\leq} \pi_{2}$, by (20) we have $\operatorname{supp}\left(\pi_{1}\right) \subseteq \operatorname{supp}\left(\pi_{2}\right)$, hence $h_{s}\left(\pi_{1}\right)=\left|\operatorname{supp}\left(\pi_{1}\right)\right| \leq\left|\operatorname{supp}\left(\pi_{2}\right)\right|=h_{s}\left(\pi_{2}\right)$; since $\pi_{1} \Subset \pi_{2}$, there is some $\pi \subseteq \pi_{1}$ such that $\pi \stackrel{i}{\leq} \pi_{2}$, so $h_{c}\left(\pi_{1}\right)=\left|\pi_{1}\right| \geq|\pi|=\left|\pi_{2}\right|=h_{c}\left(\pi_{2}\right)$; now $h_{m}=h_{s}-h_{c}$ by (8), so

$$
h_{m}\left(\pi_{2}\right)-h_{m}\left(\pi_{1}\right)=\left(h_{s}\left(\pi_{2}\right)-h_{s}\left(\pi_{1}\right)\right)+\left(h_{c}\left(\pi_{1}\right)-h_{c}\left(\pi_{2}\right)\right) \geq 0,
$$

with both terms $h_{s}\left(\pi_{2}\right)-h_{s}\left(\pi_{1}\right)$ and $h_{c}\left(\pi_{1}\right)-h_{c}\left(\pi_{2}\right)$ being $\geq 0$. If $h_{m}\left(\pi_{2}\right)=h_{m}\left(\pi_{1}\right)$, then $\operatorname{supp}\left(\pi_{2}\right)=h_{s}\left(\pi_{2}\right)=h_{s}\left(\pi_{1}\right)=\operatorname{supp}\left(\pi_{1}\right)$, and as $\operatorname{supp}\left(\pi_{1}\right) \subseteq \operatorname{supp}\left(\pi_{2}\right)$, we have $\operatorname{supp}\left(\pi_{1}\right)=\operatorname{supp}\left(\pi_{2}\right)$, hence $\pi_{1} \stackrel{\text { a }}{\leq} \pi_{2}$; now $\stackrel{\text { a }}{\leq}$ is graded by $h_{m}$, see Theorem 9 , so $h_{m}\left(\pi_{2}\right)=h_{m}\left(\pi_{1}\right)$ gives $\pi_{1}=\pi_{2}$. Therefore $\pi_{1} \stackrel{\text { ai }}{<} \pi_{2}$ implies that $h_{s}\left(\pi_{1}\right) \leq h_{s}\left(\pi_{2}\right)$, $h_{c}\left(\pi_{1}\right) \geq h_{c}\left(\pi_{2}\right)$ and $h_{m}\left(\pi_{1}\right)<h_{m}\left(\pi_{2}\right)$. Now $\pi_{1} \stackrel{a}{\prec} \pi_{2}$ gives $h_{s}\left(\pi_{2}\right)=\left|\operatorname{supp}\left(\pi_{2}\right)\right|=$ $\left|\operatorname{supp}\left(\pi_{1}\right)\right|=h_{s}\left(\pi_{1}\right)$ and $h_{c}\left(\pi_{2}\right)=\left|\pi_{2}\right|=\left|\pi_{1}\right|-1=h_{c}\left(\pi_{1}\right)-1$ by (18). Finally $\pi_{1} \stackrel{\mathrm{i}}{\prec} \pi_{2}$ gives $h_{s}\left(\pi_{2}\right)=\left|\operatorname{supp}\left(\pi_{2}\right)\right|=\left|\operatorname{supp}\left(\pi_{1}\right)\right|+1=h_{s}\left(\pi_{1}\right)+1$ and $h_{c}\left(\pi_{2}\right)=$ $\left|\pi_{2}\right|=\left|\pi_{1}\right|=h_{c}\left(\pi_{1}\right)$. Therefore $\left(\Pi^{*}(E), \stackrel{\text { ai }}{\leq}\right)$ is graded by $\left(-h_{c}, h_{s}\right)$ for $(\stackrel{\text { a }}{\prec}, \stackrel{\mathrm{i}}{\prec})$; by Proposition 1 , it is graded by $h_{m}=h_{s}-h_{c}$.

For a crossing $A$ of $\pi, h_{m}\left(\mathbf{0}_{A}\right)=0$, cf. Table 1 , so the height of $\pi$ w.r.t. $\mathbf{0}_{A}$ is $h_{m}(\pi)-h_{m}\left(\mathbf{0}_{A}\right)=h_{m}(\pi)$.

We note that the statement of Theorem 10 is similar to the corresponding one for the merging-inflating order (cf. Table 2, see also Theorem 12 of [16] for more details), where we only replace $\stackrel{m}{\leq}, \stackrel{\mathrm{mi}}{\leq}$ and $\stackrel{\mathrm{m}}{\prec}$ by $\stackrel{\mathrm{a}}{\leq}, \stackrel{\text { ai }}{\leq}$ and $\stackrel{\mathrm{a}}{\prec}$. Compare also the present Figure 11 with Figure 7 of [16]. Given the similarity of the statement of Theorem 9 with the corresponding one for the merging order, this strenghtens our suggestion that apportioning is a generalization of block merging.

We will now interpret the apportioning-inflating order in terms of functions defined on blocks, as we did in Lemma 4 for the apportioning order. Let $\pi_{1} \stackrel{\text { ai }}{\leq} \pi_{2}$. Since $\pi_{1} \Subset \pi_{2}$, there is a map $\beta: \pi_{2} \rightarrow \pi_{1}$ such that for every $C \in \pi_{2}, \beta(C) \subseteq C$. Let $W=\operatorname{supp}\left(\pi_{2}\right) \backslash \operatorname{supp}\left(\pi_{1}\right)$ and $\pi_{a}=\pi_{1} \cup \mathbf{1}_{W}$, that is, $\pi_{a}=\pi_{1}$ if $\operatorname{supp}\left(\pi_{1}\right)=\operatorname{supp}\left(\pi_{2}\right)$ and $\pi_{a}=\pi_{1} \cup\{W\}$ if $\operatorname{supp}\left(\pi_{1}\right) \subset \operatorname{supp}\left(\pi_{2}\right)$. Then $\pi_{a} \in \Pi^{*}(E), \operatorname{supp}\left(\pi_{a}\right)=\operatorname{supp}\left(\pi_{2}\right)$, hence $\pi_{a} \stackrel{\text { a }}{\leq} \pi_{2}$. Now we can consider $\beta$ as a map $\pi_{2} \rightarrow \pi_{a}$ that apportions $\pi_{a}$ into $\pi_{2}$, and by Lemma 4 we can define $\alpha$ : $\operatorname{supp}\left(\pi_{a} \backslash \beta\left(\pi_{2}\right)\right) \rightarrow \beta\left(\pi_{2}\right)$ according to (14): for $C \in \pi_{2}$ and $p \in \operatorname{supp}\left(\pi_{a} \backslash \beta\left(\pi_{2}\right)\right), \alpha(p)=\beta(C) \Leftrightarrow p \in C$. We partition $\operatorname{supp}\left(\pi_{a} \backslash \beta\left(\pi_{2}\right)\right)$ into $W=\operatorname{supp}\left(\pi_{a} \backslash \pi_{1}\right)$ and $\operatorname{supp}\left(\pi_{1} \backslash \beta\left(\pi_{2}\right)\right)$. Then the restriction $\alpha_{a}$ of $\alpha$ to supp $\left(\pi_{1} \backslash \beta\left(\pi_{2}\right)\right)$ represents the apportioning of blocks of $\pi_{1}$, while the restriction $\alpha_{i}$ of $\alpha$ to $W=\operatorname{supp}\left(\pi_{2}\right) \backslash \operatorname{supp}\left(\pi_{1}\right)$ describes the apportioning of $W$, in other words the inflation of the blocks, see Figure 12.

Let us give an alternative description of this construction. Let $V=\operatorname{supp}\left(\pi_{1}\right)$ and $\pi_{3}=\left\{C \cap V \mid C \in \pi_{2}\right\}$. From the proof of Theorem 10, $\pi_{3} \in \Pi^{*}(E)$ and $\pi_{1} \stackrel{\mathrm{a}}{\leq} \pi_{3} \stackrel{\mathrm{i}}{\leq} \pi_{2}$. Take $\pi_{a}=\pi_{1} \cup \mathbf{1}_{W}$ and $\pi_{b}=\pi_{3} \cup \mathbf{1}_{W}$; then $\pi_{a} \stackrel{\mathrm{a}}{\leq} \pi_{b} \stackrel{\mathrm{a}}{\leq} \pi_{2}$. 


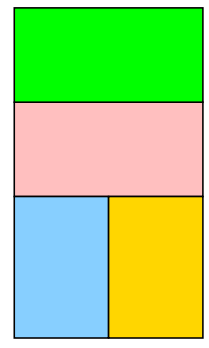

$\pi_{1}$

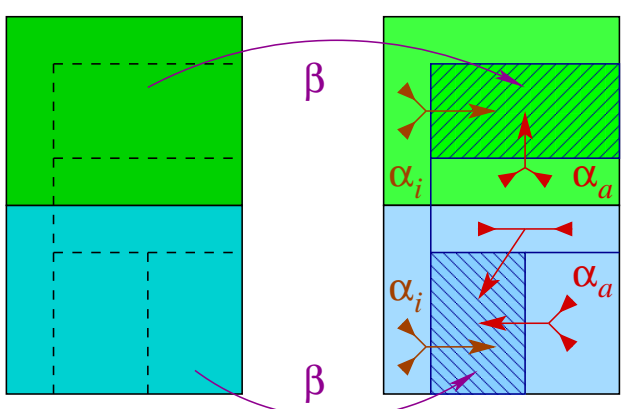

$\pi_{2}$

Fig. 12 Left: $\pi_{1}$. Middle: $\pi_{2}$; here $\pi_{1} \stackrel{\text { ai }}{\leq} \pi_{2}$. Right: one possible choice for $\beta$ (with the chosen block of $\pi_{1}$ shown hatched), and the resulting maps $\alpha_{a}$ and $\alpha_{i}$, apportioning $\pi_{1}$ and $\operatorname{supp}\left(\pi_{2}\right) \backslash$ $\operatorname{supp}\left(\pi_{1}\right)$ into $\pi_{2}$.

There is $\beta_{1}: \pi_{b} \rightarrow \pi_{a}$ apportioning $\pi_{a}$ into $\pi_{b}$, with the corresponding map $\alpha_{1}$ : $\operatorname{supp}\left(\pi_{a} \backslash \beta_{1}\left(\pi_{b}\right)\right) \rightarrow \beta_{1}\left(\pi_{b}\right)$; in fact only blocks of $\pi_{1}$ will be apportioned, $W$ will remain unchanged. There is $\beta_{2}: \pi_{2} \rightarrow \pi_{b}$ apportioning $\pi_{b}$ into $\pi_{2}$, with the corresponding map $\alpha_{2}: \operatorname{supp}\left(\pi_{b} \backslash \beta_{2}\left(\pi_{2}\right)\right) \rightarrow \beta_{2}\left(\pi_{2}\right)$; here $W$ is the only block that is apportioned. Then, following Proposition 7 and Figure 9, we will have $\beta=\beta_{1} \beta_{2}, \alpha_{a}=\alpha_{1}$ and $\alpha_{i}=\beta_{1} \alpha_{2}$. For $C \in \pi_{2}, C \cap V=\beta(C) \cup \alpha_{a}^{-1}(\beta(C))$ and $C=\beta(C) \cup \alpha_{a}^{-1}(\beta(C)) \cup \alpha_{i}^{-1}(\beta(C))$; also $\pi_{3}=\left\{\beta(C) \cup \alpha_{a}^{-1}(\beta(C)) \mid C \in \pi_{2}\right\}$.

\subsection{The extended order as a generalization of the standard order}

We now consider our third new order, the extended order; contrarily to the apportioning and apportioning-inflating orders, it is not included in the building order. We will see that it generalizes the standard order by allowing, on top of creating, merging and inflating blocks, apportioning. We will exhibit several analogies between the merging, merging-inflating and standard orders on the one hand, the apportioning, apportioning-inflating and extended orders on the other hand.

We define the extended order $\stackrel{\mathrm{e}}{\leq}$ as follows:

$$
\forall \pi_{1}, \pi_{2} \in \Pi^{*}(E), \quad \pi_{1} \stackrel{\mathrm{e}}{\leq} \pi_{2} \Longleftrightarrow\left[\begin{array}{l}
\pi_{1} \Subset \pi_{2} \cap \mathcal{P}\left(\operatorname{supp}\left(\pi_{1}\right)\right) \\
\& \operatorname{supp}\left(\pi_{1}\right) \subseteq \operatorname{supp}\left(\pi_{2}\right)
\end{array}\right]
$$

In other words, every block of $\pi_{1}$ is covered by a union of blocks of $\pi_{2}$, and every block of $\pi_{2}$ either contains a block of $\pi_{1}$ or has at least one point not belonging to any block of $\pi_{1}$.

Lemma 11 Let $\pi_{1}, \pi_{2} \in \Pi^{*}(E)$. Then

$$
\pi_{1} \stackrel{\mathrm{a}}{\leq} \pi_{2} \Longleftrightarrow\left[\pi_{1} \stackrel{\mathrm{e}}{\leq} \pi_{2} \& \operatorname{supp}\left(\pi_{1}\right)=\operatorname{supp}\left(\pi_{2}\right)\right]
$$

and

$$
\left[\pi_{0} \stackrel{\mathrm{e}}{\leq} \pi_{1} \stackrel{\mathrm{e}}{\leq} \pi_{2} \quad \& \pi_{0} \stackrel{\mathrm{a}}{\leq} \pi_{2}\right] \Longrightarrow \pi_{0} \stackrel{\mathrm{a}}{\leq} \pi_{1} \stackrel{\mathrm{a}}{\leq} \pi_{2}
$$


Proof If $\operatorname{supp}\left(\pi_{1}\right)=\operatorname{supp}\left(\pi_{2}\right)$, then $\pi_{2} \cap \mathcal{P}\left(\operatorname{supp}\left(\pi_{1}\right)\right)=\pi_{2} \cap \mathcal{P}\left(\operatorname{supp}\left(\pi_{2}\right)\right)=\pi_{2}$. Let $\pi_{1} \stackrel{\text { a }}{\leq} \pi_{2}$; then $\operatorname{supp}\left(\pi_{1}\right)=\operatorname{supp}\left(\pi_{2}\right)$, so $\pi_{1} \Subset \pi_{2}=\pi_{2} \cap \mathcal{P}\left(\operatorname{supp}\left(\pi_{1}\right)\right)$; hence $\pi_{1} \stackrel{\mathrm{e}}{\leq} \pi_{2}$. Conversely, let $\pi_{1} \stackrel{\mathrm{e}}{\leq} \pi_{2}$ and $\operatorname{supp}\left(\pi_{1}\right)=\operatorname{supp}\left(\pi_{2}\right)$; then $\pi_{1} \Subset \pi_{2} \cap \mathcal{P}\left(\operatorname{supp}\left(\pi_{1}\right)\right)=\pi_{2}$; hence $\pi_{1} \stackrel{\text { a }}{\leq} \pi_{2}$. Therefore (22) holds.

Now let $\pi_{0} \stackrel{\text { e }}{\leq} \pi_{1} \stackrel{\text { e }}{\leq} \pi_{2}$ and $\pi_{0} \stackrel{\text { a }}{\leq} \pi_{2}$. Then $\operatorname{supp}\left(\pi_{0}\right) \subseteq \operatorname{supp}\left(\pi_{1}\right) \subseteq \operatorname{supp}\left(\pi_{2}\right)$ and $\operatorname{supp}\left(\pi_{0}\right)=\operatorname{supp}\left(\pi_{2}\right)$, so $\operatorname{supp}\left(\pi_{0}\right)=\operatorname{supp}\left(\pi_{1}\right)=\operatorname{supp}\left(\pi_{2}\right)$, hence (22) gives $\pi_{0} \stackrel{\mathrm{a}}{\leq} \pi_{1} \stackrel{\mathrm{a}}{\leq} \pi_{2}$. Therefore (23) holds.

We will now give the main properties of the extended order:

Theorem 12 The extended order is a partial order relation on $\Pi^{*}(E)$; it contains the apportioning-inflating and standard orders: for any $\pi_{1}, \pi_{2} \in \Pi^{*}(E), \pi_{1} \stackrel{\text { ai }}{\leq} \pi_{2} \Rightarrow \pi_{1} \stackrel{\mathrm{e}}{\leq}$ $\pi_{2}$ and $\pi_{1} \leq \pi_{2} \Rightarrow \pi_{1} \stackrel{e}{\leq} \pi_{2}$. It is generated by inclusion followed by apportioning:

$$
\pi_{1} \stackrel{\mathrm{e}}{\leq} \pi_{2} \Longleftrightarrow \exists \pi \in \Pi^{*}(E), \pi_{1} \subseteq \pi \stackrel{\mathrm{a}}{\leq} \pi_{2}
$$

The greatest and least elements are $\mathbf{1}_{E}$ and $\varnothing$. The covering relation is $\stackrel{\mathrm{a}}{\prec} \cup \stackrel{\mathrm{s}}{\prec}$.

Let $E$ be finite. Then $\left(\Pi^{*}(E), \stackrel{\mathrm{e}}{\leq}\right)$ is graded by $\left(h_{m}, h_{s}\right)$ for $(\stackrel{\mathrm{a}}{\prec}, \stackrel{\mathrm{s}}{\prec})$, that is, for any $\pi_{1}, \pi_{2} \in \Pi^{*}(E)$ we have:

$$
\begin{aligned}
& \pi_{1} \stackrel{\mathrm{e}}{<} \pi_{2} \Longrightarrow\left[\begin{array}{l}
h_{m}\left(\pi_{1}\right) \leq h_{m}\left(\pi_{2}\right) \& \\
h_{s}\left(\pi_{1}\right) h_{s}\left(\pi_{2}\right) \& \\
h\left(\pi_{1}\right)<h\left(\pi_{2}\right)
\end{array}\right], \\
& \pi_{1} \stackrel{\mathrm{a}}{\prec} \pi_{2} \Longrightarrow\left[\begin{array}{l}
h_{s}\left(\pi_{2}\right)=h_{s}\left(\pi_{1}\right) \& \\
h_{m}\left(\pi_{2}\right)=h_{m}\left(\pi_{1}\right)+1
\end{array}\right], \\
& \pi_{1} \stackrel{\mathrm{s}}{\prec} \pi_{2} \Longrightarrow\left[\begin{array}{l}
h_{m}\left(\pi_{2}\right)=h_{m}\left(\pi_{1}\right) \& \\
h_{s}\left(\pi_{2}\right)=h_{s}\left(\pi_{1}\right)+1
\end{array}\right] .
\end{aligned}
$$

In particular, $\left(\Pi^{*}(E), \stackrel{\mathrm{e}}{\leq}\right)$ is graded by $h=h_{m}+h_{s}$. Also $h_{m}(\varnothing)=h_{s}(\varnothing)=0$, and for $\pi \in \Pi^{*}(E)$, the height of $\pi$ is $h(\pi)$.

Proof Let $\pi_{0}, \pi_{1}, \pi_{2} \in \Pi^{*}(E)$. As $\pi_{0} \stackrel{\text { a }}{\leq} \pi_{0},(22)$ gives $\pi_{0} \stackrel{\text { e }}{\leq} \pi_{0}$ : hence $\stackrel{\text { e }}{\leq}$ is reflexive. If $\pi_{0} \stackrel{e}{\leq} \pi_{1}$ and $\pi_{1} \stackrel{e}{\leq} \pi_{0}$, then by double inclusion $\operatorname{supp}\left(\pi_{0}\right)=\operatorname{supp}\left(\pi_{1}\right)$, and $(22)$ gives $\pi_{0} \stackrel{\text { a }}{\leq} \pi_{1}$ and $\pi_{1} \stackrel{\text { a }}{\leq} \pi_{0}$, so the antisymmetry of $\stackrel{\text { a }}{\leq}$ gives $\pi_{0}=\pi_{1}$ : hence $\stackrel{\text { e }}{\leq}$ is antisymmetric. If $\pi_{0} \stackrel{\mathrm{e}}{\leq} \pi_{1} \stackrel{\mathrm{e}}{\leq} \pi_{2}$, we have $\operatorname{supp}\left(\pi_{0}\right) \subseteq \operatorname{supp}\left(\pi_{1}\right) \subseteq \operatorname{supp}\left(\pi_{2}\right), \pi_{0} \Subset \pi_{1} \cap$ $\mathcal{P}\left(\operatorname{supp}\left(\pi_{0}\right)\right)$ and $\pi_{1} \Subset \pi_{2} \cap \mathcal{P}\left(\operatorname{supp}\left(\pi_{1}\right)\right)$; by $(11)$, the latter gives $\pi_{1} \cap \mathcal{P}\left(\operatorname{supp}\left(\pi_{0}\right)\right) \Subset$ $\pi_{2} \cap \mathcal{P}\left(\operatorname{supp}\left(\pi_{1}\right)\right) \cap \mathcal{P}\left(\operatorname{supp}\left(\pi_{0}\right)\right)=\pi_{2} \cap \mathcal{P}\left(\operatorname{supp}\left(\pi_{0}\right)\right) ;$ thus $\pi_{0} \Subset \pi_{1} \cap \mathcal{P}\left(\operatorname{supp}\left(\pi_{0}\right)\right) \Subset$ $\pi_{2} \cap \mathcal{P}\left(\operatorname{supp}\left(\pi_{0}\right)\right)$, and the transitivity of $\Subset$ gives then $\pi_{0} \Subset \pi_{2} \cap \mathcal{P}\left(\operatorname{supp}\left(\pi_{0}\right)\right)$; as $\operatorname{supp}\left(\pi_{0}\right) \subseteq \operatorname{supp}\left(\pi_{2}\right)$, we get $\pi_{0} \stackrel{\mathrm{e}}{\leq} \pi_{2}$ : hence $\stackrel{\mathrm{e}}{\leq}$ is transitive. Therefore $\stackrel{\mathrm{e}}{\leq}$ is partial order relation on $\Pi^{*}(E)$.

If $\pi_{1} \stackrel{\text { ai }}{\leq} \pi_{2}$, then $\operatorname{supp}\left(\pi_{1}\right) \subseteq \operatorname{supp}\left(\pi_{2}\right)$ and $\pi_{1} \Subset \pi_{2} ;$ now $\pi_{2} \supseteq \pi_{2} \cap \mathcal{P}\left(\operatorname{supp}\left(\pi_{1}\right)\right)$, so $\pi_{2} \Subset \pi_{2} \cap \mathcal{P}\left(\operatorname{supp}\left(\pi_{1}\right)\right)$ (since $\Subset$ contains $\supseteq$, see Proposition 2 ), and by transitivity of $\Subset$ we get $\pi_{1} \Subset \pi_{2} \cap \mathcal{P}\left(\operatorname{supp}\left(\pi_{1}\right)\right)$; thus $\pi_{1} \stackrel{\mathrm{e}}{\leq} \pi_{2}$. Hence $\stackrel{\mathrm{e}}{\leq}$ contains $\stackrel{\text { ai }}{\leq}$. If $\pi_{1} \leq \pi_{2}$, then $\operatorname{supp}\left(\pi_{1}\right) \subseteq \operatorname{supp}\left(\pi_{2}\right)$ and $(10)$ gives $\pi_{1} \Subset \pi_{2} \cap \mathcal{P}\left(\operatorname{supp}\left(\pi_{1}\right)\right)$; hence $\pi_{1} \stackrel{\mathrm{e}}{\leq} \pi_{2}$. Therefore $\stackrel{\mathrm{e}}{\leq}$ contains $\leq$. 


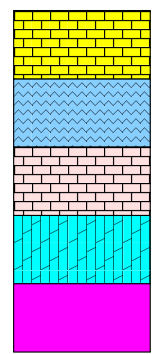

$\pi_{1}$

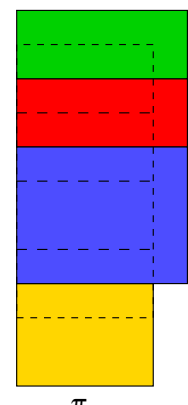

$\pi_{2}$

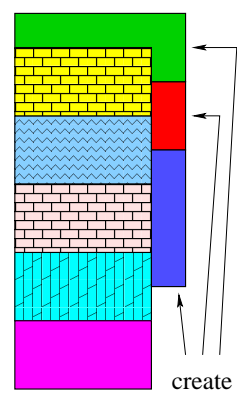

$\pi$

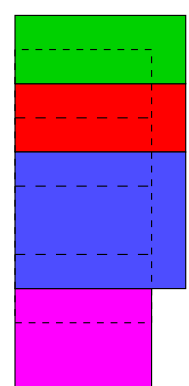

apportion

Fig. 13 From left to right: first $\pi_{1}$, second $\pi_{2}$, where $\pi_{1} \stackrel{e}{\leq} \pi_{2}$; the blocks of $\pi_{1}$ that are not included in a block of $\pi_{2}$ will be apportioned, they are shown hatched. Third, $\pi$ is obtained by adding to $\pi_{1}$ the blocks $B \backslash \operatorname{supp}\left(\pi_{1}\right)$ for all $B \in \pi_{2} \backslash \mathcal{P}\left(\operatorname{supp}\left(\pi_{1}\right)\right)$, thus $\pi_{1} \subseteq \pi$; fourth, $\pi \stackrel{\text { a }}{\leq} \pi_{2}$.

If $\pi_{1} \subseteq \pi \stackrel{\text { a }}{\leq} \pi_{2}$, then $\pi_{1} \leq \pi \stackrel{\text { ai }}{\leq} \pi_{2}$, so $\pi_{1} \stackrel{\mathrm{e}}{\leq} \pi \stackrel{\mathrm{e}}{\leq} \pi_{2}$; by transitivity we get $\pi_{1} \stackrel{\mathrm{e}}{\leq} \pi_{2}$. Conversely, suppose that $\pi_{1} \stackrel{\mathrm{e}}{\leq} \pi_{2}$; let $\pi^{\prime}=\left\{B \backslash \operatorname{supp}\left(\pi_{1}\right) \mid B \in \pi_{2} \backslash \mathcal{P}\left(\operatorname{supp}\left(\pi_{1}\right)\right)\right\}$; then $\pi^{\prime} \in \Pi^{*}(E)$ and $\operatorname{supp}\left(\pi^{\prime}\right) \cap \operatorname{supp}\left(\pi_{1}\right)=\emptyset$; thus $\pi=\pi_{1} \cup \pi^{\prime} \in \Pi^{*}(E)$. Then $\pi_{1} \subseteq \pi$ by construction. As $\pi_{1} \Subset \pi_{2} \cap \mathcal{P}\left(\operatorname{supp}\left(\pi_{1}\right)\right)$, every block of $\pi_{2} \cap \mathcal{P}\left(\operatorname{supp}\left(\pi_{1}\right)\right)$ contains a block of $\pi_{1}$, while for $B \in \pi_{2} \backslash \mathcal{P}\left(\operatorname{supp}\left(\pi_{1}\right)\right), B$ contains $B \backslash \operatorname{supp}\left(\pi_{1}\right) \in \pi^{\prime}$; thus every block of $\pi_{2}$ contains a block of $\pi_{1} \cup \pi^{\prime}$, that is, $\pi \Subset \pi_{2}$. Now for $B \in \pi_{2} \cap \mathcal{P}\left(\operatorname{supp}\left(\pi_{1}\right)\right)$, $B \backslash \operatorname{supp}\left(\pi_{1}\right)=\emptyset$, while for $B \in \pi_{2} \backslash \mathcal{P}\left(\operatorname{supp}\left(\pi_{1}\right)\right), B \backslash \operatorname{supp}\left(\pi_{1}\right) \in \pi^{\prime}$, so

$$
\begin{gathered}
\operatorname{supp}\left(\pi_{2}\right) \backslash \operatorname{supp}\left(\pi_{1}\right)=\bigcup_{B \in \pi_{2}}\left(B \backslash \operatorname{supp}\left(\pi_{1}\right)\right) \\
=\bigcup\left\{B \backslash \operatorname{supp}\left(\pi_{1}\right) \mid B \in \pi_{2} \backslash \mathcal{P}\left(\operatorname{supp}\left(\pi_{1}\right)\right)\right\}=\operatorname{supp}\left(\pi^{\prime}\right),
\end{gathered}
$$

and $\operatorname{as} \operatorname{supp}\left(\pi_{1}\right) \subseteq \operatorname{supp}\left(\pi_{2}\right)$, we get

$$
\operatorname{supp}(\pi)=\operatorname{supp}\left(\pi_{1}\right) \cup \operatorname{supp}\left(\pi^{\prime}\right)=\operatorname{supp}\left(\pi_{1}\right) \cup\left(\operatorname{supp}\left(\pi_{2}\right) \backslash \operatorname{supp}\left(\pi_{1}\right)\right)=\operatorname{supp}\left(\pi_{2}\right) .
$$

Since $\pi \Subset \pi_{2}$ and $\operatorname{supp}(\pi)=\operatorname{supp}\left(\pi_{2}\right)$, we deduce that $\pi \stackrel{\text { a }}{\leq} \pi_{2}$. See Figure 13 .

For any $\pi \in \Pi^{*}(E), \pi \cap \mathcal{P}(\operatorname{supp}(\varnothing))=\pi \cap \mathcal{P}(\emptyset)=\varnothing$, with $\varnothing \Subset \varnothing$, thus $\varnothing \Subset \pi \cap \mathcal{P}(\emptyset)$; now $\operatorname{supp}(\varnothing)=\emptyset \subseteq \operatorname{supp}(\pi)$, hence $\varnothing \stackrel{\mathrm{e}}{\leq} \pi$. Thus $\varnothing$ is the least element for the order $\stackrel{\mathrm{e}}{\leq}$. Next, we have two cases:

$-\operatorname{supp}(\pi)=E$, so $\pi \neq \varnothing$, hence by Proposition $3, \pi \Subset \mathbf{1}_{E}$, with $\mathbf{1}_{E} \cap \mathcal{P}(\operatorname{supp}(\pi))=$ $\mathbf{1}_{E}$;

$-\operatorname{supp}(\pi) \subset E$ and by Proposition $3, \pi \Subset \varnothing$, with $\mathbf{1}_{E} \cap \mathcal{P}(\operatorname{supp}(\pi))=\varnothing$;

hence in both cases $\pi \Subset \mathbf{1}_{E} \cap \mathcal{P}(\operatorname{supp}(\pi))$; since $\operatorname{supp}(\pi) \subseteq E=\operatorname{supp}\left(\mathbf{1}_{E}\right)$, we get $\pi \stackrel{\mathrm{e}}{\leq} \mathbf{1}_{E}$. Thus $\mathbf{1}_{E}$ is the greatest element for the order $\stackrel{\mathrm{e}}{\leq}$.

Let $\pi_{1} \stackrel{\text { a }}{\prec} \pi_{2}$. If $\pi_{1} \stackrel{\mathrm{e}}{\leq} \pi \stackrel{\mathrm{e}}{\leq} \pi_{2}$, then (23) gives $\pi_{1} \stackrel{\mathrm{a}}{\leq} \pi \stackrel{\mathrm{a}}{\leq} \pi_{2}$. But $\stackrel{\mathrm{a}}{\prec}$ is the covering relation for $\stackrel{\text { a }}{\leq}$, so $\pi=\pi_{1}$ or $\pi=\pi_{2}$. Therefore $\pi_{2}$ covers $\pi_{1}$ for the order $\stackrel{\mathrm{e}}{\leq}$.

Let now $\pi_{1} \stackrel{\mathrm{s}}{\prec} \pi_{2}$; by (5) there is $p \in E \backslash \operatorname{supp}\left(\pi_{1}\right)$ such that $\pi_{2}=\pi_{1} \cup\{\{p\}\}$; then $\pi_{2} \cap \mathcal{P}\left(\operatorname{supp}\left(\pi_{1}\right)\right)=\pi_{1}$. Suppose that $\pi_{1} \stackrel{\mathrm{e}}{\leq} \pi \stackrel{\mathrm{e}}{\leq} \pi_{2}$. From the above proof 
of the transitivity of $\stackrel{\mathrm{e}}{\leq}$ we have $\pi_{1} \Subset \pi \cap \mathcal{P}\left(\operatorname{supp}\left(\pi_{1}\right)\right) \Subset \pi_{2} \cap \mathcal{P}\left(\operatorname{supp}\left(\pi_{1}\right)\right)$, thus $\pi_{1} \Subset \pi \cap \mathcal{P}\left(\operatorname{supp}\left(\pi_{1}\right)\right) \Subset \pi_{1}$, and the antisymmetry of $\Subset$ gives $\pi \cap \mathcal{P}\left(\operatorname{supp}\left(\pi_{1}\right)\right)=\pi_{1}$. If $\operatorname{supp}(\pi)=\operatorname{supp}\left(\pi_{1}\right)$, then $\pi=\pi_{1}$. If $\operatorname{supp}(\pi) \neq \operatorname{supp}\left(\pi_{1}\right)$, as $\operatorname{supp}\left(\pi_{1}\right) \subseteq \operatorname{supp}(\pi) \subseteq$ $\operatorname{supp}\left(\pi_{2}\right)=\operatorname{supp}\left(\pi_{1}\right) \cup\{p\}$, we obtain that $\operatorname{supp}(\pi)=\operatorname{supp}\left(\pi_{1}\right) \cup\{p\}=\operatorname{supp}\left(\pi_{2}\right)$; now $\pi \Subset \pi_{2} \cap \mathcal{P}(\operatorname{supp}(\pi))=\pi_{2}$, so the block $\{p\} \in \pi_{2}$ contains a block of $\pi$, which must thus be itself; since $\pi_{1}=\pi \cap \mathcal{P}\left(\operatorname{supp}\left(\pi_{1}\right)\right) \subseteq \pi$ and $\{p\} \in \pi$, we get $\pi_{2}=\pi_{1} \cup\{\{p\}\} \subseteq \pi$, ans as $\operatorname{supp}(\pi)=\operatorname{supp}\left(\pi_{2}\right)$, we deduce that $\pi=\pi_{2}$. We have thus shown that if $\pi_{1} \stackrel{\mathrm{s}}{\prec} \pi_{2}$ and $\pi_{1} \stackrel{\mathrm{e}}{\leq} \pi \stackrel{\mathrm{e}}{\leq} \pi_{2}$, then $\pi=\pi_{1}$ or $\pi=\pi_{2}$, therefore $\pi_{2}$ covers $\pi_{1}$ for the order $\stackrel{\mathrm{e}}{\leq}$.

Conversely, let $\pi_{2}$ cover $\pi_{1}$ for $\stackrel{\mathrm{e}}{\leq}$. By the above, we have $\pi_{1} \subseteq \pi \stackrel{\text { a }}{\leq} \pi_{2}$. The three cases $\pi_{1} \subset \pi \stackrel{\mathrm{a}}{<} \pi_{2}, \pi_{1}=\pi \stackrel{\mathrm{a}}{<} \pi^{\prime} \stackrel{\mathrm{a}}{<} \pi_{2}$ and $\pi_{1} \subset \pi^{\prime} \subset \pi=\pi_{2}$ contradict the covering of $\pi_{1}$ by $\pi_{2}$, so there remain only the cases where $\pi_{2}$ covers $\pi_{1}$ for $\stackrel{\text { a }}{\leq}$ or for $\subseteq$, that is, $\pi_{1} \stackrel{\text { a }}{\prec} \pi_{2}$ or $\pi_{1} \stackrel{c}{\prec} \pi_{2}$ (see Theorem 9 and Table 1 ). Now if $\pi_{1} \stackrel{c}{\prec} \pi_{2}$, then $\pi_{1}<\pi_{2}$, so $\pi_{2}$ must cover $\pi_{1}$ for the order $\leq$, and by Table 2 we get $\pi_{1} \stackrel{\text { s }}{\prec} \pi_{2}$, because $\stackrel{\mathrm{m}}{\prec}$ requires support equality, which is incompatible with $\stackrel{\mathrm{c}}{\prec}$. Therefore the covering relation is $\stackrel{\mathrm{a}}{\prec} \cup \stackrel{\mathrm{s}}{\prec}$.

Let $E$ be finite. For $\pi_{1} \stackrel{\mathrm{e}}{\leq} \pi_{2}$, we have $\pi_{1} \subseteq \pi \stackrel{\text { a }}{\leq} \pi_{2}$. Since $\pi_{1} \leq \pi$, by Table 2 we have $h_{m}\left(\pi_{1}\right) \leq h_{m}(\pi)$ and $h_{s}\left(\pi_{1}\right) \leq h_{s}(\pi)$; by Theorem $9, h_{m}(\pi) \leq h_{m}\left(\pi_{2}\right)$ and $h_{s}(\pi)=h_{s}\left(\pi_{2}\right)$. Thus $h_{m}\left(\pi_{2}\right) \geq h_{m}\left(\pi_{1}\right)$ and $h_{s}\left(\pi_{2}\right) \geq h_{s}\left(\pi_{1}\right)$, so (9) gives

$$
h\left(\pi_{2}\right)-h\left(\pi_{1}\right)=\left(h_{m}\left(\pi_{2}\right)-h_{m}\left(\pi_{1}\right)\right)+\left(h_{s}\left(\pi_{2}\right)-h_{s}\left(\pi_{1}\right)\right) \geq 0,
$$

with both terms $h_{m}\left(\pi_{2}\right)-h_{m}\left(\pi_{1}\right)$ and $h_{s}\left(\pi_{2}\right)-h_{s}\left(\pi_{1}\right)$ being $\geq 0$. If $h\left(\pi_{2}\right)=$ $h\left(\pi_{1}\right)$, then $h_{m}\left(\pi_{2}\right)=h_{m}\left(\pi_{1}\right)$ and $h_{s}\left(\pi_{2}\right)=h_{s}\left(\pi_{1}\right) ;$ as $\operatorname{supp}\left(\pi_{1}\right) \subseteq \operatorname{supp}\left(\pi_{2}\right)$ and $\left|\operatorname{supp}\left(\pi_{1}\right)\right|=h_{s}\left(\pi_{1}\right)=h_{s}\left(\pi_{2}\right)=\left|\operatorname{supp}\left(\pi_{2}\right)\right|$, it follows that $\operatorname{supp}\left(\pi_{1}\right)=\operatorname{supp}\left(\pi_{2}\right)$, hence $\pi_{1} \stackrel{\text { a }}{\leq} \pi_{2}$ by $(22)$; now since $h_{m}\left(\pi_{2}\right)=h_{m}\left(\pi_{1}\right)$, Theorem 9 gives $\pi_{1}=\pi_{2}$. Hence $\pi_{1} \stackrel{\mathrm{e}}{<} \pi_{2} \Rightarrow h\left(\pi_{1}\right)<h\left(\pi_{2}\right)$, with $h_{s}\left(\pi_{1}\right) \leq h_{s}\left(\pi_{2}\right)$ and $h_{c}\left(\pi_{1}\right) \geq h_{c}\left(\pi_{2}\right)$. Now if $\pi_{1} \stackrel{\gtrless}{\prec} \pi_{2}$, then $h_{s}\left(\pi_{2}\right)=h_{s}\left(\pi_{1}\right)$ and $h_{m}\left(\pi_{2}\right)=h_{m}\left(\pi_{1}\right)+1$ by Theorem 9 , while if $\pi_{1} \stackrel{\mathrm{s}}{\prec} \pi_{2}$, then $h_{m}\left(\pi_{2}\right)=h_{m}\left(\pi_{1}\right)$ and $h_{s}\left(\pi_{2}\right)=h_{s}\left(\pi_{1}\right)+1$ by Table 2 . Therefore $\left(\Pi^{*}(E), \stackrel{\mathrm{e}}{\leq}\right)$ is graded by $\left(h_{m}, h_{s}\right)$ for $(\stackrel{\mathrm{a}}{\prec}, \stackrel{\mathrm{s}}{\prec})$; by Proposition 1 , it is graded by $h=h_{m}+h_{s}$.

Since $h_{m}(\varnothing)=h_{s}(\varnothing)=0$ by Table $2, h(\varnothing)=0$, hence for $\pi \in \Pi^{*}(E)$, the height of $\pi$ is $h(\pi)-h(\varnothing)=h(\pi)$.

It follows that the extended order contains all the orders on $\Pi^{*}(E)$ that we have considered, except the building order, namely: the standard order, the merging, inclusion, inflating, merging-inflating and inclusion-inflating orders introduced in [16] (cf. Subsection 2.2), and the apportioning and apportioning-inflating orders introduced in Subsection 3.1. However the extended and building orders are incomparable, since $\stackrel{e}{\leq}$ contains the inclusion $\subseteq$, while $\Subset$ contains the inverse inclusion $\supseteq$.

We saw above the similarity of Theorems 9 and 10 with the corresponding results for the merging and merging-inflating orders. Again, Theorem 12 is similar to the corresponding statement for the standard order (cf. Table 2, see also The- 
orems 2 and 7 of [16]), where we only have to replace $\stackrel{\mathrm{m}}{\leq}, \stackrel{\mathrm{mi}}{\leq}, \leq$ and $\stackrel{\mathrm{m}}{\prec}$ by $\stackrel{\text { a }}{\leq}, \stackrel{\text { ai }}{\leq}, \stackrel{\mathrm{e}}{\leq}$ and $\stackrel{\text { a }}{\prec}$. Compare also the present Figure 13 with Figure 6 of [16].

We will now pursue further this analogy between the merging and standard orders on the one hand, the apportioning and extended orders on the other hand. Here we will obtain the counterparts of some identities and relations between orders. Recall the definition (1) of the merging order:

$$
\forall \pi_{1}, \pi_{2} \in \Pi^{*}(E), \quad \pi_{1} \leq \pi_{2} \Longleftrightarrow\left[\pi_{1} \leq \pi_{2} \& \operatorname{supp}\left(\pi_{1}\right)=\operatorname{supp}\left(\pi_{2}\right)\right]
$$

Its counterpart is (22). In equation (18) of [16], we showed that

$$
\forall \pi_{0}, \pi_{1}, \pi_{2} \in \Pi^{*}(E), \quad\left[\pi_{0} \leq \pi_{1} \leq \pi_{2} \& \pi_{0} \stackrel{\mathrm{m}}{\leq} \pi_{2}\right] \Longrightarrow \pi_{0} \stackrel{\mathrm{m}}{\leq} \pi_{1} \stackrel{\mathrm{m}}{\leq} \pi_{2}
$$

Its counterpart is (23). Next, recall the definition (3) of the merging-inflating order:

$$
\forall \pi_{1}, \pi_{2} \in \Pi^{*}(E), \quad \pi_{1} \leq \pi_{2} \Longleftrightarrow\left[\pi_{1} \leq \pi_{2} \& \pi_{1} \Subset \pi_{2}\right] .
$$

It has a counterpart with the apportioning-inflating and extended orders in place of the merging-inflating and standard orders:

$$
\forall \pi_{1}, \pi_{2} \in \Pi^{*}(E), \quad \pi_{1} \stackrel{\text { ai }}{\leq} \pi_{2} \Longleftrightarrow\left[\pi_{1} \stackrel{\mathrm{e}}{\leq} \pi_{2} \& \pi_{1} \Subset \pi_{2}\right]
$$

This follows immediately from the definitions (20) and (21) of the apportioninginflating and extended orders and the fact that $\pi_{1} \Subset \pi_{2} \Rightarrow \pi_{1} \Subset \pi_{2} \cap \mathcal{P}\left(\operatorname{supp}\left(\pi_{1}\right)\right)$.

However some relations between the merging-inflating and standard orders have no counterparts with the apportioning-inflating and extended orders. For instance, in equations $(25,26)$ of $[16]$, we showed that

$$
\begin{aligned}
& \forall \pi_{0}, \pi_{1}, \pi_{2} \in \Pi^{*}(E), \quad\left[\pi_{0} \leq \pi_{1} \leq \pi_{2} \& \pi_{0} \leq \pi_{2}\right] \Longrightarrow \quad \pi_{1}{ }^{\mathrm{mi}} \pi_{2} \\
& \text { and }\left[\pi_{0} \stackrel{\mathrm{mi}}{\leq} \pi_{1} \leq \pi_{2} \& \pi_{0} \stackrel{\mathrm{i}}{\leq} \pi_{2}\right] \Longrightarrow \pi_{0} \stackrel{\mathrm{i}}{\leq} \pi_{1} \stackrel{\mathrm{i}}{\leq} \pi_{2} \text {. }
\end{aligned}
$$

Now let $\{A, B, C, D, E, F\} \in \Pi^{*}(E)$ (that is, $A, B, C, D, E, F$ are mutually disjoint non-empty subsets of $E)$. Define

$$
\pi_{0}=\{A, B\}, \quad \pi_{1}=\{A \cup B \cup C \cup D\}, \quad \pi_{2}=\{A \cup C \cup E, B \cup D \cup F\}
$$

Then $\pi_{0} \stackrel{\mathrm{mi}}{\leq} \pi_{1} \stackrel{\mathrm{e}}{\leq} \pi_{2}, \pi_{0} \stackrel{\mathrm{i}}{\leq} \pi_{2}, \pi_{0} \stackrel{\mathrm{i}}{\not} \pi_{1}$ and $\pi_{1} \stackrel{\text { ai }}{\dot{\mathrm{x}}} \pi_{2}$.

We end this section by extending Theorem 15 and Figure 9 of [16]:

Theorem 13 The lattice of partial order relations on $\Pi^{*}(E)$ generated by the apportioning, merging, inclusion and inflating orders contains these four orders, the merginginflating, inclusion-inflating, apportioning-inflating, standard and extended orders, and the identity. Its Hasse diagram is that of Figure 14. 


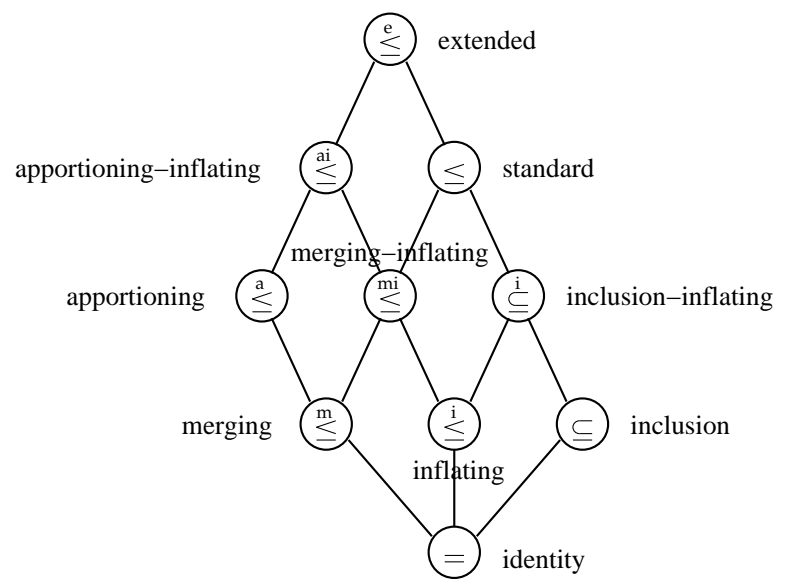

Fig. 14 Hasse diagram of the lattice of partial orders on $\Pi^{*}(E)$ generated by the apportioning, merging, inclusion and inflating orders.

Proof Let $\mathcal{O}=\{=, \stackrel{\mathrm{m}}{\leq}, \stackrel{\mathrm{i}}{\leq}, \subseteq, \stackrel{\mathrm{mi}}{\leq}, \stackrel{\mathrm{i}}{\leq}, \leq\}$ and $\mathcal{O}^{\prime}=\mathcal{O} \cup\{\stackrel{\mathrm{a}}{\leq}, \stackrel{\text { ai }}{\leq}, \stackrel{\mathrm{e}}{\leq}\}$. As in the proof of Theorem 15 of [16], in order to avoid confusion with the elements $\subseteq$ and $=$ of $\mathcal{O}$ we will use the symbols $\subseteq$ and $\equiv$ for the inclusion and equality between binary relations on $\Pi^{*}(E)$. Now $\mathcal{O}^{\prime}$ is a part of the lattice $O(\stackrel{e}{\leq})$ of all partial order relations on included in $\stackrel{\mathrm{e}}{\leq}$; the non-empty infimum in $O(\stackrel{\mathrm{e}}{\leq})$ is the intersection; we will write $\sqcup$ for the binary supremum operation in $O(\stackrel{\mathrm{e}}{\leq})$.

By Theorem 15 of [16], $\mathcal{O}$ is a lattice, it is generated by the merging, inclusion and inflating orders, and its Hasse diagram (cf. Figure 9 of [16]) is the restriction of Figure 14 to $\mathcal{O}$. Thus we need only to prove the statement for the relations involving the orders $\stackrel{\text { a }}{\leq}, \stackrel{\text { ai }}{\leq}$ and $\stackrel{\text { e }}{\leq}$.

We know from Theorems 10 and 12 that $\stackrel{\text { a }}{\leq} \sqcup \stackrel{\text { i }}{\leq} \equiv \stackrel{\text { ai }}{\leq}$ and $\stackrel{\text { a }}{\leq} \sqcup \subseteq \equiv \stackrel{\text { e }}{\leq}$. Since $\mathcal{O}$ is generated by $\stackrel{m}{\leq}, \stackrel{i}{\leq}$ and $\subseteq, \mathcal{O}^{\prime}$ will be generated by these three and $\stackrel{\text { a }}{\leq}$.

The edges in Figure 14 involving the nodes $\stackrel{\text { a }}{\leq}, \stackrel{\text { ai }}{\leq}$ and $\stackrel{\text { e }}{\leq}$ correspond indeed to the inclusion relations $\stackrel{\mathrm{m}}{\leq} \subseteq \stackrel{\text { a }}{\leq}, \stackrel{\text { a }}{\leq} \subseteq \stackrel{\text { ai }}{\leq}, \stackrel{\text { mi }}{\leq} \subseteq \stackrel{\text { ai }}{\leq}, \stackrel{\text { ai }}{\leq} \subseteq \stackrel{\text { e }}{\leq}$ and $\leq \subseteq \stackrel{\text { e }}{\leq}$.

Let $R_{1} \equiv \stackrel{\text { a }}{\leq}$ or $\stackrel{\text { ai }}{\leq}$ and let $R_{2} \in \mathcal{O}$ that is incomparable with $R_{1}$. If $R_{2} \supseteqq \subseteq$, then $R_{1} \sqcup R_{2} \supseteqq \stackrel{\mathrm{a}}{\leq} \sqcup \subseteq \equiv \stackrel{\mathrm{e}}{\leq}$, thus $R_{1} \sqcup R_{2} \equiv \stackrel{\mathrm{e}}{\leq}$, as in Figure 14. If $R_{2} \nsupseteq \subseteq$, then $R_{2} \subseteq \stackrel{\text { mi }}{\leq}$, and the incomparability of $R_{1}$ and $R_{2}$ gives $R_{1} \equiv \stackrel{\text { a }}{\leq}$ and $R_{2} \equiv \stackrel{\text { i }}{\leq}$ or $\stackrel{\text { mi }}{\leq}$; now $\stackrel{\text { ai }}{\leq} \equiv \stackrel{\text { a }}{\leq} \sqcup \stackrel{\text { i }}{\leq} \equiv \stackrel{\text { a }}{\leq} \sqcup \stackrel{\text { mi }}{\leq}$, as in Figure 14. Hence the supremum operation $\sqcup$ follows Figure 14 .

Let us now consider $R_{1} \cap R_{2}$. First note that $\stackrel{\mathrm{a}}{\leq} \cap \leq \equiv \stackrel{\mathrm{m}}{\leq}$; indeed $\pi_{1} \stackrel{\mathrm{m}}{\leq} \pi_{2}$ means that $\pi_{1} \leq \pi_{2}$ and $\operatorname{supp}\left(\pi_{1}\right)=\operatorname{supp}\left(\pi_{2}\right)$, and it implies that $\pi_{1} \Subset \pi_{2}$; now $\pi_{1} \Subset \pi_{2}$ and $\operatorname{supp}\left(\pi_{1}\right)=\operatorname{supp}\left(\pi_{2}\right)$ together mean that $\pi_{1} \stackrel{\text { a }}{\leq} \pi_{2}$. Next note that $\stackrel{\text { ai }}{\leq} \cap \leq \equiv \stackrel{\text { mi }}{\leq}$; indeed $\pi_{1} \stackrel{\text { mi }}{\leq} \pi_{2}$ means that $\pi_{1} \Subset \pi_{2}$ and $\pi_{1} \leq \pi_{2}$, and the latter implies $\operatorname{supp}\left(\pi_{1}\right) \subseteq \operatorname{supp}\left(\pi_{2}\right) ;$ now $\pi_{1} \Subset \pi_{2}$ and $\operatorname{supp}\left(\pi_{1}\right) \subseteq \operatorname{supp}\left(\pi_{2}\right)$ together mean 
that $\pi_{1} \stackrel{\text { ai }}{\leq} \pi_{2}$. It follows then that $\stackrel{\text { a }}{\leq} \cap R_{2} \equiv \stackrel{\text { a }}{\leq} \cap \leq \cap R_{2} \equiv \stackrel{m}{\leq} \cap R_{2}$, and $\stackrel{\text { ai }}{\leq} \cap R_{2} \equiv \stackrel{\text { ai }}{\leq} \cap \leq \cap R_{2} \equiv \stackrel{\text { mi }}{\leq} \cap R_{2}$; these two relations accord with Figure 14 .

Therefore $\mathcal{O}^{\prime}$ is closed under the binary supremum and infimum (intersection) operations, it is thus a lattice, and these operations on $\mathcal{O}^{\prime}$ follow the diagram of Figure 14.

\section{Discussion, conclusion and perspectives}

We have studied some new orders on partial partitions that are based on Serra's building order, which was introduced in order to describe the elimination of small parasitic classes in an image segmentation (or to filter small noisy zones in an image). We have argued that the building order is too general; in order to be meaningful, it must be restricted to the case where the support of the partial partition is preserved or grows. This has led to a new operation on the blocks of a partial partition, block apportioning; it generalizes block merging. Block apportioning is indeed the global result of Serra's method. We described it in terms of maps defined on the blocks (Lemma 4); we associate to it an invariant (Lemma 6), the apportioning index; in a composition of apportionings, the indexes add up (Proposition 7). In the same way as block merging is associated to the refinement order for partitions (and the merging order for partial partitions), block apportioning corresponds to the apportioning order, defined as the intersection of the building order and of the support equality, which contains the merging order (Theorem 9).

Next we introduced the apportioning-inflating order, the intersection of the building order and of the support inclusion; it is generated by composing apportioning and inflating, and it contains the apportioning and merging-inflating orders (Theorem 10). Finally we constructed the extended order, it is generated by successively creating new blocks then apportioning blocks; it contains the standard and apportioning-inflating orders (Theorem 12).

As seen in Figure 14, we have now on $\Pi^{*}(E)$ ten orders: the identity, merging, inflating, inclusion, merging-inflating, inclusion-inflating, standard, apportioning, apportioning-inflating and extended orders. They are all compatible with support inclusion: when a partial partition grows, the support either grows or remains the same. If we restrict ourselves to $\Pi(A)$, the set of partitions of a fixed support, then there remain only three orders: the identity, merging and apportioning orders. More precisely: (a) the inflating, inclusion and inclusion-inflating orders reduce to the identity; (b) the merging-inflating and standard orders reduce to the merging order; (c) the apportioning-inflating and extended orders reduce to the apportioning order.

The merging, apportioning, inflating and inclusion orders are basic, the other ones are compound, since they are obtained by combination of two basic orders. The basic orders have simple covering relations and gradings, while the compound orders have compound covering relations and gradings.

We saw that the apportioning, apportioning-inflating and extended orders have their main properties similar to those of the merging, merging-inflating and standard orders respectively, we just have to replace block merging by block appor-

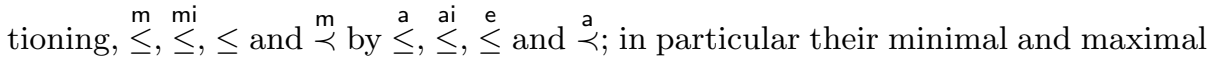
elements and their gradings remain the same. 
The grading ensures that when one constructs iteratively a partial partition from a minimal one by successive elementary growths, the number of elementary operations of each type remains constant. More precisely:

- For the apportioning order $\stackrel{a}{\leq}$ : in every covering chain between $\mathbf{0}_{\operatorname{supp}(\pi)}$ and $\pi$,

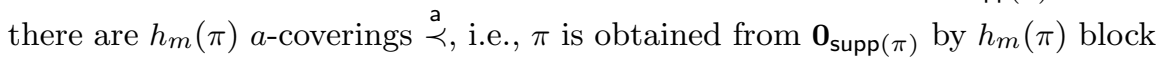
apportionings.

- For the apportioning-inflating order $\leq$ ai given a crossing $A$ of $\pi$, in every covering chain between $\mathbf{0}_{A}$ and $\pi$, there are $|A|-h_{c}(\pi)$ a-coverings $\stackrel{\mathrm{a}}{\prec}$ and $h_{s}(\pi)-|A|$ $i$-coverings $\stackrel{\mathrm{i}}{\prec}$, i.e., $\pi$ is obtained from $\mathbf{0}_{A}$ by $|A|-h_{c}(\pi)$ block apportionings and $h_{s}(\pi)-|A|$ inflations of a block by one point; when $A$ is a transversal of $\pi,|A|=h_{c}(\pi), \mathbf{0}_{A} \stackrel{\mathrm{i}}{\leq} \pi$, there are $h_{m}(\pi) i$-coverings $\stackrel{i}{\prec}$ but no $a$-covering, i.e., $\pi$ is obtained from $\mathbf{0}_{A}$ by $h_{m}(\pi)$ inflations of a block by one point.

- For the extended order $\stackrel{\mathrm{e}}{\leq}$ : in every covering chain between $\emptyset$ and $\pi$, there are $h_{m}(\pi) a$-coverings $\stackrel{\mathrm{a}}{\prec}$ and $h_{s}(\pi) s$-coverings $\stackrel{\mathrm{s}}{\prec}$, i.e., $\pi$ is obtained from $\varnothing$ by $h_{m}(\pi)$ block apportionings and $h_{s}(\pi)$ creations of singleton blocks.

For the six previous orders, a similar description of the growth of a partial partition was given at the end of Section 3 of [16].

A classical problem in the implementation of image processing methods and algorithms is local knowledge: many operations, in particular those that commute with translation, are given for images defined on an unbounded space, but in practice images are defined on a bounded grid, and sometimes the analysis must be done locally within a sliding window called a mask. In the case of segmentation, Serra [21] calls this class permanency: we have to restrict partial partitions to a mask $A \in \mathcal{P}(E)$. This can be done in two ways. We first define the truncation by $A$ :

$$
\tau_{A}: \pi \mapsto \pi \wedge \mathbf{1}_{A}=\{B \cap A \mid B \in \pi, B \cap A \neq \emptyset\}
$$

where $\wedge$ is the infimum operation for the standard order. Next we define the restriction to $A$ :

$$
\rho_{A}: \pi \mapsto \pi \cap \mathcal{P}(A)=\{B \in \pi \mid B \subseteq A\}
$$

In terms of our orders, we have to see which ones are compatible with these two operations, that is: given an order $\stackrel{x}{\leq}$, does $\pi_{1} \stackrel{x}{\leq} \pi_{2}$ imply $\tau_{A}\left(\pi_{1}\right) \stackrel{x}{\leq} \tau_{A}\left(\pi_{2}\right)$ or $\rho_{A}\left(\pi_{1}\right) \stackrel{x}{\leq} \rho_{A}\left(\pi_{2}\right) ?$

We saw in [16] that the standard, merging, inclusion and inclusion-inflating orders are compatible with truncation by $A$, but the inflating, merging-inflating and building orders are not. For the restriction to $A,(11)$ indicates that the building order is compatible with it. We showed that any order included in the standard order, that is compatible with restriction to A, must be included in the inclusion order, and the inclusion order is indeed compatible with it.

We will now see that our three new orders (apportioning, apportioning-inflating and extended) are compatible with neither truncation by $A$ nor restriction to $A$.

Let $C, D, K, L, M, N$ be mutually disjoint non-empty subsets of $E$, and take

$$
\pi_{1}=\{C, D, K \cup L, M \cup N\}, \quad \pi_{2}=\{C \cup K \cup M, D \cup L \cup N\} ;
$$


then $\pi_{1} \stackrel{\text { a }}{\leq} \pi_{2}$, so $\pi_{1} \stackrel{\text { ai }}{\leq} \pi_{2}$ and $\pi_{1} \stackrel{\text { e }}{\leq} \pi_{2}$. Now let $A=E \backslash C$; thus

$$
\tau_{A}\left(\pi_{1}\right)=\{D, K \cup L, M \cup N\}, \quad \tau_{A}\left(\pi_{2}\right)=\{K \cup M, D \cup L \cup N\} ;
$$

both have the same support $D \cup K \cup L \cup M \cup N$, but the block $K \cup M$ of $\tau_{A}\left(\pi_{2}\right)$

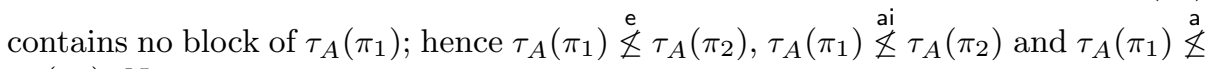
$\tau_{A}\left(\pi_{2}\right)$. Now

$$
\rho_{A}\left(\pi_{1}\right)=\{D, K \cup L, M \cup N\}, \quad \rho_{A}\left(\pi_{2}\right)=\{D \cup L \cup N\} ;
$$

here the support of $\rho_{A}\left(\pi_{2}\right)$ is strictly included in the support of $\rho_{A}\left(\pi_{1}\right)$; hence $\rho_{A}\left(\pi_{1}\right) \stackrel{\mathrm{e}}{\leq} \rho_{A}\left(\pi_{2}\right)$, so $\rho_{A}\left(\pi_{1}\right) \stackrel{\text { ai }}{\leq} \rho_{A}\left(\pi_{2}\right)$ and $\rho_{A}\left(\pi_{1}\right) \stackrel{\text { a }}{\star} \rho_{A}\left(\pi_{2}\right)$.

We indicated in the Introduction some possible applications of the apportioning, apportioning-inflating and extended orders in image filtering and segmentation. This should be experimentally tested. Such orders could also be applied to image filtering, cf. Figure 5.

The ten orders of Figure 14, that is, the identity, the six ones studied in [16] and the three new ones introduced in this paper, are all compatible with support inclusion: when $\pi_{1} \leq \pi_{2}$, we have $\operatorname{supp}\left(\pi_{1}\right) \subseteq \operatorname{supp}\left(\pi_{2}\right)$. At the end of [16] we briefly mentioned other possible orders that behave contrarily: when a partial partition grows, the support decreases or remains the same, $\pi_{1} \stackrel{x}{\leq} \pi_{2} \Rightarrow \operatorname{supp}\left(\pi_{1}\right) \supseteq \operatorname{supp}\left(\pi_{2}\right)$. One is the regional order, generated by the merging ${ }^{\mathrm{m}} \leq$ and inverse inclusion $\supseteq$ orders, that is, a partial partition grows by merging or removing blocks. (Corrigendum : we wrote that its least and greates elements are respectively $\mathbf{1}_{E}$ and $\mathbf{0}_{E}$, in fact they are $\mathbf{0}_{E}$ and $\varnothing$ ). In a future paper, we will study orders on partial partitions containing the regional order, for which the growth of a partial partition can only diminish its support and its size; such orders can be envisaged for the simplification of objects made of several parts.

\section{References}

1. Bertrand, G., Couprie, M., Cousty, J., Najman, L.: Watersheds in discrete spaces. In: L. Najman, H. Talbot (eds.) Mathematical Morphology: From Theory to Applications, chap. 9, pp. 229-261. ISTE / J. Wiley \& Sons (2010)

2. Birkhoff, G.: Lattice Theory, American Mathematical Society Colloquium Publications, vol. 25, 8th printing, 3rd edn. American Mathematical Society (1995)

3. Blyth, T.: Lattices and Ordered Algebraic Structures. Springer, London (2005)

4. Draškovičová, H.: The lattice of partitions in a set. Acta Fac. Rerum Natur. Univ. Comenian. Math. Publ. 24, 37-65 (1970)

5. Draškovičová, H.: Congruence relations on the lattice of partitions in a set. Matematický Casopis (Slovenska Akademia Vied) 21, 141-153 (1971)

6. Gomila, C.: Mise en correspondance de partitions en vue du suivi d'objets. Ph.D. thesis, Ecole des Mines de Paris (2001)

7. Grätzer, G.: General Lattice Theory. Birkhäuser, 2nd edition, Basel (2003)

8. Meyer, F., Najman, L.: Segmentation, minimum spanning tree and hierarchies. In: L. Najman, H. Talbot (eds.) Mathematical Morphology: From Theory to Applications, chap. 3, pp. 81-107. ISTE / J. Wiley \& Sons (2010)

9. Najman, L., Schmitt, M.: Geodesic saliency of watershed contours and hierarchical segmentation. IEEE Transactions on Pattern Analysis and Machine Intelligence 18(12), 1163-1173 (1996) 
10. Noyel, G., Angulo, J., Jeulin, D.: On distances, paths and connections for hyperspectral image segmentation. In: G. Banon, J. Barrera, U. Braga-Neto (eds.) Proceedings of ISMM 2007, the 8th International Symposium on Mathematical Morphology, pp. 399-410. $\mathrm{MCT} / \mathrm{INPE}(2007)$

11. Ore, O.: Theory of equivalence relations. Duke Mathematical Journal 9, 573-627 (1942)

12. Pavlidis, T.: Structural Pattern Recognition. Springer, Berlin (1980)

13. Ronse, C.: Partial partitions, partial connections and connective segmentation. Journal of Mathematical Imaging and Vision 32(2), 97-125 (2008). DOI 10.1007/s10851-008-0090-5

14. Ronse, C.: Adjunctions on the lattices of partitions and of partial partitions. Applicable Algebra in Engineering, Communication and Computing 21(5), 343-396 (2010). DOI $10.1007 / \mathrm{s} 00200-010-0129-\mathrm{x}$

15. Ronse, C.: Orders on partial partitions and maximal partitioning of sets. In: P. Soille, G. Ouzounis, M. Pesaresi (eds.) Proceedings of ISMM 2011, the 10th International Symposium on Mathematical Morphology, Lecture Notes in Computer Science, vol. 6671, pp. 49-60. Springer-Verlag, Berlin (2011)

16. Ronse, C.: Ordering partial partitions for image segmentation and filtering: merging, creating and inflating blocks. Journal of Mathematical Imaging and Vision 49(2), 202-233 (2014). DOI 10.1007/s10851-013-0455-2

17. Ronse, C., Serra, J.: Algebraic foundations of morphology. In: L. Najman, H. Talbot (eds.) Mathematical Morphology: From Theory to Applications, chap. 2, pp. 35-80. ISTE / J. Wiley \& Sons (2010)

18. Salembier, P.: Connected operators based on tree pruning strategies. In: L. Najman, H. Talbot (eds.) Mathematical Morphology: From Theory to Applications, chap. 7, pp. 179-198. ISTE / J. Wiley \& Sons (2010)

19. Salembier, P., Wilkinson, M.H.F.: Connected operators: A review of region-based morphological image processing techniques. IEEE Signal Processing Magazine 26(6), 136-157 (2009)

20. Serra, J.: Morphological segmentations of colour images. In: C. Ronse, L. Najman, E. Decencière (eds.) Mathematical Morphology: 40 Years On, Computational Imaging and Vision, vol. 30, pp. 151-176. Springer-Verlag, Dordrecht (2005)

21. Serra, J.: A lattice approach to image segmentation. Journal of Mathematical Imaging and Vision 24(1), 83-130 (2006)

22. Serra, J.: Ordre de la construction et segmentations hiérarchiques. Tech. rep., ESIEE/A2SI/IGM (2010)

23. Serra, J.: Grain building ordering. In: P. Soille, G. Ouzounis, M. Pesaresi (eds.) Proceedings of ISMM 2011, the 10th International Symposium on Mathematical Morphology, Lecture Notes in Computer Science, vol. 6671, pp. 37-48. Springer-Verlag, Berlin (2011)

24. Serra, J., Vachier, C., Meyer, F.: Levelings. In: L. Najman, H. Talbot (eds.) Mathematical Morphology: From Theory to Applications, chap. 8, pp. 199-228. ISTE / J. Wiley \& Sons (2010)

25. Soille, P.: Constrained connectivity for hierarchical image partitioning and simplification. IEEE Transactions on Pattern Analysis and Machine Intelligence 30(7), 1132-1145 (2008)

26. Velasco-Forero, S., Angulo, J., Soille, P.: Conditional toggle mappings: Principles and applications. Journal of Mathematical Imaging and Vision 48(3), 544-565 (2014). DOI $10.1007 / \mathrm{s} 10851-013-0429-4$

\section{A The bad properties of the building order}

Here we will show that the building order is not graded and does not satisfy the JordanDedekind chain condition; moreover its covering chains (of variable length) have a strange form, see Figure 15; even by restricting chains to a more orderly form, their length can still vary, see Figure 16. This is linked to the fact that such chains combine the removal of a block of variable size with repeated inflations of one block by one point, alternating variable decreases and unitary increases of the support of the partial partition.

For $\pi_{1}, \pi_{2} \in \Pi^{*}(E)$, let us write $\pi_{1} \stackrel{\mathrm{d}}{\prec} \pi_{2}$ if $\pi_{1}$ is a partition of $E$ and $\pi_{2}$ is obtained by removing a block of $\pi_{1}$ :

$$
\pi_{1} \stackrel{\mathrm{d}}{\prec} \pi_{2} \Longleftrightarrow\left[\pi_{1} \in \Pi(E) \& \pi_{1} \stackrel{\mathrm{c}}{\succ} \pi_{2}\right] .
$$


We will study the covering chains for the order $\Subset$, but also chains that follow the two steps proposed by Serra and Proposition 3: first we successively remove individual blocks, next we successively inflate the remaining blocks; for $\pi_{1} \Subset \pi_{2}$ among such chains between $\pi_{1}$ and $\pi_{2}$, a maximal one takes the form $\pi_{1} \succ^{c} \cdots \stackrel{c}{\succ} \pi \stackrel{i}{\prec} \cdots \stackrel{i}{\prec} \pi_{2}$; we will call it a normalized chain. In both cases, the length of such a chain will vary:

Proposition 14 The covering relation of the building order $\Subset i s \stackrel{\mathrm{i}}{\prec} \cup \stackrel{\mathrm{d}}{\prec}$. When $E$ is finite, $\left(\Pi^{*}(E), \Subset\right)$ is not graded and does not satisfy the Jordan-Dedekind chain condition; for $\pi_{1} \Subset$ $\pi_{2}$, in a covering chain between $\pi_{1}$ and $\pi_{2}$, the number of $d$-coverings $\stackrel{\mathrm{d}}{\prec}$ is $\left|\pi_{1}\right|-\left|\pi_{2}\right|$, but the number of $i$-coverings $\stackrel{i}{\prec}$ can vary. In a normalized chain $\pi_{1} \stackrel{c}{\succ} \cdots \stackrel{c}{\succ} \pi \stackrel{i}{\prec} \cdots \stackrel{i}{\prec} \pi_{2}$, the number of inverse c-coverings $\stackrel{c}{\succ}$ is $\left|\pi_{1}\right|-\left|\pi_{2}\right|$, but the number of $i$-coverings $\stackrel{\mathrm{i}}{\prec}$ can vary.

Proof Let $\pi_{1} \stackrel{i}{\prec} \pi_{2}$. If $\pi_{1} \Subset \pi \Subset \pi_{2}$, then (12) gives $\pi_{0} \stackrel{i}{\leq} \pi_{1} \stackrel{i}{\leq} \pi_{2}$, and as $\stackrel{i}{\prec}$ is the covering relation for $\stackrel{i}{\leq}$ (Table 1 ), we get $\pi=\pi_{1}$ or $\pi=\pi_{2}$. Hence $\pi_{2}$ covers $\pi_{1}$ for $\Subset$. Now let $\pi_{1} \stackrel{\text { d }}{\prec} \pi_{2}$; thus $\pi_{1} \in \bar{\Pi}(E)$ and there is $B \in \pi_{1}$ such that $\pi_{2}=\pi_{1} \backslash\{B\}$; then $B=E \backslash \operatorname{supp}\left(\pi_{2}\right)$. If $\pi_{1} \Subset \pi \Subset \pi_{2}$, then for every $C \in \pi_{2}$, there exist $A \in \pi$ and $D \in \pi_{1}$ such that $D \subseteq A \subseteq C$; we may not have $D=B$, so $D \in \pi_{2}$ and $D=C$, hence $A=C$. Thus $\pi_{2} \subseteq \pi$. If $\pi \neq \pi_{2}$, let $A \in \pi \backslash \pi_{2}$; thus $A$ is disjoint from $\operatorname{supp}\left(\pi_{2}\right)$, so $A \subseteq E \backslash \operatorname{supp}\left(\pi_{2}\right)=B$; now there is some $C \in \pi_{1}$ such that $C \subseteq A$, and necessarily $C=B$; thus $A=B$ and we get $\pi=\pi_{1}$. Therefore $\pi_{2}$ covers $\pi_{1}$.

Conversely, suppose that $\pi_{2}$ covers $\pi_{1}$. We obtain $\pi_{1} \supseteq \pi \stackrel{i}{\leq} \pi_{2}$. The three cases $\pi_{1} \supset$ $\pi \stackrel{i}{<} \pi_{2}, \pi_{1}=\pi \stackrel{i}{<} \pi^{\prime} \stackrel{i}{<} \pi_{2}$ and $\pi_{1} \supset \pi^{\prime} \supset \pi=\pi_{2}$ contradict the covering of $\pi_{1}$ by $\pi_{2}$, so there remain only the cases where $\pi_{1}$ covers $\pi_{2}$ for $\subseteq$ or $\pi_{2}$ covers $\pi_{1}$ for $\stackrel{\text { i }}{\leq}$, that is, $\pi_{2} \stackrel{c}{\prec} \pi_{1}$ or $\pi_{1} \stackrel{\text { i }}{\prec} \pi_{2}$ (cf. Table 1). If $\pi_{2} \stackrel{\mathrm{c}}{\prec} \pi_{1}$, there is $B \in \pi_{1}$ such that $\pi_{2}=\pi_{1} \backslash\{B\}$; now if $\pi_{1} \notin \Pi(E)$, there is some $p \in E \backslash \operatorname{supp}\left(\pi_{1}\right)$ and we get $\pi_{1}=\pi_{2} \cup\{B\} \stackrel{\mathrm{i}}{<} \pi_{2} \cup\{B \cup\{p\}\} \supset \pi_{2}$, so $\pi_{1} \Subset \pi_{2} \cup\{B \cup\{p\}\} \Subset \pi_{2}$, and $\pi_{2}$ does not cover $\pi_{1}$; hence $\pi_{1} \in \Pi(E)$ and so $\pi_{1} \stackrel{\mathrm{d}}{\prec} \pi_{2}$. We have thus shown that the covering relation is $\stackrel{i}{\prec} \cup \stackrel{d}{\prec}$.

Let $E$ be finite. Each $d$-covering $\stackrel{\mathrm{d}}{\prec}$ corresponds to the removal of one block, thus to a decrease of the number of blocks by 1 , while an $i$-covering $\stackrel{\text { i }}{\prec}$ does not change the number of blocks. Hence for $\pi_{1} \Subset \pi_{2}$, in a covering chain between $\pi_{1}$ and $\pi_{2}$, the number of $d$-coverings is $\left|\pi_{1}\right|-\left|\pi_{2}\right|$. Each inverse $c$-coverings $\succ^{c}$ corresponds to the removal of one block, thus to a decrease of the number of blocks by 1 , while an $i$-covering $\stackrel{i}{\prec}$ does not change the number of blocks. Hence for $\pi_{1} \Subset \pi_{2}$, a normalized chain has exactly $\left|\pi_{1}\right|-\left|\pi_{2}\right|$ inverse $c$-coverings $\underbrace{c}$.

However in both covering chains and normalized chains, the number of $i$-coverings can vary. Take two disjoint non-void subsets $A, B$ of $E$, where $|A|<|B|$, let $\pi_{1}=\{A, B\}$ and $\pi_{2}=\{A \cup B\}$, thus $\pi_{1} \Subset \pi_{2}$. A covering chain between $\pi_{1}$ and $\pi_{2}$ is obtained by first adding successively to $B$ the points of $E \backslash(A \cup B)$ to reach $\{A, E \backslash A\}$ after $|E|-|A|-|B|$ steps, then removing the block $E \backslash A$ to get $\{A\}$ in 1 step, and finally adding successively to $A$ the points of $B$ in $|B|$ steps; this covering chain has length $1+|E|-|A|$. Exchanging the roles of $A$ and $B$, we get another covering chain of length $1+|E|-|B|$. See Figure 15. Both chains have $1 d$-covering, but the number of $i$-coverings is respectively $|E|-|A|$ and $|E|-|B|$. Hence $\left(\Pi^{*}(E), \Subset\right)$ does not satisfy the Jordan-Dedekind chain condition, therefore it is not graded. Now we obtain a normalized chain between $\pi_{1}$ and $\pi_{2}$ by first removing the block $B$ in 1 inverse $c$-covering, then adding successively to $A$ the points of $B$ in $|B| i$-coverings; this normalized chain has length $1+|B|$. Exchanging the roles of $A$ and $B$, we get another chain of length $1+|A|$, with 1 inverse $c$-covering and $|A| i$-coverings. See Figure 16 .

The fact that $\left(\Pi^{*}(E), \Subset\right)$ does not satisfy the Jordan-Dedekind chain condition is a serious defect, it means that the progressive construction of a segmentation by iterating elementary steps does not have a fixed complexity (unless we give to $i$-coverings a zero cost). Moreover, 


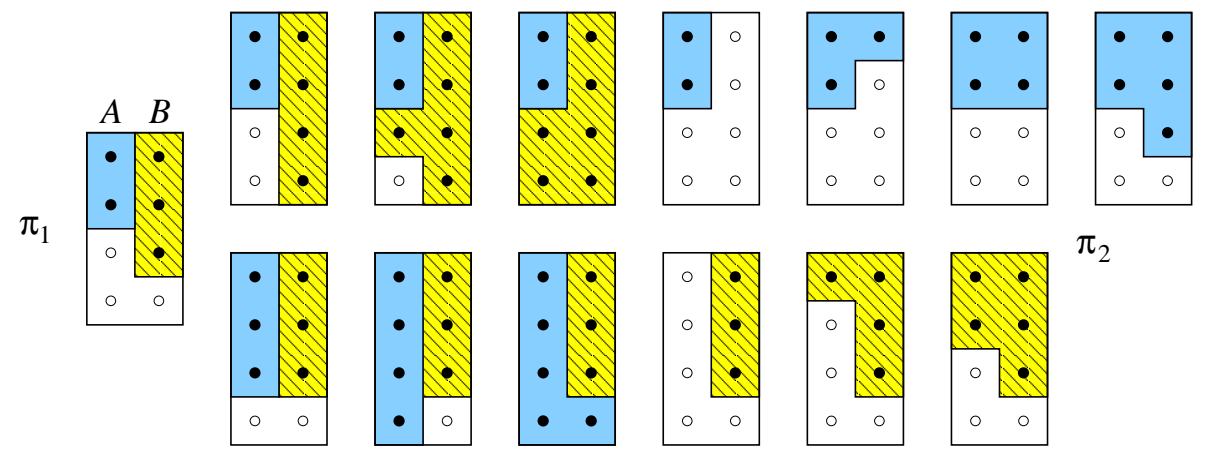

Fig. 15 Here $|E|=8,|A|=2,|B|=3, \pi_{1}=\{A, B\}$ and $\pi_{2}=\{A \cup B\}$. Top line: the covering chain $\pi_{1}=\{A, B\} \stackrel{\text { i }}{\prec} \ldots \stackrel{\text { i }}{\prec}\{A, E \backslash A\} \stackrel{\text { d }}{\prec}\{A\} \stackrel{\text { i }}{\prec} \ldots \stackrel{\text { i }}{\prec}\{A \cup B\}=\pi_{2}$ has length $3+1+3=7$. Bottom line: the covering chain $\pi_{1}=\{A, B\} \stackrel{\mathrm{i}}{\prec} \ldots \stackrel{\mathrm{i}}{\prec}\{E \backslash B, B\} \stackrel{\mathrm{d}}{\prec}\{B\} \stackrel{\mathrm{i}}{\prec} \ldots \stackrel{\mathrm{i}}{\prec}\{A \cup B\}=\pi_{2}$ has length $3+1+2=6$.

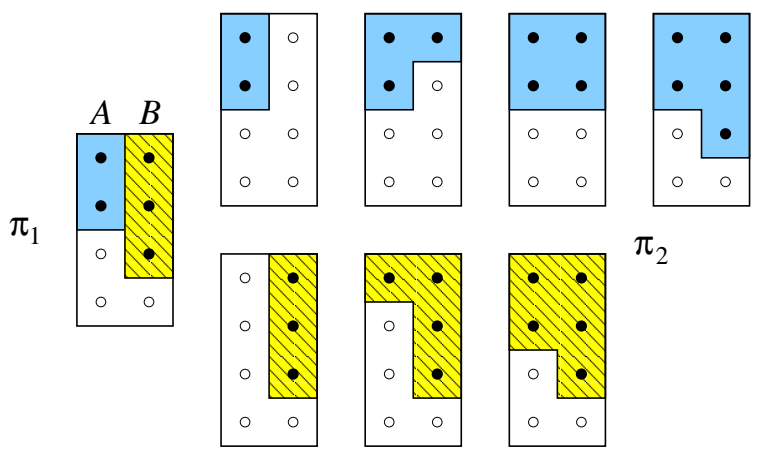

Fig. 16 Take $E, A, B, \pi_{1}$ and $\pi_{2}$ as in Figure 15. Top line: the chain $\pi_{1}=\{A, B\} \stackrel{c}{\succ}\{A\} \stackrel{\text { i }}{\prec}$ $\ldots \stackrel{i}{\prec}\{A \cup B\}=\pi_{2}$ has length $1+3=4$. Bottom line: the chain $\pi_{1}=\{A, B\} \stackrel{c}{\succ}\{B\} \stackrel{i}{\prec} \ldots \stackrel{i}{\prec}$ $\{A \cup B\}=\pi_{2}$ has length $1+2=3$.

the form taken by covering chains is counterintuitive, as seen in Figure 15: in order to remove a block, we need first to inflate it until it fills the background.

The problem in Figures 15 and 16 is that the chains combine inflating a block with one point, which increases the size of the support by exactly one, with the removal of an arbitrary block, which decreases the size of the support by a variable amount. Thus the number of block inflations necessary to compensate for the block removals will depend on the size of removed blocks.

We conclude that the building order is a very useful mathematical tool for the design of meaningful orders on partial partitions; indeed it was used in [16] for the definition of the inflating and merging-inflating orders, then in the present paper for the definition of the apportioning, apportioning-inflating and extended orders. Its direct use for comparing partial partitions can be appropriate only if both operations generating it, namely removing and inflating blocks, correspond to an improvement of a partial partition. However, this order is not graded and has bizarre covering chains of variable length (cf. Figure 15), while normalized chains have a more orderly form, but their length still varies (cf. Figure 16). These defects indicate that this order should not be used for the analysis of a gradual growth of partial partitions. 